\title{
Global Star Formation Rates and Dust Emission Over the Galaxy Interaction Sequence
}

\section{Citation}

Lanz, Lauranne, Andreas Zezas, Nicola Brassington, Howard A. Smith, Matthew L. N. Ashby, Elisabete da Cunha, Giovanni G. Fazio, Christopher C. Hayward, Lars Hernquist, and Patrik Jonsson. 2013. "GLOBAL STAR FORMATION RATES AND DUST EMISSION OVER THE GALAXY INTERACTION SEQUENCE." The Astrophysical Journal 768 (1): 90. https:// doi.org/10.1088/0004-637x/768/1/90.

\section{Permanent link}

http://nrs.harvard.edu/urn-3:HUL.InstRepos:41381637

\section{Terms of Use}

This article was downloaded from Harvard University's DASH repository, and is made available under the terms and conditions applicable to Open Access Policy Articles, as set forth at http:// nrs.harvard.edu/urn-3:HUL.InstRepos:dash.current.terms-of-use\#OAP

\section{Share Your Story}

The Harvard community has made this article openly available.

Please share how this access benefits you. Submit a story.

\section{Accessibility}


Draft VERSiOn June 20, 2018

Preprint typeset using $\mathrm{IAT}_{\mathrm{E}} \mathrm{X}$ style emulateapj v. 5/2/11

\title{
GLOBAL STAR FORMATION RATES AND DUST EMISSION OVER THE GALAXY INTERACTION SEQUENCE
}

\author{
Lauranne Lanz ${ }^{1}$, Andreas Zezas ${ }^{1,2,3}$, Nicola Brassington ${ }^{4}$, Howard A. Smith ${ }^{1}$, Matthew L. N. Ashby ${ }^{1}$, Elisabete \\ da Cunha ${ }^{5}$, Giovanni G. Fazio ${ }^{1}$, Christopher C. Hayward ${ }^{6}$, Lars Hernquist ${ }^{1}$, Patrik Jonsson ${ }^{7}$ \\ Draft version June 20, 2018
}

\begin{abstract}
We measured and modeled the spectral energy distributions (SEDs) in 28 bands from the ultraviolet to the far-infrared (FIR) for 31 interacting galaxies in 14 systems. The sample is drawn from the Spitzer Interacting Galaxy Survey, which probes a range of galaxy interaction parameters at multiple wavelengths with an emphasis on the infrared bands. The subset presented in this paper consists of all galaxies for which FIR Herschel SPIRE observations are publicly available. Our SEDs combine the Herschel photometry with multi-wavelength data from Spitzer, GALEX, Swift UVOT, and 2MASS. While the shapes of the SEDs are broadly similar across our sample, strongly interacting galaxies typically have more mid-infrared emission relative to their near-infrared and FIR emission than weakly or moderately interacting galaxies. We modeled the full SEDs to derive host galaxy star formation rates (SFR), specific star formation rates (sSFR), stellar masses, dust temperatures, dust luminosities, and dust masses. We find increases in the dust luminosity and mass, SFR, and cold (15-25 K) dust temperature as the interaction progresses from moderately to strongly interacting and between noninteracting and strongly interacting galaxies. We also find increases in the SFR between weakly and strongly interacting galaxies. In contrast, the sSFR remains unchanged across all the interaction stages. The ultraviolet photometry is crucial for constraining the age of the stellar population and the SFR, while dust mass is primarily determined by SPIRE photometry. The SFR derived from the SED modeling agrees well with rates estimated by proportionality relations that depend on infrared emission.

Subject headings: infrared: galaxies, galaxies: interactions, galaxies: photometry, galaxies: star formation, ultraviolet: galaxies
\end{abstract}

\section{INTRODUCTION}

Galaxy evolution is believed to be heavily influenced by interactions between galaxies, both for local systems and for distant objects at earlier cosmological times. In the canonical view, interactions between galaxies have three primary observable effects. In the most dramatic cases, interactions stimulate star formation in a burst of activity that is presumed to power the high infrared (IR) luminosities typically seen in such systems. Many local ultra-luminous IR galaxies ( $\mathrm{L} \geq 10^{12} \mathrm{~L}_{\odot}$; ULIRGs) and luminous IR galaxies $\left(10^{11} \mathrm{~L}_{\odot} \leq \mathrm{L} \leq 10^{12} \mathrm{~L}_{\odot}\right.$; LIRGs $)$ show evidence of galaxy interactions (e.g., Veilleux et al. 2002). Similarly, their high-redshift counterparts, sub-millimeter galaxies, first detected by SCUBA and now studied extensively by the Spectral and Photometric Imaging Receiver (SPIRE) on the Herschel Space Obser-

\footnotetext{
${ }^{1}$ Harvard-Smithsonian Center for Astrophysics, 60 Garden St., Cambridge, MA 02138, USA; llanz@head.cfa.harvard.edu 2 University of Crete, Physics Department \& Institute of Theoretical \& Computational Physics, 71003 Heraklion, Crete, Greece

${ }^{3}$ Foundation for Research and Technology-Hellas, 71110 Heraklion, Crete, Greece

${ }^{4}$ School of Physics, Astronomy and Mathematics, University of Hertfordshire, College Lane, Hatfield, AL10 9AB, UK

${ }^{5}$ Max Planck Institute for Astronomy (MPIA), Königstuhl 17, 69117, Heidelberg, Germany

${ }^{6}$ Heidelberger Institut für Theoretische Studien, SchlossWolfsbrunnenweg 35, 69118, Heidelberg, Germany

7 Space Exploration Technologies, 1 Rocket Road, Hawthorne, $\mathrm{CA}, 90250$
}

vatory ${ }^{8}$, are thought to be predominantly mergers (e.g., Blain et al. 1999), although the relative contribution of mergers of different stages to their numbers is still an open question (e.g., Hayward et al. 2012a, 2012b).

The second effect is that interactions significantly affect the subsequent evolution of galaxies, which may lead to significant changes in their morphology. Disturbed galaxies have long been associated with mergers (e.g., Toomre \& Toomre 1972). Numerical simulations of interactions (e.g., Hopkins et al. 2006; Hopkins 2012; Mihos \& Hernquist 1994, 1996; Barnes 1992; Barnes \& Hernquist 1996; Sanders 1999) show a variety of morphological distortions as well as variable amounts of star formation. The simulations also demonstrate the complexity of the problem: the degree of induced activity and distortion varies greatly with the parameters of the encounter, the phase of the interactions, the molecular gas content ("wetness"), and the mass of the progenitor galaxies among many other properties.

Third, the canonical picture, as seen in many simulations (e.g., Di Matteo et al. 2005, Springel et al. 2005), involves merger-driven gas inflow to the central regions, resulting in heightened activity of the central supermassive black hole as well as starburst activity due to the increased central gas density and possibly turbulence. The process in principle converts a low-luminosity nucleus into an active galactic nucleus (AGN) but one whose lu-

\footnotetext{
${ }^{8}$ Herschel is an ESA space observatory with science instruments provided by European-led Principal Investigator consortia and with important participation from NASA.
} 
minosity might range widely depending on the stage of the interaction. Indeed, observations of merging galaxies over the years have tended to provide evidence supporting the conclusion that, at least on a statistical level, interactions trigger an enhancement in the formation of stars as well as nuclear activity. However, the recent literature includes works that argue both for and against a strong connection between nuclear activity and mergers (e.g., Li et al. 2008, Kocevski et al. 2012, Ellison et al. 2011, Scudder et al. 2012, Silverman et al. 2011).

Therefore, despite many previous studies (e.g., Dahari 1985, Sanders et al. 1988, Kewley et al. 2001, Lambas et al. 2003), both observational and through simulations, our understanding of the evolution of the physical activity during the course of a galaxy-galaxy interaction remains incomplete. In the past decade, two new developments have dramatically changed our understanding of star formation and accretion activity around galactic nuclei, which are the two dominant processes at work in controlling the observed emission. The first is the success of space missions, in particular, the Spitzer Space Telescope $^{9}$ (Werner et al. 2004) and the Herschel Space Observatory (Pilbratt et al. 2010) in the IR, as well as the Galaxy Evolution Explorer ${ }^{10}$ (GALEX; Martin et al. 2005) and Swift (Gehrels et al. 2004) in the ultraviolet (UV), providing photometry across the complete spectral range from UV to millimeter wavelengths. Most importantly, detailed imaging and high sensitivity photometry now available at the critical far-infrared (FIR) emission peak resulting from warm, luminous dust heated by starbursts provides crucial information regarding dust heating and embedded star formation. The combination of UV and IR observations is essential to obtain a complete census of recent and ongoing star formation by capturing both the unobscured and obscured emission from young stars.

The second development has been the success of computational codes. We have new tools for the derivation of galaxy properties including masses, star formation rates, and interstellar medium (ISM) parameters from global fits, which allow self-consistent measurements of critical parameters combining stellar evolution models (e.g., Bruzual \& Charlot 2003) with radiative transfer through a dusty ISM (e.g., Charlot \& Fall 2000). A second set of tools uses sophisticated hydrodynamic computational codes to simulate interactions (e.g., GADGET - Springel 2005 ), while simultaneously new radiative transfer models can compute the predicted emission from these evolving interacting systems (e.g., SUNRISE - Jonsson 2006).

It is important to recognize that observational biases can be significant. Due to the long timescales of an interaction (typically $10^{8-9}$ years), observers rely on studies of a range of interacting systems to reconstruct a likely sequence of events. Moreover, determining the exact phase of any particular observed interaction from its morphology is uncertain at best, because the appearance of a system at a given interaction phase also depends on the specific geometry of the encounter, the masses of the galaxies, metallicity, molecular gas content, and

\footnotetext{
9 Spitzer is operated by the Jet Propulsion Laboratory, California Institute of Technology under a contract with NASA.

$10 G A L E X$ is operated for NASA by the California Institute of Technology under NASA contract NAS5-98034.
}

(not least) previous interaction histories (e.g., Di Matteo et al. 2007). Systems are ordered into an evolutionary sequence using intuition provided by simulations and physical models, which are themselves based on observations of particular systems. Selection criteria, however, can introduce a bias for more luminous, morphologically disturbed systems and, hence, towards the most active phases of interactions. Therefore, a selection criterion not associated with either morphological disturbance or degree of activity is critical for obtaining a sample containing systems throughout the full interaction sequence.

We have undertaken a program to take advantage of all these developments: full, multi-wavelength datasets of an interacting galaxy sample selected with few biases; hydrodynamic simulations; and radiative transfer modeling, in a systematic effort to better understand systems across a range of interaction stages and to iterate refinements to the various modeling and radiative transfer codes. We have chosen a representative sample of objects spanning the interaction sequence, obtained their full spectral energy distributions (SEDs), and are comparing the results against a variety of models - based on both templates/stellar evolution/radiative transfer and on diagnostic features.

This first paper of the project presents results and conclusions for a sample of thirty-one interacting galaxies in fourteen systems for which there are currently complete multi-wavelength data that can be used to study the variations in their star formation and dust heating. This paper is organized as follows. We describe the full Spitzer Interacting Galaxy Survey (SIGS) sample in Section 2 and the classification of each of the sources in the interaction sequence. Section 3 describes the wide range of observational photometry used to construct the SEDs. It also describes the issues associated with obtaining reliable photometry from the diverse datasets. In Section 4, we model the SEDs of these objects. Section 5 discusses the variations seen across the interaction sequence and constraints imposed by photometry from different instruments and compares star formation rates derived using the entire SED to those from relations depending on one or two wavelengths. In Section 6, we summarize our results.

\section{THE SPITZER INTERACTING GALAXY SURVEY (SIGS) SAMPLE}

\subsection{Sample Description}

The SIGS sample was designed to span the full range of galaxy interaction parameters by using a sample selected strictly on the basis of interaction probability rather than morphology, activity, luminosity, or other derivative indicators. The catalog includes interactions of all types, not just those that give rise to obvious morphological peculiarities and/or nuclear/starburst activity, thus minimizing morphological biases so we can address the relationships between interactions and activity. A selection criterion not dependent on visible signs of tidal interactions is important because of the dependence of the response of interacting galaxies on the relative inclinations of disks (e.g., Toomre \& Toomre 1972; D'Onghia et al. 2010) and the uncertain distribution of dark matter around the galaxies (e.g., Dubinski et al. 1996, 1999). The SIGS sample was based on the Keel-Kennicutt visibly selected catalog of interacting spiral galaxies (Keel 
TABLE 1

SAMPLE DESCRIPTION

\begin{tabular}{|c|c|c|c|c|c|c|c|c|}
\hline $\begin{array}{l}\text { Group } \\
(1)\end{array}$ & $\begin{array}{c}\text { Galaxy } \\
(2)\end{array}$ & $\begin{array}{l}\text { R.A. } \\
\text { (J2000) } \\
(3)\end{array}$ & $\begin{array}{l}\text { Decl. } \\
(\mathrm{J} 2000) \\
(4)\end{array}$ & $\begin{array}{c}\text { Distance } \\
(\mathrm{Mpc}) \\
(5)\end{array}$ & $\begin{array}{l}\text { Interaction } \\
\text { Stage } \\
(6)\end{array}$ & $\begin{array}{l}\text { Size } \\
(7)\end{array}$ & $\begin{array}{l}\text { rture } \\
\text { Angle } \\
(8)\end{array}$ & $\begin{array}{c}\text { From } \\
(9)\end{array}$ \\
\hline \multirow[t]{4}{*}{1} & NGC2976 & 094716.3 & +675452.0 & 3.75 & $2.0 \pm 0.0$ & $3 ! 57 \times 1 ! 77$ & 5198 & $3.6 \mu \mathrm{m}$ \\
\hline & NGC3031 & 095533.2 & +690357.9 & 3.77 & $2.0 \pm 0.4$ & $10 ! 11 \times 5 ! 82$ & 64.0 & $3.6 \mu \mathrm{m}$ \\
\hline & NGC3034 & 095552.2 & +694047.8 & 3.89 & $2.0 \pm 0.4$ & $2 ! 87 \times 11^{\prime} 07$ & 336.4 & $3.6 \mu \mathrm{m}$ \\
\hline & NGC3077 & 100319.8 & +684401.5 & 3.93 & $2.0 \pm 0.5$ & $2 ! 12 \times 1 ! 62$ & $318: 5$ & $3.6 \mu \mathrm{m}$ \\
\hline \multirow[t]{3}{*}{2} & NGC3185 & 101738.7 & +214116.2 & 22.6 & $2.0 \pm 0.5$ & $1 ! 84 \times 0 ! 99$ & $41 \% 9$ & NUV \\
\hline & NGC3187 & 101748.4 & +215230.9 & 26.1 & $3.0 \pm 0.5$ & $2 ! 25 \times 1 ! 04$ & 338.7 & NUV \\
\hline & NGC3190 & 101805.7 & +214957.0 & 22.5 & $3.0 \pm 0.5$ & $2 ! 14 \times 0 ! 97$ & $28^{\circ} .4$ & $3.6 \mu \mathrm{m}$ \\
\hline \multirow[t]{2}{*}{3} & NGC3226 & 102327.0 & +195353.2 & 23.3 & $4.0 \pm 0.5$ & $1^{\prime} 29 \times 1^{\prime} .00$ & 302.5 & $3.6 \mu \mathrm{m}$ \\
\hline & NGC3227 & 102330.5 & +195155.1 & 20.6 & $4.0 \pm 0.5$ & $1 ! 89 \times 1 ! 03$ & $60^{\circ} 4$ & $3.6 \mu \mathrm{m}$ \\
\hline \multirow[t]{2}{*}{4} & NGC3395 & 104950.0 & +325855.2 & 27.7 & $4.0 \pm 0.5$ & $1 ! 46 \times 0 ! 89$ & 278.9 & $3.6 \mu \mathrm{m}$ \\
\hline & NGC3396 & 104955.2 & +325925.7 & 27.7 & $4.0 \pm 0.5$ & $1 ! 38 \times 00^{\prime} 60$ & $9: 6$ & $3.6 \mu \mathrm{m}$ \\
\hline \multirow[t]{2}{*}{5} & NGC3424 & 105146.9 & +325404.1 & 26.1 & $2.0 \pm 0.4$ & $1 ! 81 \times 00^{\prime} 59$ & 17.4 & NUV \\
\hline & NGC3430 & 105211.5 & +325705.0 & 26.7 & $2.0 \pm 0.4$ & $2 ! 69 \times 1 ! 46$ & 301.3 & NUV \\
\hline \multirow[t]{2}{*}{6} & NGC3448 & 105438.7 & +541821.0 & 24.4 & $3.0 \pm 0.0$ & $1^{\prime} 57 \times 0.59$ & 338.6 & $3.6 \mu \mathrm{m}$ \\
\hline & UGC6016 & 105413.4 & +541715.5 & $27.2^{*}$ & $3.0 \pm 0.0$ & $1 ! 28 \times 0 ! 67$ & 329.3 & $3.6 \mu \mathrm{m}$ \\
\hline 7 & NGC3690/IC694 & 112831.2 & +583346.7 & $48.1^{*}$ & $4.0 \pm 0.4$ & $1.20 \times 0 ! 93$ & 40.6 & $3.6 \mu \mathrm{m}$ \\
\hline \multirow[t]{2}{*}{8} & NGC3786 & 113942.5 & +315434.2 & 41.7 & $3.0 \pm 0.5$ & $11^{\prime} 04 \times 00^{\prime} 57$ & 340.7 & $3.6 \mu \mathrm{m}$ \\
\hline & NGC3788 & 113944.6 & +315554.3 & 36.5 & $3.0 \pm 0.5$ & $1 ! 32 \times 0 ! 41$ & 84.8 & $3.6 \mu \mathrm{m}$ \\
\hline 9 & NGC4038/4039 & 120153.9 & -185234.8 & 25.4 & $4.0 \pm 0.0$ & $3^{\prime} 00 \times 2 ! 33$ & 304.3 & $3.6 \mu \mathrm{m}$ \\
\hline \multirow[t]{2}{*}{10} & NGC4618 & 124132.8 & +410844.4 & 7.28 & $3.0 \pm 0.5$ & $2^{\prime} 69 \times 2^{\prime} \cdot 08$ & $284^{\circ} 1$ & $3.6 \mu \mathrm{m}$ \\
\hline & NGC4625 & 124152.6 & +411620.6 & 8.20 & $3.0 \pm 0.5$ & $1 ! .88 \times 1 ! 49$ & 296.5 & NUV \\
\hline \multirow[t]{2}{*}{11} & NGC4647 & 124332.6 & $\begin{array}{r}+113453.9\end{array}$ & 16.8 & $3.0 \pm 0.5$ & $1 ! 53 \times 1 ! 24$ & 18.6 & NUV \\
\hline & NGC4649 & 124340.0 & +113309.8 & 17.3 & $3.0 \pm 0.5$ & $1 ! 81 \times 1 ! 33$ & 34.2 & $3.6 \mu \mathrm{m}$ \\
\hline \multirow[t]{2}{*}{12} & M51A & 132954.1 & +471141.2 & 7.69 & $3.0 \pm 0.5$ & $6 ! .86 \times 4 ! .42$ & 293.5 & NUV \\
\hline & M51B & 132959.7 & +471558.5 & 7.66 & $3.0 \pm 0.5$ & $2 ! 68 \times 1^{\prime} .95$ & 18.3 & $3.6 \mu \mathrm{m}$ \\
\hline \multirow[t]{2}{*}{13} & NGC5394 & 135833.7 & $\begin{array}{r}+372714.4 \\
\end{array}$ & $56.4^{*}$ & $4.0 \pm 0.5$ & $0 ! 89 \times 00^{\prime} 50$ & 84.2 & NUV \\
\hline & NGC5395 & 135837.6 & +372541.2 & $56.4^{*}$ & $4.0 \pm 0.5$ & $2 ! 88 \times 1 ! 08$ & $87: 9$ & NUV \\
\hline \multirow[t]{2}{*}{14} & M101 & 140309.8 & +542037.3 & 6.70 & $3.0 \pm 0.5$ & $10^{\prime} .00 \times 88^{\prime} .53$ & $156^{\circ} 4$ & $3.6 \mu \mathrm{m}$ \\
\hline & NGC5474 & 140501.2 & +533911.6 & 5.94 & $3.0 \pm 0.5$ & $2 ! 53 \times 2 ! 24$ & 290.2 & $3.6 \mu \mathrm{m}$ \\
\hline
\end{tabular}

Note. - Distance moduli were obtained from Tully et al. (2008), Tully (1994), and the Extra-galactic Distance Database. The distances in column (5) marked with * did not have distance moduli and were calculated based on heliocentric velocities, corrected per Mould et al. (2000) and assuming $H_{0}=72 \mathrm{~km} \mathrm{~s}^{-1} \mathrm{Mpc}^{-1}$. The determination of interaction stage is described in Section 2.2. In column (6) we give the median and standard deviation of the classifications by the co-authors. The parameters of the elliptical apertures are given in columns ( 7 ) and (8) and we note whether it was determined on the GALEX NUV or IRAC $3.6 \mu \mathrm{m}$ image. The angle is given degrees north of west.

et al. 1985, hereafter K85), which selected galaxies based on the local density of nearby neighbors and consists of bright spiral galaxies having neighbors with typical projected separations of 4-5 effective radii. A criterion based on the relative recessional velocities $|\Delta \mathrm{v}|<600 \mathrm{~km} \mathrm{~s}^{-1}$ was imposed to exclude non-associated, projected pairs.

In order to resolve structures on scales of a few hundred pc, we limited the original sample to sources closer than $\mathrm{cz}<4000 \mathrm{~km} \mathrm{~s}^{-1}$. To investigate the effects of tidal interaction, we added a complementary set to the prime sample: the K85 "Arp Sample" with the same maximum distance as the Keel-Kennicutt complete sample. This set is based on the Arp catalog of peculiar galaxies from which K85 selected all objects showing evidence of tidal interaction not strong enough to disrupt the galactic disks (i.e., it does not bias against late stage mergers). Although K85 excluded some fainter members of the interacting groups (their selection criteria required a B band magnitude of $\left.B_{T} \leq 13.0\right)$, we include them in order to obtain a complete picture of the activity in the different interacting systems.

The total SIGS sample consists of 103 individual interacting galaxies in 48 systems. The combined galaxies span the range of interaction types, luminosities, and galaxy types. SIGS is comprised primarily of spiralspiral interactions, with some spiral-elliptical and spiralirregular interactions. Its set of systems contains both major and minor mergers, ranging from systems likely to be in first approach (e.g. NGC 3424/NGC 3430) through close passages (e.g. M51) to final collision (NGC 3690/IC 694), and span a luminosity range from $1.3 \times 10^{10}-5.1 \times 10^{14} \mathrm{~L}_{\odot}$. From this complete sample, which has a sufficiently large number of objects to allow us to study statistically the activity in interacting galaxies across a wide range of encounter parameters, we will be able to study the increase of star formation and AGN activity in interacting disk galaxies. As discussed in section 1, while there have been a significant number of studies probing star formation rate (SFR) enhancement and nuclear activity, the importance of the different interaction parameters in triggering these events is not well understood. The SIGS sample provides us with the opportunity to observe a large range of galaxies, including very early interaction stages. The level and distribution of star-formation in such early stage interactions has not been systematically studied before, therefore our sample will allow us to identify the initial increase in SFR caused by the interaction, as well as identify where this enhancement is located in the galaxies (i.e. in the central region of the galaxy, along the disk, or within tidal features). Additionally, the size of our sample also provides us with the ability to probe these enhancements for all systems as a function of different interaction parameters, such as galaxy mass, mass ratio and gas content. A detailed description of the SIGS sample along with the analysis of the Spitzer data and a presentation of the images and the 
photometric results is given in Brassington et al. (2013, in preparation).

There are currently fourteen interacting systems from the SIGS set which have publicly available observations by all the facilities: Herschel (SPIRE and partial coverage with the Photodetector Array Camera and Spectrometer (PACS)), Spitzer, 2 Micron All Sky Survey (2MASS), and either GALEX or Swift, enabling us to model their emission from far-UV (FUV) to FIR in 28 filters when ancillary archival measurements are added. Not all galaxies have photometric data in all filters; we used as many photometric data as available, generally 15-25. These galaxies comprise the sample we examine in this paper and were selected from the SIGS sample on the basis of available SPIRE observations. They are listed in Table 1 along with key parameters.

\subsection{Estimating the Interaction Phase}

Toomre \& Toomre (1972) were the first to systematically model and describe the morphological characteristics of interacting galaxies. Using simple simulations, they showed that tails and bridges could result from tidal forces and reconstructed the orbits that could produce the tidal features seen in some of the best known interacting systems including M51, the Mice (NGC 4676), and the Antennae (NGC 4038/4039). Their work also highlighted the close connection between observations and modeling: our classification of the interaction stages in our sample is based on theoretical descriptions of how such interactions are expected to proceed.

As Rich et al. (2012) have shown, projected distance alone is an unreliable indicator of interaction stage. We therefore used the Dopita et al. (2002) five-stage scheme to classify the interaction stage of our galaxies. By construction, our sample does not include any Stage 1 galaxies (isolated, non-interacting galaxies). Stage 2 galaxies are described as weakly interacting systems, which are close on the sky, but show minimal morphological distortion. These systems could be either before or after the first passage. Stage 3 galaxies, which we call moderately interacting, show stronger signs of morphological distortion and often tidal tails. Depending on the geometry of the encounter, these systems could be in the midst of the first or a subsequent passage. Stage 4 (strongly interacting) galaxies show strong signs of disturbance and are therefore in more evolved stage of interaction. Our sample falls into these three categories. While the SIGS sample has a Stage 5 (coalescence/post-merger systems), the sample presented in this paper does not. The SIGS sample is roughly equally divided between Stages 2-4, while the sample presented in this paper has 7,14 , and 7.

This classification method is clearly a statistical scheme in the sense that, for each individual galaxy, the classification stage does not translate directly to an interaction phase. However, since the scheme is based on morphological appearance of galaxies, it provides a direct picture of the effect of the interaction on the distributions of the stellar component of the galaxies and their star formation activity. The classification was carried out independently for each galaxy in the SIGS sample by six collaborators on the basis of appearance alone in Digitized Sky Survey (DSS) images. Stage 2 galaxies show little morphological distortion, while Stage 4 galaxies are strongly distorted. Stage 3 galaxies show some distortion in the form of tidal features, although their disks remain undisturbed. Visible DSS images are best suited for this purpose, since they trace on-going star formation as well as older stellar populations in a single image. In Appendix A, we show representative examples of the galaxies in Stages 2-4. Galaxy groups in which classifications differed by more than one stage were re-examined; the median of the classifications is used for each galaxy. Table 1 lists the interaction stage for all of the galaxies in our sample.

\subsection{Comparison Non-Interacting Sample}

As a comparison sample of non-interacting galaxies, we used a subset of the "normal" galaxy sample of Smith et al. (2007). Smith et al. (2007) identified 42 galaxies from the Spitzer Infrared Nearby Galaxies Survey (SINGS; Kennicutt et al. 2003; Dale et al. 2005) of which 26 were spirals, which had not been subject to strong distortions. We were more conservative in our definition of non-interacting, by removing galaxies associated with clusters or radial-velocity groups, and we removed the three that were not observed with SPIRE as part of the Key Insights on Nearby Galaxy: a Far Infrared Survey with Herschel (KINGFISH; Kennicutt et al. 2011). Our comparison sample is comprised of 15 galaxies: NGC 925, NGC 1291, NGC 2841, NGC 3049, NGC3184, NGC 3521, NGC 3621, NGC 3938, NGC 4236, NGC 4559, NGC 4594, NGC 4736, NGC 4826, NGC 5055, and NGC 6946. We used the distances provided in Smith et al. (2007) and the UV-MIR photometry given in Dale et al. 2007) and the FIR photometry given in Dale et al. (2012).

\section{OBSERVATIONS AND DATA REDUCTION}

The sample presented here has a complete set of nearinfrared (NIR) to FIR photometry observed by 2MASS, Spitzer, and Herschel respectively, as well as near-UV (NUV) and FUV photometry observed primarily by GALEX and completed by the Ultraviolet/Optical Telescope (UVOT) on Swift. In the next sections, we describe the observations and their reduction. The observations were supplemented with mid-infrared (MIR) to FIR fluxes measured by the Infrared Astronomical Satellite $(I R A S)$, the $70 \mu \mathrm{m}$ and $160 \mu \mathrm{m}$ Multiband Imaging Photometer (MIPS) detectors on Spitzer, and UBV fluxes from the Third Reference Catalog (RC3; de Vaucouleurs et al. 1991) where available in the literature through the NASA Extragalactic Database (NED). The MIPS $24 \mu \mathrm{m}$ fluxes from these sources typically agree within the uncertainties with those we measure. Figures 1-6 show from left to right the GALEX, 2MASS, Spitzer Infrared Array Camera (IRAC), and Herschel observations of each galaxy. Some galaxies (e.g. NGC 3031 and M51A) have similar morphology from UV to FIR. In contrast, others have distinct morphological differences between the UV and IR, such as the FIR bright spots of NGC 2976 or the extended UV disk of NGC 3430. Appendix $\mathrm{C}$ contains notes on the individual galaxies.

\subsection{Galaxy Distances}

All of the galaxies in our sample are nearby (within 60 $\mathrm{Mpc}$ ) and can therefore have peculiar velocities that contribute significantly to their recessional velocities. Tully 


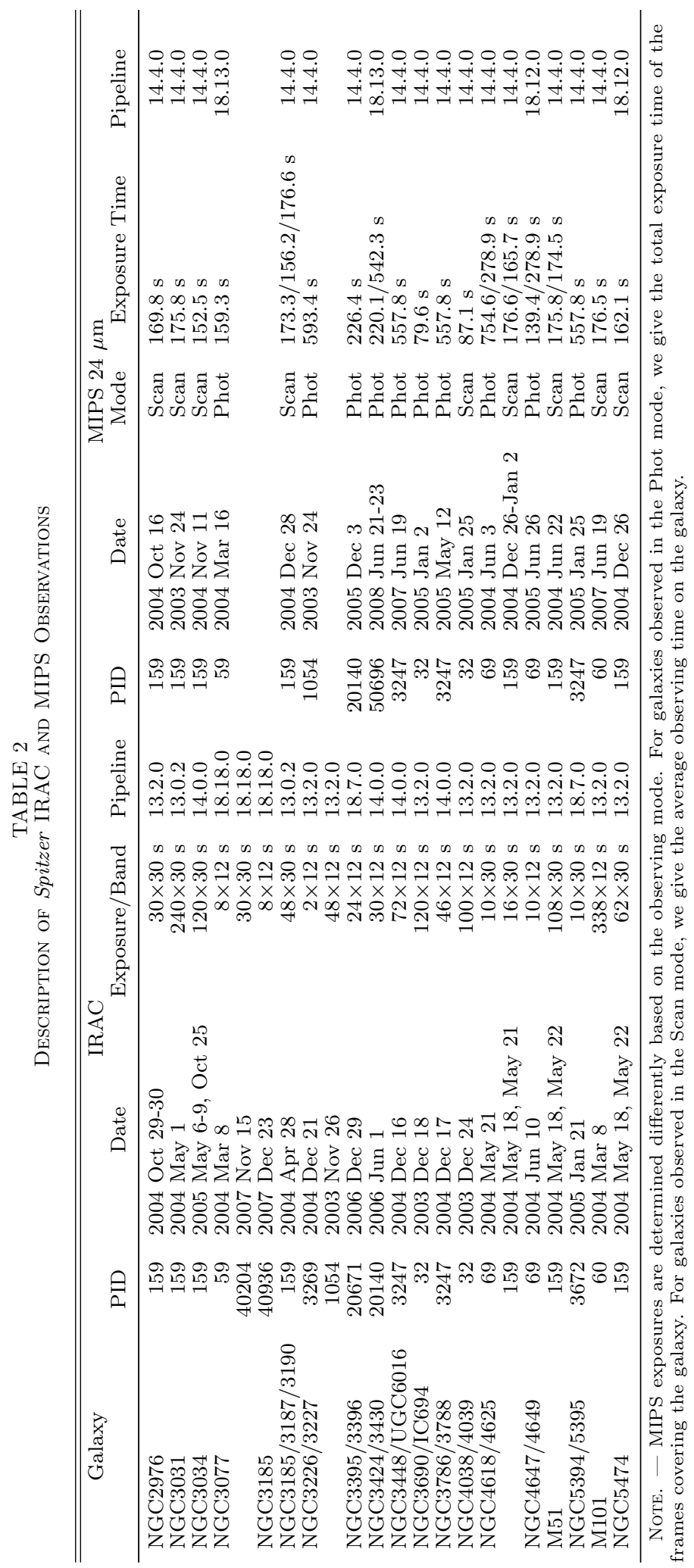


TABLE 3

Description of Herschel ObSERVATIONs

\begin{tabular}{|c|c|c|c|c|c|c|}
\hline Galaxy & Instrument & ObsID & Date & Obs. Mode & Exposure (s) & PACS Bands \\
\hline \multirow[t]{2}{*}{ NGC2976 } & SPIRE & 1342192106 & 2010 Mar 11 & Large Map & 1076 & \\
\hline & PACS & 1342207170-73 & 2010 Oct 26 & Scan Map & 3624 & $75 \mu \mathrm{m}, 110 \mu \mathrm{m}, 170 \mu \mathrm{m}$ \\
\hline \multirow[t]{2}{*}{ NGC3031 } & SPIRE & 1342185538 & 2009 Oct 6 & Large Map & 5042 & \\
\hline & PACS & $1342186085-86$ & 2009 Oct 17 & Scan Map & 22208 & $75 \mu \mathrm{m}, 170 \mu \mathrm{m}$ \\
\hline \multirow[t]{2}{*}{ NGC3034 } & SPIRE & 1342185537 & 2009 Oct 6 & Large Map & 2418 & \\
\hline & PACS & $1342209350-51$ & 2010 Nov 10 & Scan Map & 6542 & $75 \mu \mathrm{m}, 170 \mu \mathrm{m}$ \\
\hline \multirow[t]{2}{*}{ NGC3077 } & SPIRE & 1342193015 & 2010 Mar 28 & Large Map & 2095 & \\
\hline & PACS & $1342216507-10$ & 2011 Mar 21 & Scan Map & 7356 & $75 \mu \mathrm{m}, 110 \mu \mathrm{m}, 170 \mu \mathrm{m}$ \\
\hline NGC3185/3187/3190 & SPIRE & 1342196668 & 2010 May 18 & Large Map & 1035 & \\
\hline NGC3187/3190 & PACS & $1342207145-48$ & 2010 Oct 25 & Scan Map & 2708 & $75 \mu \mathrm{m}, 110 \mu \mathrm{m}, 170 \mu \mathrm{m}$ \\
\hline \multirow{2}{*}{ NGC3226/3227 } & SPIRE & 1342197318 & 2010 May 30 & Large Map & 2624 & \\
\hline & PACS & $1342221146-47$ & 2010 May 16 & Scan Map & 1178 & $75 \mu \mathrm{m}, 170 \mu \mathrm{m}$ \\
\hline \multirow[t]{2}{*}{ NGC3395/3396 } & SPIRE & 1342209286 & 2010 Nov 9 & Large Map & 999 & \\
\hline & PACS & 1342221104-07 & 2011 May 16 & Scan Map & 2564 & $75 \mu \mathrm{m}, 110 \mu \mathrm{m}, 170 \mu \mathrm{m}$ \\
\hline NGC3424/3430 & SPIRE & 1342195946 & 2010 May 8 & Large Map & 1618 & \\
\hline NGC3448/UGC6016 & SPIRE & 1342185539 & 2009 Oct 6 & Large Map & 1833 & \\
\hline \multirow[t]{3}{*}{ NGC3690/IC694 } & SPIRE & 1342199344 & 2010 Jun 29 & Large Map & 459 & \\
\hline & PACS & 1342210600-05 & 2010 Nov 30 & Scan Map & 2462 & $75 \mu \mathrm{m}, 110 \mu \mathrm{m}, 170 \mu \mathrm{m}$ \\
\hline & PACS & 134211104-05 & 2010 Dec 13 & Scan Map & 524 & $110 \mu \mathrm{m}, 170 \mu \mathrm{m}$ \\
\hline \multirow[t]{2}{*}{ NGC3786/3788 } & SPIRE & 1342223233 & 2011 Jun 28 & Small Map & 169 & \\
\hline & PACS & $1342223319-20$ & 2011 Jun 29 & Scan Map & 104 & $75 \mu \mathrm{m}, 170 \mu \mathrm{m}$ \\
\hline \multirow[t]{2}{*}{ NGC4038/4039 } & SPIRE & 1342188686 & 2009 Dec 29 & Large Map & 710 & \\
\hline & PACS & 1342187836-39 & 2009 Dec 8 & Scan Map & 2662 & $75 \mu \mathrm{m}, 110 \mu \mathrm{m}, 170 \mu \mathrm{m}$ \\
\hline NGC4618/4625 & SPIRE & 1342188755 & 2009 Dec 31 & Large Map & 1052 & \\
\hline NGC4625 & PACS & 1342210468-71 & 2010 Nov 19 & Scan Map & 2708 & $75 \mu \mathrm{m}, 110 \mu \mathrm{m}, 170 \mu \mathrm{m}$ \\
\hline NGC4647/4649 & SPIRE & 1342188778 & 2009 Dec 31 & Large Map & 4295 & \\
\hline \multirow[t]{2}{*}{ M51 } & SPIRE & 1342188589 & 2009 Dec 26 & Large Map & 1577 & \\
\hline & PACS & $1342188328-29$ & 2009 Dec 20 & Scan Map & 4422 & $75 \mu \mathrm{m}, 170 \mu \mathrm{m}$ \\
\hline \multirow[t]{2}{*}{ NGC5394/5395 } & SPIRE & 1342236140 & 2012 Jan 1 & Large Map & 1253 & \\
\hline & PACS & $1342211285-88$ & 2010 Dec 17 & Scan Map & 2200 & $75 \mu \mathrm{m}, 110 \mu \mathrm{m}, 170 \mu \mathrm{m}$ \\
\hline \multirow{2}{*}{ M101 } & SPIRE & 1342188750 & 2009 Dec 30 & Large Map & 9443 & \\
\hline & PACS & 1342198471-74 & 2010 Jun $16-17$ & Scan Map & 38077 & $75 \mu \mathrm{m}, 110 \mu \mathrm{m}, 170 \mu \mathrm{m}$ \\
\hline \multirow[t]{2}{*}{ NGC5474 } & SPIRE & 1342188751 & 2009 Dec 26 & Large Scan & 1052 & \\
\hline & PACS & $13422077178-81$ & 2010 Oct 26 & Scan Map & 2708 & $75 \mu \mathrm{m}, 110 \mu \mathrm{m}, 170 \mu \mathrm{m}$ \\
\hline
\end{tabular}

TABLE 4

Description of GALEX Observations

\begin{tabular}{lllrrr}
\hline \hline \multicolumn{1}{c}{ Galaxy } & \multicolumn{2}{c}{ Tilename } & \multicolumn{2}{c}{ NUV } & \multicolumn{2}{c}{ FUV } \\
& & Date & Exposure (s) & Date & Exposure (s) \\
\hline NGC2976 & GI2_024002_NGC2976_stream & 2006 Jan 04 & 18113.55 & 2006 Jan 04 & 17212.50 \\
NGC3031 & GI1_071001_M81 & 2005 Jan 12 & 29421.55 & 2006 Jan 05 & 14706.70 \\
NGC3034 & NGA_M82 & 2009 Jan 31 & 17311.95 & 2009 Jan 31 & 11527.35 \\
NGC3185/3187/3190 & NGA_NGC3190 & 2004 Jan 30 & 3545.80 & 2005 Feb 19 & 1299.15 \\
NGC3395/3396/3424/3430 & GI1_078004_NGC3395 & 2006 Mar 23 & 2666.15 & 2006 Mar 23 & 1500.10 \\
NGC3448/UGC6016 & AIS_92 & 2004 Feb 4 & 423.00 & 2004 Apr 21 & 143.00 \\
NGC3690/IC694 & AIS_99 & 2007 Feb 13 & 211.00 & 2007 Feb 13 & 211.00 \\
NGC3786/3788 & AIS_111 & 2007 Feb 20 & 103.05 & 2007 Feb 20 & 103.05 \\
NGC4038/4039 & NGA_Antennae & 2004 Feb 22 & 1541.30 & 3004 Feb 22 & 1541.30 \\
NGC4618/4625 & NGA_NGC4625 & 2004 Apr 5 & 3259.00 & 2004 Apr 5 & 3259.00 \\
NGC4647/4649 & GI1_109003_NGC4660 & 2005 Apr 30 & 3113.25 & 2008 Apr 23 & 1624.10 \\
M51 & GI3_050006_NGC5194 & 2007 May 29 & 10216.20 & 2007 May 29 & 10216.20 \\
NGC5394/5395 & GI1_026018_Arp84 & 2006 Apr 12 & 4268.65 & 2007 May 30 & 2811.40 \\
M101 & GI3_050008_NGC5457 & 2008 Apr 4 & 13294.40 & 2008 Apr 4 & 13293.4 \\
NGC5474 & NGA_NGC5474 & 2003 Jun 19 & 1610.00 & 2003 Jun 19 & 1610.10 \\
\hline
\end{tabular}


TABLE 5

DESCRIPTION OF Swift UVOT OBSERVATIONS

\begin{tabular}{cccccc}
\hline \hline & \multirow{2}{*}{ ObsID } & Date & Exposure Times $(\mathrm{s})$ \\
uvw2 & uvm2 & uvw1 \\
\hline NGC3226/3227 & 00031280001 & 2008 Nov 4 & 342 & 249 & 352 \\
NGC3226/3227 & 00031280002 & 2008 Nov 5 & 704 & 511 & 346 \\
NGC3226/3227 & 00031280003 & 2008 Nov 12 & 692 & 424 & 372 \\
NGC3226/3227 & 00031280004 & 2008 Nov 13 & 744 & 538 & 372 \\
NGC3226/3227 & 00031280005 & 2008 Nov 21 & 744 & 522 & 381 \\
NGC3226/3227 & 00031280006 & 2008 Nov 22 & 763 & 137 & 381 \\
NGC3226/3227 & 00031280007 & 2008 Nov 25 & 763 & 531 & 381 \\
NGC3226/3227 & 00031280008 & 2008 Nov 27 & 763 & 196 & 246 \\
NGC3226/3227 & 00031280009 & 2008 Dec 2 & 0 & 0 & 293 \\
NGC3226/3227 & 00031280010 & 2008 Dec 3 & 274 & 349 & 126 \\
\hline NGC3424/3430 & 00091132001 & 2011 Apr 16 & 0 & 0 & 1976 \\
NGC3424/3430 & 00091132003 & 2011 Jun 28 & 0 & 0 & 0 \\
NGC3424/3430 & 00091132004 & 2011 Jul 4 & 0 & 80 & 1315 \\
NGC3424/3430 & 00091132005 & 2011 Jul 7 & 302 & 0 & 0 \\
NGC3424/3430 & 00091132006 & 2011 Jul 8 & 0 & 1877 & 0 \\
NGC3424/3430 & 00091132007 & 2011 Oct 7 & 750 & 988 & 0 \\
NGC3424/3430 & 00091132008 & 2011 Oct 10 & 0 & 0 & 0 \\
\hline
\end{tabular}

Note. - The Swift observation ID number (Col. 2) and the start date of each observation (Col. 3) are given for each observation of each object for which observations with minimal coincidence losses exist. Exposure times in the each filter are given in Col. 4-6.

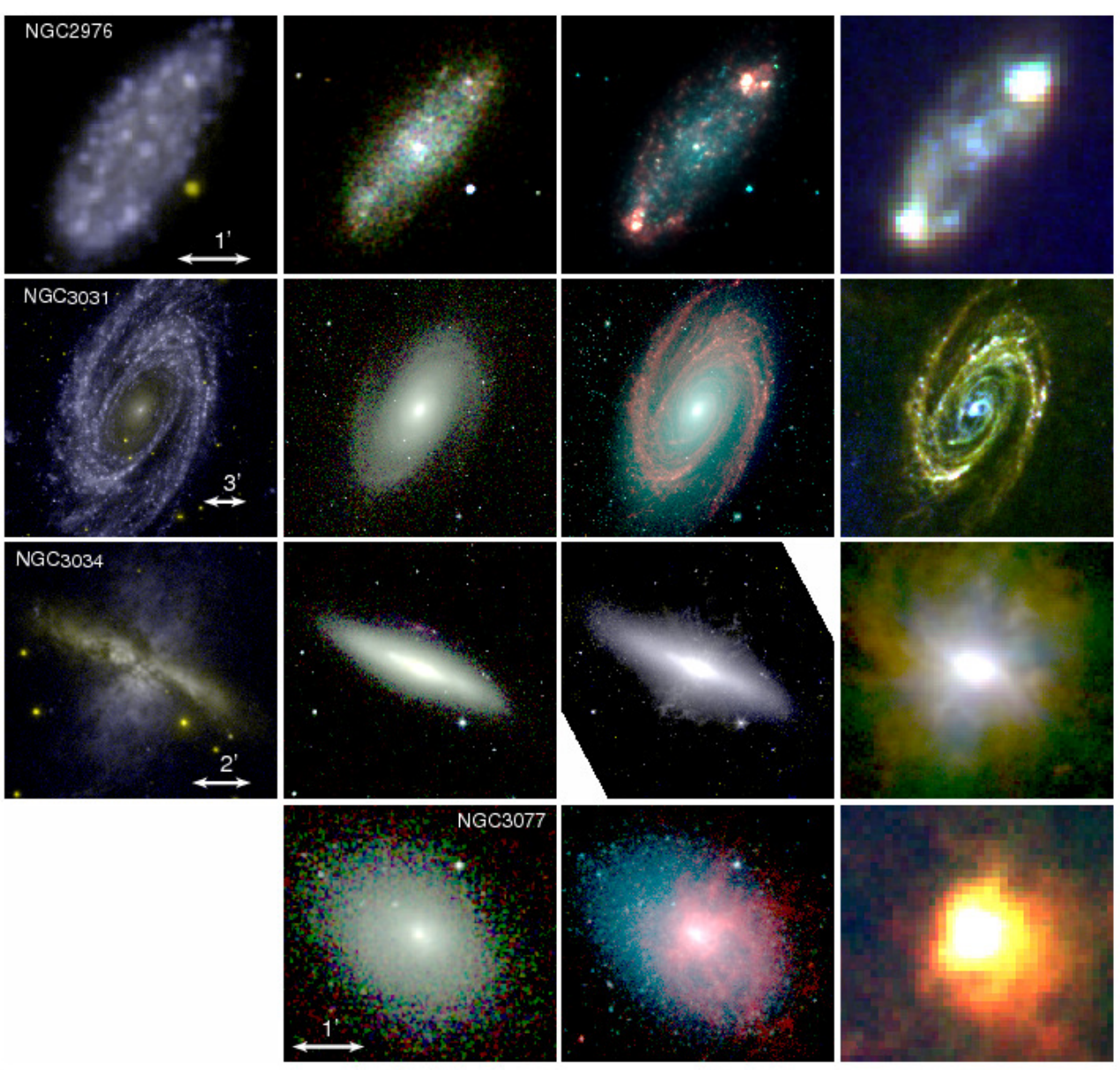

FIG. 1.- NGC 2976, NGC 3031, NGC 3034, and NGC 3077 (from top to bottom) as observed, from left to right, by GALEX (NUV in yellow; FUV in blue), 2MASS ( $J$ in blue, $H$ in green, and $K s$ in red), IRAC (3.6 $\mu \mathrm{m}$ in blue, $4.5 \mu \mathrm{m}$ in green, and $8.0 \mu \mathrm{m}$ in red), and Herschel (PACS $75 \mu \mathrm{m}$ in blue, PACS $170 \mu \mathrm{m}$ in green, and SPIRE $250 \mu \mathrm{m}$ in red). The longer wavelength IRAC observations of NGC 3034 were saturated, so $4.5 \mu \mathrm{m}$ is shown in yellow instead. NGC 3077 was not observed by either GALEX or Swift. At the distance of these galaxies, $1^{\prime} \approx 1.1 \mathrm{kpc}$. 


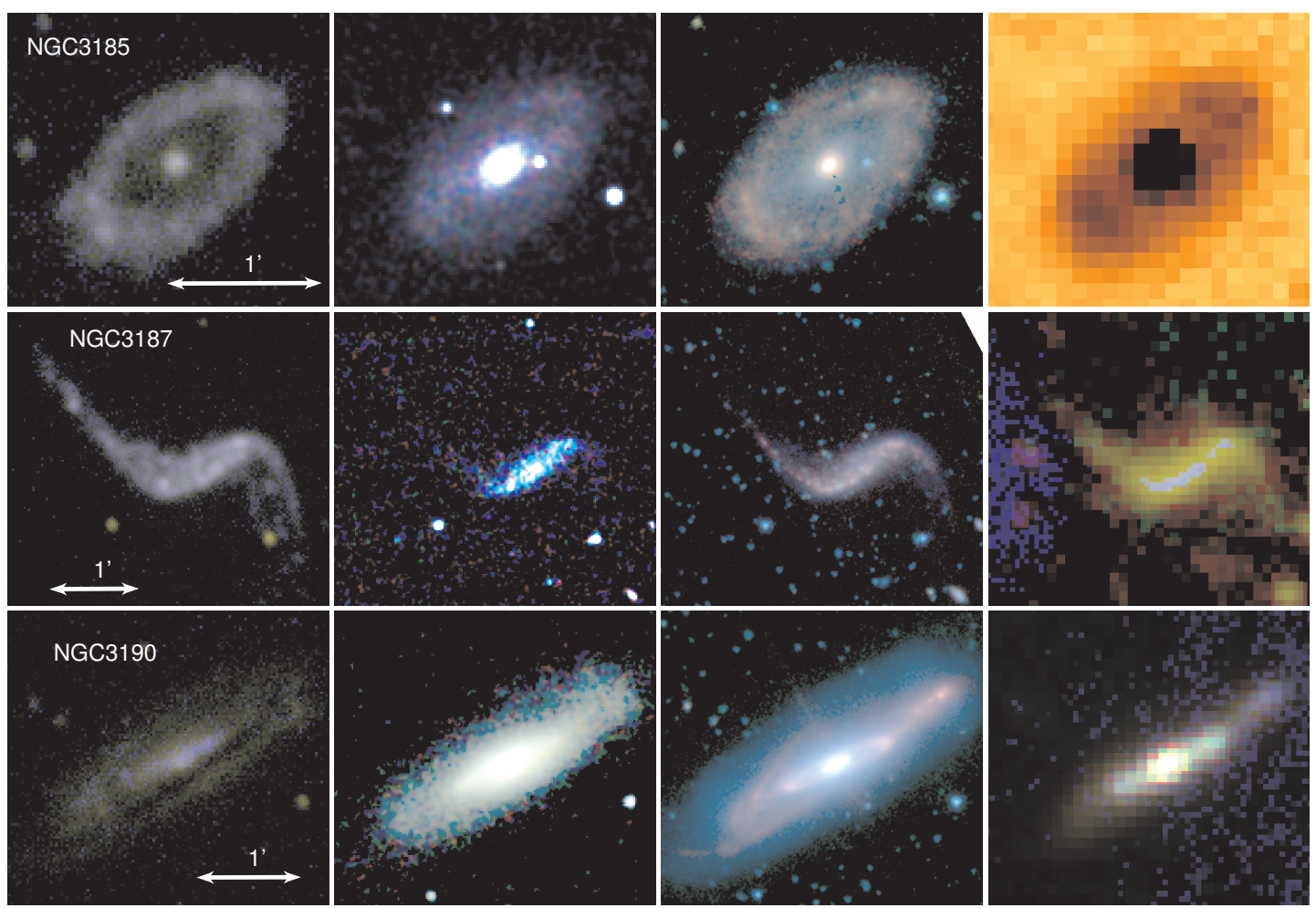

Fig. 2.- As Figure 1, but for NGC 3185, NGC 3187, and NGC 3190. NGC 3185 was not observed by PACS, the right image only shows the SPIRE $250 \mu \mathrm{m}$ image in which darker pixels have higher flux.. At the distance of these galaxies, 1 ' is approximately $6-7$ kpc.

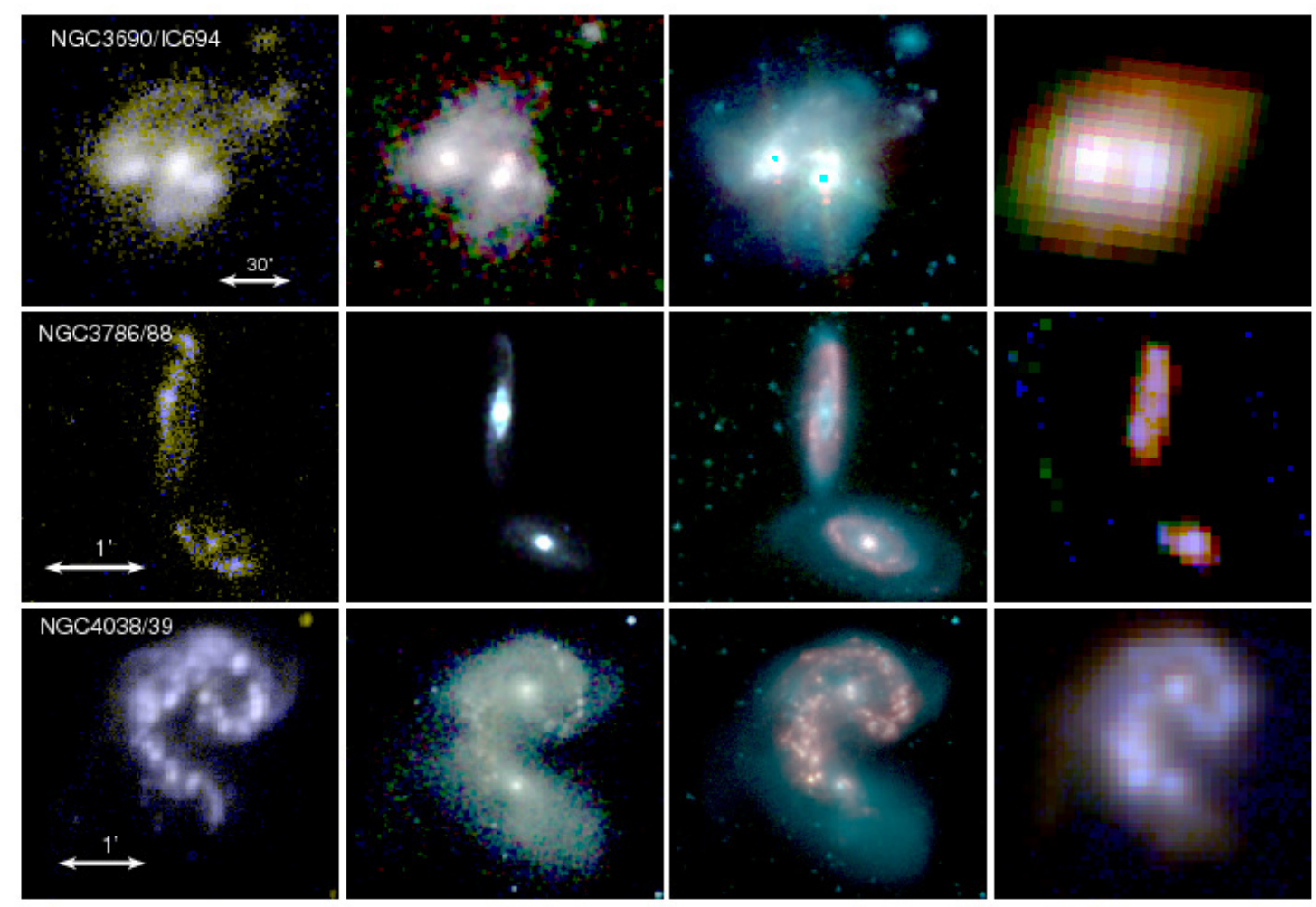

FIG. 3.- As Figure 1, but for NGC 3690/IC694, NGC 3786 (bottom)/NGC 3788 (top), and NGC 4038/4039. The $8 \mu \mathrm{m}$ IRAC image of NGC 3690/IC 694 is saturated in the nuclei of the two galaxies, resulting in the blue-green artifacts. At the distance of these galaxies, 1 ' is approximately $14 \mathrm{kpc}$ (NGC 3690), 11-12 kpc (NGC 3786/3788), and $7.4 \mathrm{kpc}$ (NGC 4038/4039). 

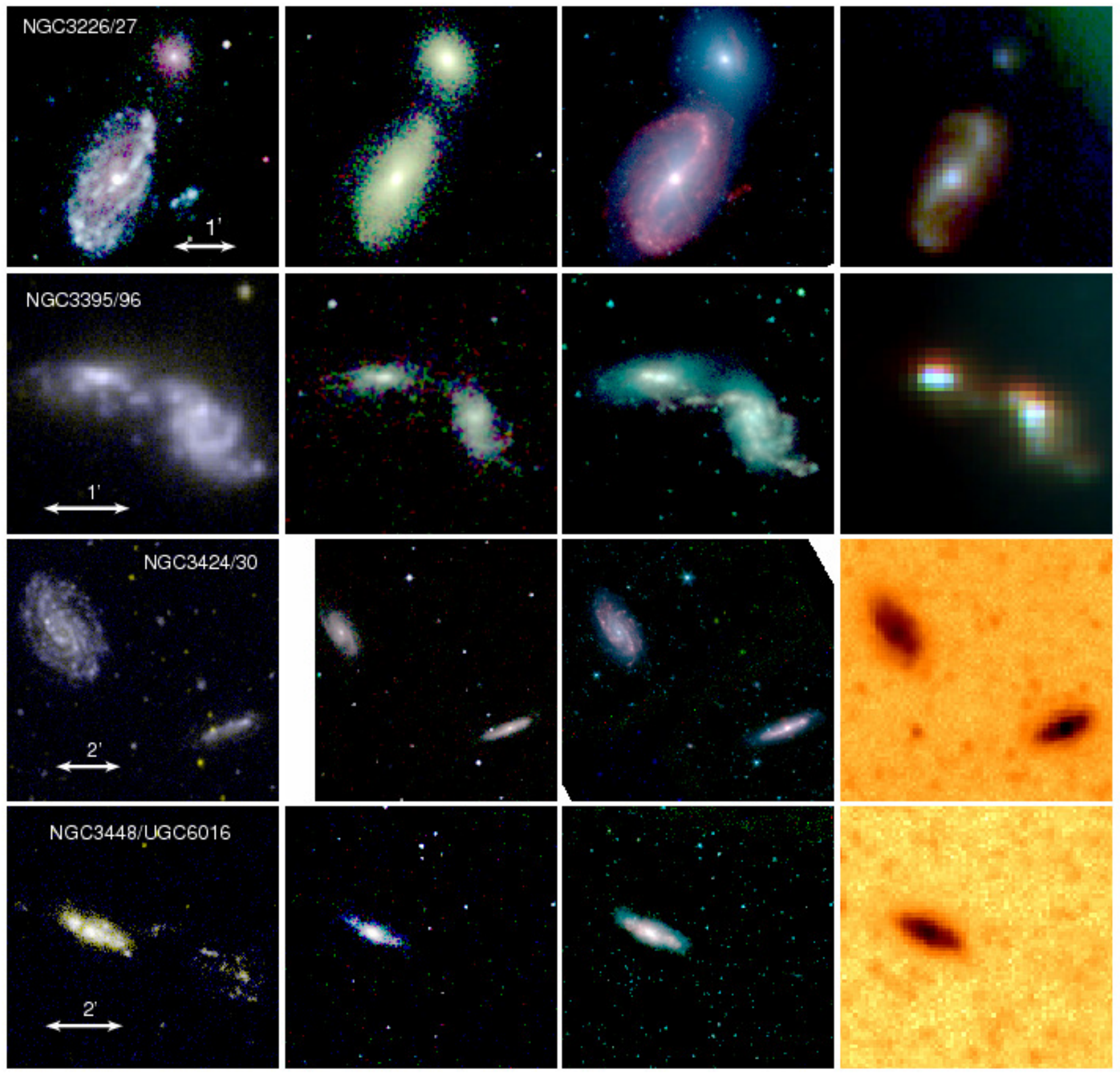

Fig. 4.- As Figure 1, but for NGC 3226 (upper)/NGC 3227 (lower), NGC 3395 (right)/NGC 3396 (left), NGC 3424 (right)/NGC 3430 (left), and NGC 3448 (left)/UGC 6016 (right). NGC $3226 / 3227$ was not observed by GALEX but by Swift. Their left image show the Swift observations through the UVW1 filter in blue, the UVM2 filter in green, and the UVW2 filter in red. NGC 3424/30 and NGC 3448/UGC 6016 were not observed with PACS, so the right image only shows the SPIRE $250 \mu \mathrm{m}$ image as in Figure 2. UGC 6016, while having significant extended diffuse emission in the UV, is not well detected in the IR bands. At the distance of these galaxies 1 ' is approximately 6-8 kpc.

et al. (2008) recently compiled redshift-independent distances for nearby galaxies with velocities less than 3000 $\mathrm{km} \mathrm{s}^{-1}$ using alternate methods including Cepheids (Freedman et al. 2001), the luminosity of stars at the tip of the red giant branch (Karachentsev et al. 2006), surface brightness fluctuations (Tonry et al. 2001), and the Tully-Fisher relation (Tully \& Fisher 1977). Distances to additional galaxies based on their group or cluster association are given in the Extra-galactic Distance Database $^{11}$ (EDD; Tully, 2010, private communication).
Twenty-six of our galaxies have distance moduli given by either Tully et al. (2008), Tully (1994), or EDD. For the five galaxies lacking distance moduli, we obtained heliocentric velocities from the PSCz catalog (Saunders et al. 2000; NGC 3690/IC 694, NGC 5394, and NGC 5395) and RC3 (UGC 6016), which we corrected to account for the velocity field of Virgo, the Great Attractor, and the Shapley supercluster, following Mould et al. (2000). Distances were then calculated assuming $H_{0}=72 \mathrm{~km} \mathrm{~s}^{-1}$ $\mathrm{Mpc}^{-1}$. The distances are given in Table 1 .

11 http://edd.ifa.hawaii.edu 

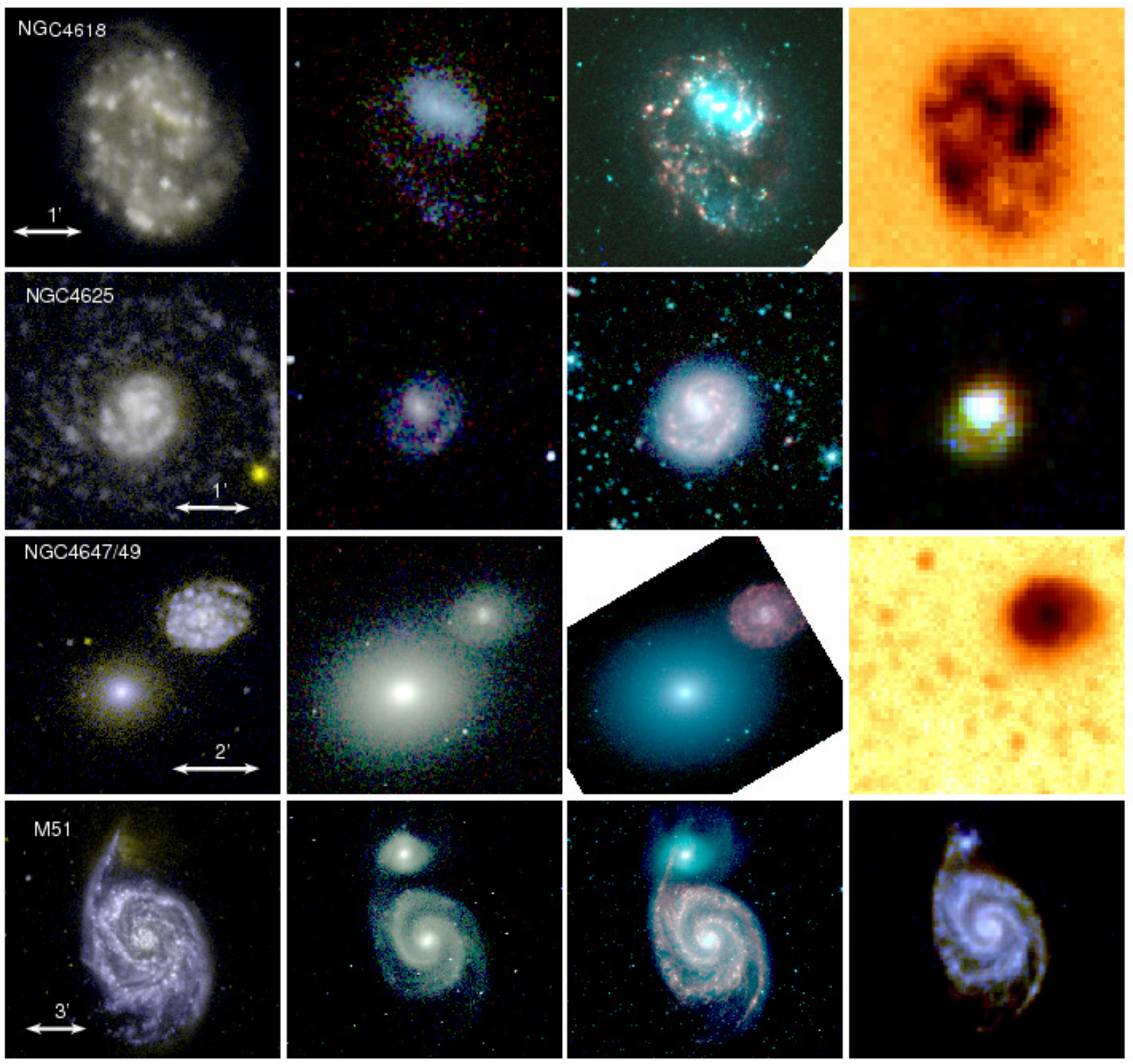

FIG. 5.- As Figure 1, but for NGC 4618, NGC 4625, NGC 4647 (right)/NGC 4649 (left), and M51. The right images of NGC 4618 and NGC $4647 / 49$ only show the SPIRE $250 \mu \mathrm{m}$ image. At the distance of these galaxies, 1 ' is approximately $2.1-2.4 \mathrm{kpc}$ (NGC $4618 / 4625)$, 5 kpc (NGC 4647/4649), and $2.2 \mathrm{kpc}$ (M51).

\subsection{Infrared Photometry \\ 3.2.1. Spitzer Observations}

The IRAC (Fazio et al. 2004) and MIPS (Rieke et al. 2004) $24 \mu \mathrm{m}$ observations were taken as part of a variety of programs, including the main SIGS program (PID 20140; P.I. A. Zezas), which also observed galaxy groups that had not previously been observed. The observation parameters are given in Table 2. The IRAC Basic Calibrated Data (BCD) were retrieved from the Spitzer archive and cleaned before being coadded into mosaics with 0.6" pixels using IRACproc (Schuster et al. 2006). The MIPS $24 \mu \mathrm{m}$ BCDs were merged to form mosaics with $2.45^{\prime \prime}$ pixels using the Mosaicker and Point Source Extractor package (MOPEX; Makovoz \& Khan 2005).
The reduction of these data will be described in detail in Brassington et al. (2013, in preparation). While the pipeline versions range from $\mathrm{S} 13-\mathrm{S} 18$, the difference between the pipelines are minor and do not impact significantly the photometry ${ }^{12}$. The pipeline version for each galaxy is given in Table 2 .

\subsubsection{Herschel Observations}

The parameters for the Herschel SPIRE (Griffin et al. 2010) and PACS (Poglitsch et al. 2010) observations are given in Table 3. The Herschel data were taken as part of two Science Demonstration Phase programs (P.I.s C.

12 http://irsa.ipac.caltech.edu/data/SPITZER/docs/irac/ iracinstrumenthandbook/79/ 

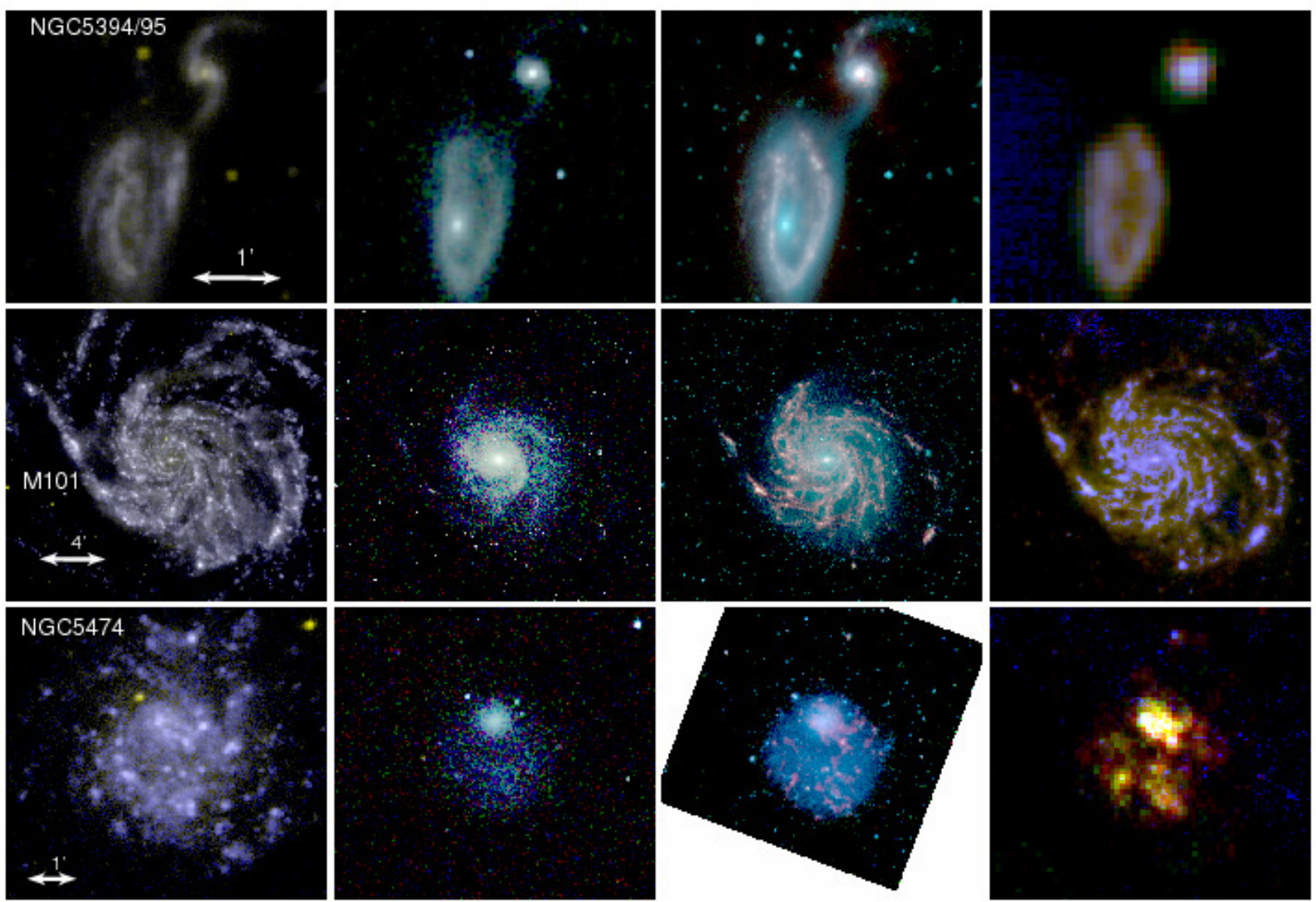

Fig. 6. - As Figure 1, but for NGC 5394 (top)/NGC 5395 (bottom), M101, and NGC 5474. At the distances of these galaxies, 1' is approximately $16 \mathrm{kpc}$ (NGC 5394/5395) and 1.7-2.0 kpc (M101/NGC 5474).

Wilson and S. Eales), four Key Project programs (P.I.s R. Kennicutt, S. Eales, C. Wilson, and E. Sturm), and one Guaranteed Time program (P.I. L. Spinoglio). All of the galaxies were observed by SPIRE at $250 \mu \mathrm{m}, 350 \mu \mathrm{m}$, and $500 \mu \mathrm{m}$; this was part of the selection criteria of this sample. Approximately $50 \%$ of the sample were observed in all three PACS bands and an additional $\sim 25 \%$ were observed at $75 \mu \mathrm{m}$ and $170 \mu \mathrm{m}$.

The data were retrieved from the Herschel Science Archive and processed using the calibration trees of version 8.0.1 of the Herschel Interactive Processing Environment (HIPE; Ott 2010). This processing was accomplished using the default pipeline scripts available through HIPE to make Large Map mode mosaics for the SPIRE data and extended source mosaics with MadMap for PACS data. We discuss additional details regarding the processing of PACS data in Appendix B1.

\subsubsection{MASS Observations}

NIR mosaics of the sample galaxies observed as part of the 2MASS (Skrutskie et al. 2006) were retrieved from the NASA/IPAC Infrared Science Archive ${ }^{13}$, and from the Large Galaxy Atlas (Jarrett et al. 2003) when possible. The counts measured in the images were converted

13 NASA/IPAC Infrared Science Archive is operated by the Jet Propulsion Laboratory, California Institute of Technology, under contract with NASA. to Janskys using the zero points of Cohen et al. (2003). We compared our fluxes measured in the apertures described in $\S 3.4$ to the total fluxes given in NED from Jarrett et al. (2003) and the 2MASS Extended Object Catalog and found good agreement.

\subsubsection{Ancillary IRAS Photometry}

IRAS photometry was obtained from the HIRES Atlas (Surace et al. 2004), the IRAS Revised Bright Galaxy Sample (Sanders et al. 2003), the IRAS Bright Galaxy Sample (Soifer et al. 1989), and the Faint Source Catalogue (Moshir et al. 1990). The latter three catalogs present photometry derived from the native IRAS beam size of $2^{\prime}-5^{\prime}$; this can be problematic for systems in close interaction phases. We therefore preferentially used the HIRES Atlas, which was reprocessed with $30^{\prime \prime}-1$ '.5. In the one system where only low-resolution photometry is available and the galaxies are close enough for contamination to occur, we do not include the IRAS photometry in our analysis.

\subsection{Ultraviolet Photometry \\ 3.3.1. GALEX Observations}

Twenty-eight of our sample galaxies were observed by GALEX; three sources within the sample (NGC 3226, NGC 3227, and NGC 3077), however, were not observed due to the presence of nearby bright stars. For the galaxies with GALEX photometry, mosaics of the longest ob- 
servations were retrieved from the Mikulski Archive for Space Telescopes ${ }^{14}$ using GalexView version 1.4.6. The details of those observations are given in Table 4 . The NUV observation of NGC 3690/IC 694 was reprocessed by $D$. Neill at our request to correct a masking problem. We use the conversions from count rate to fluxes provided by Goddard Space Flight Center $(2004)^{15}$.

\subsubsection{Swift UVOT Observations}

Most of the gaps in the GALEX coverage can be filled in with data from the Swift UVOT telescope, which has three UV filters that bracket the GALEX NUV filter in mean wavelength. Two of the three galaxies lacking GALEX data, NGC 3226 and NGC 3227, were observed by UVOT. Unfortunately, NGC 3077's nearby bright star exceeded the tolerances of this telescope as well. We originally planned to use existing UVOT photometry for all our sample. We obtained the raw data and exposure maps from the Swift archive for the seventeen galaxies with UVOT data and coadded the observations into one mosaic and exposure map per UV filter per interacting system. However, as described by Hoversten et al. (2011), the photon-counting nature of the Swift detectors makes them vulnerable to coincidence losses, which become significant when the count rate is greater than 0.007 counts per second per pixel. We calculated count rate maps to determine where coincidence losses need to be taken into account. Due the difficulties associated with coincidence losses in extended sources, described in greater length in Appendix B2, we opted only to use the UVOT data for the missing GALEX objects NGC 3226 and NGC 3227. We added one test case, NGC 3424, to confirm that the UVOT data yielded fluxes consistent with GALEX and found good agreement. The details of the observations of these three galaxies are given in Table 5. To convert the count rate to fluxes, we used the conversion assuming a stellar spectrum described in Breeveld et al. (2010).

\subsection{Aperture and Uncertainty Determination}

For consistency, we used matched apertures across all wavebands in our photometric analysis. Generally, the IR emission of galaxies is more extended that their UV emission. However, some of the galaxies are more extended in the UV than in the IR (e.g. NGC 3430). We used the SExtractor algorithm (Bertin \& Arnouts 1996) to determine Kron apertures in both the NUV and the $3.6 \mu \mathrm{m}$ IRAC images. In all cases, the larger of the two apertures was then used to measure the integrated galaxy flux at all wavelengths in order to obtain flux from a consistent area of each galaxy across our wavelength range. The size and position angle of each aperture as well as on which image it was determined is given in Table 1. Background regions were selected to mimic the content of background and foreground objects in the aperture on the outskirts of the galaxies. Once the aperture was selected, flux densities in the aperture and background

\footnotetext{
14 STScI is operated by the Association of Universities for Research in Astronomy, Inc., under NASA contract NAS5-26555. Support for MAST for non-HST data is provided by the NASA Office of Space Science via grant NNX09AF08G and by other grants and contracts.
}

15 http://galexgi.gsfc.nasa.gov/docs/galex/FAQ/counts_background.htmipsinstrumenthandbook/50/ regions were measured using the analysis tools of the SAOImage DS9 (Joye \& Mandel 2003).

Due to the proximity of some members of the same interacting system, their apertures can overlap. We dealt with these situations in one of three ways. For significantly overlapping systems (NGC 4038/4039, NGC 3690/IC 694, and NGC 3395/3396), separate apertures could not be robustly determined. In these cases, we treated the combined system as a single object. Second, there were two systems (M51 A/B and NGC 5394/5495) where the aperture for the smaller galaxy was mostly contained within the aperture of the larger galaxy, but it was clear that the emission in the overlap area came from the smaller galaxy. In these cases, we subtracted the emission and area of the overlap region from that of the larger aperture. Third, there were three systems (NGC $3226 / 3227$, NGC 3786/3788, and NGC 4647/4649) where the aperture overlapped but without significant contamination. In these cases, we extrapolated the expected flux in the overlap area from the surface brightness in the rest of the elliptical aperture at the same radii.

The Spitzer fluxes required aperture corrections. We determined the effective radius of the elliptical aperture $^{16}$ and used the extended source flux corrections given in the IRAC Instrument Handbook ${ }^{17}$. For the MIPS $24 \mu \mathrm{m}$ aperture corrections, we interpolated between the aperture corrections given in the MIPS Instrument Handbook ${ }^{18}$. The GALEX data were corrected for obscuration due to Milky Way dust using the extinction laws given by Wyder et al. (2005).

Uncertainties in the absolute fluxes are the sum in quadrature of a statistical uncertainty and a calibration uncertainty. The Spitzer bandpass uncertainties are typically dominated by the calibration uncertainty of $3 \%$ for IRAC (Cohen et al. 2003) and 4\% for MIPS $24 \mu \mathrm{m}$ (Engelbracht et al. 2007). We used a calibration uncertainty of $10 \%$ for the GALEX data (Goddard Space Flight Center 2004) and a 5-15\% uncertainty for the Swift bands (Poole et al. 2008), and the statistical uncertainty is calculated using Poisson statistics. We used a 7\% calibration uncertainty for the SPIRE bandpasses (Swinyard et al. 2010) and $10 \%$ for the PACS bandpasses (Paladini et al. 2012) and followed Dale et al. (2012) in calculating the statistical uncertainty. The photometry results for GALEX, Swift, and 2MASS; Spitzer; and Herschel are provided in Table 6-8, respectively. When flux is not determined significantly, we provide $3 \sigma$ upper limits, but we do not provide lower limits in cases of saturated images. The additional photometry from the literature is given in Table 9.

\section{SED FITTING WITH MAGPHYS}

\subsection{Fitting Process}

To estimate SFR, specific star formation rates (sSFRs), stellar and dust masses, and dust temperatures, we used the SED fitting code MAGPHYS (da Cunha et al. 2008). MAGPHYS fits SEDs with a combination of UV-NIR stellar spectral libraries from Bruzual \& Charlot (2003)

\footnotetext{
${ }^{16} \mathrm{r}_{\text {eff }}=\sqrt{a b}$ for semi-major axis $a$ and semi-minor axis $b$

$17 \mathrm{http}$ ///irsa.ipac.caltech.edu/data/SPITZER/docs/irac/ iracinstrumenthandbook/30/

18 http://irsa.ipac.caltech.edu/data/SPITZER/docs/mips/
} 


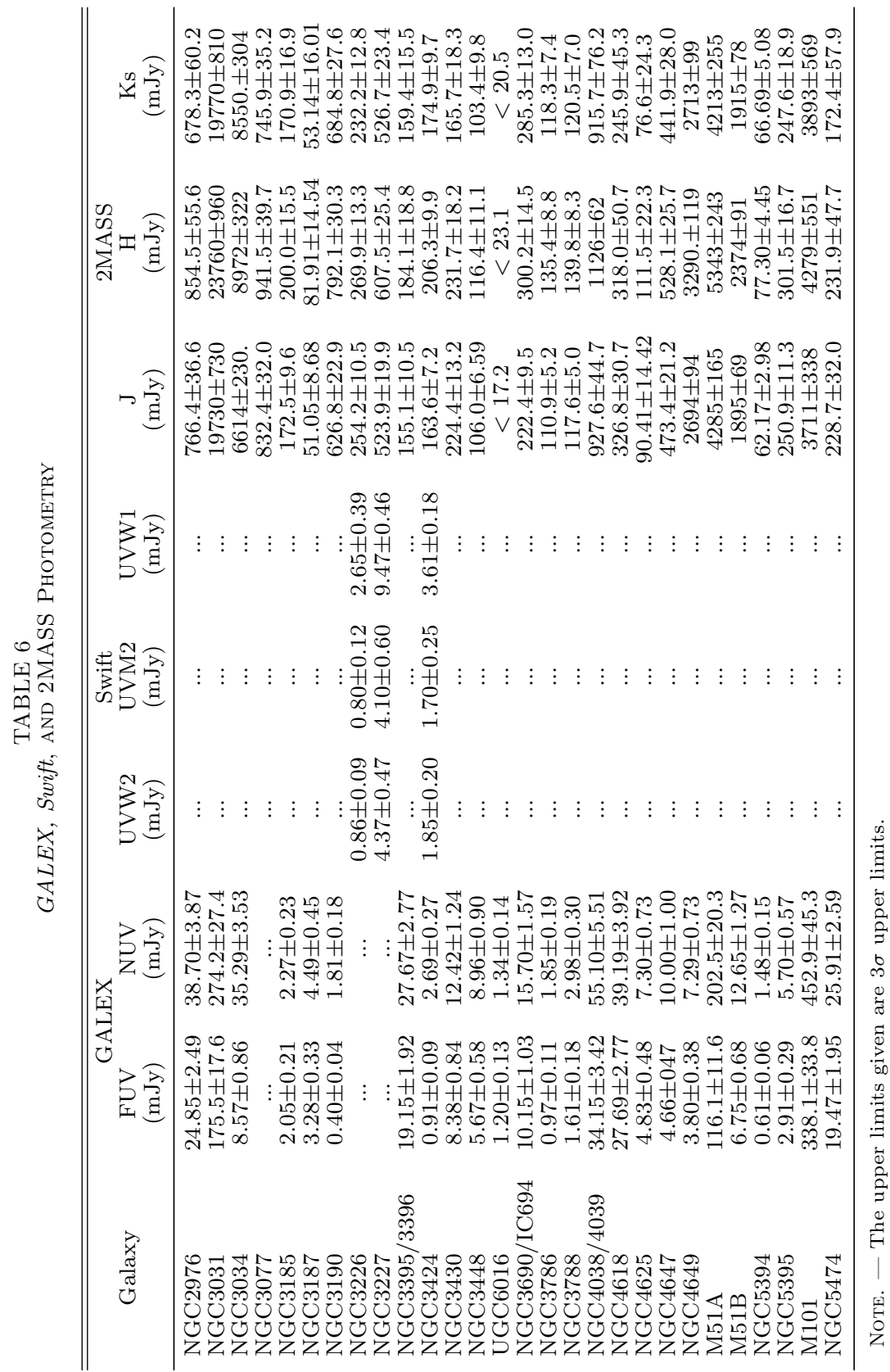




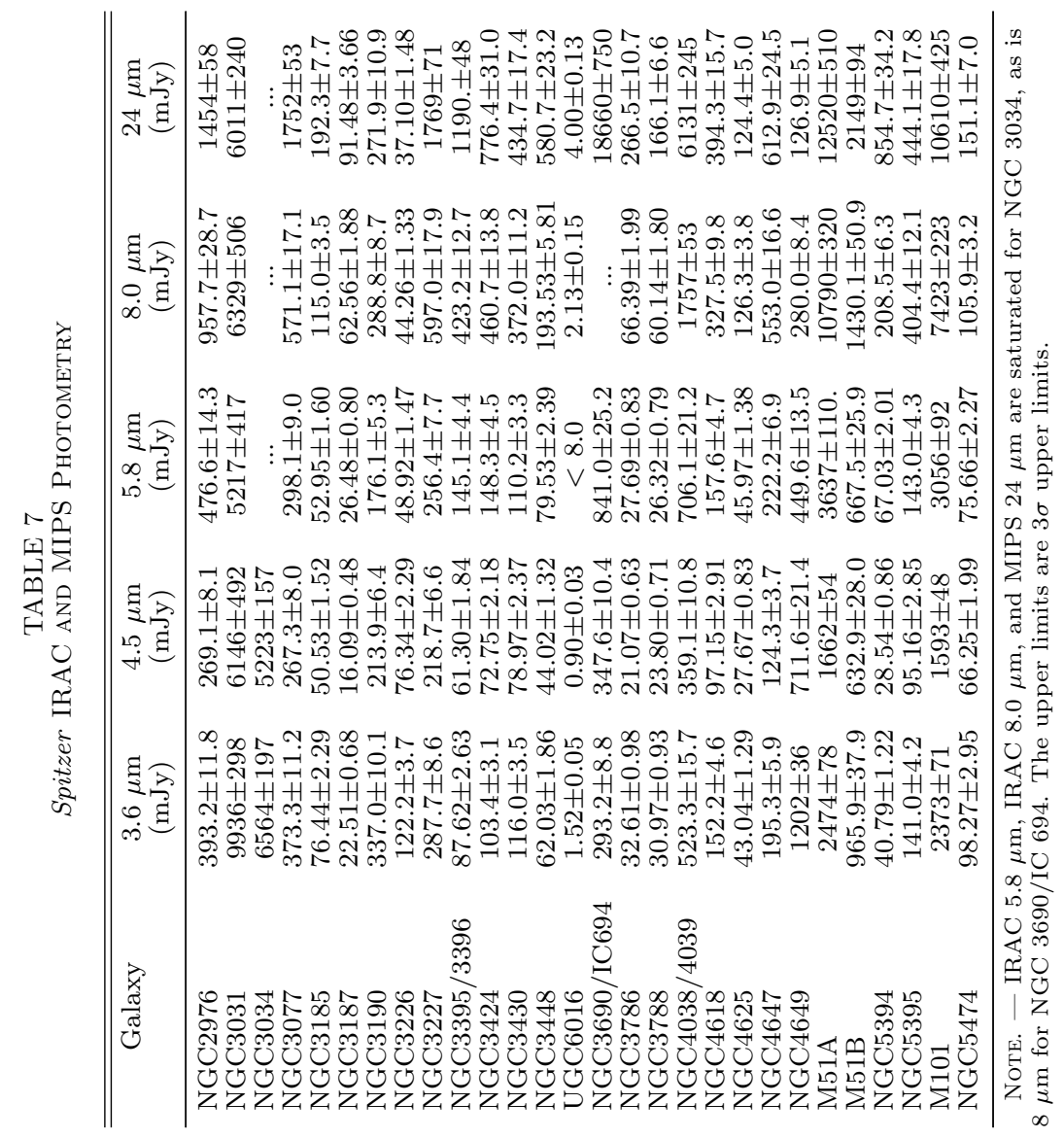




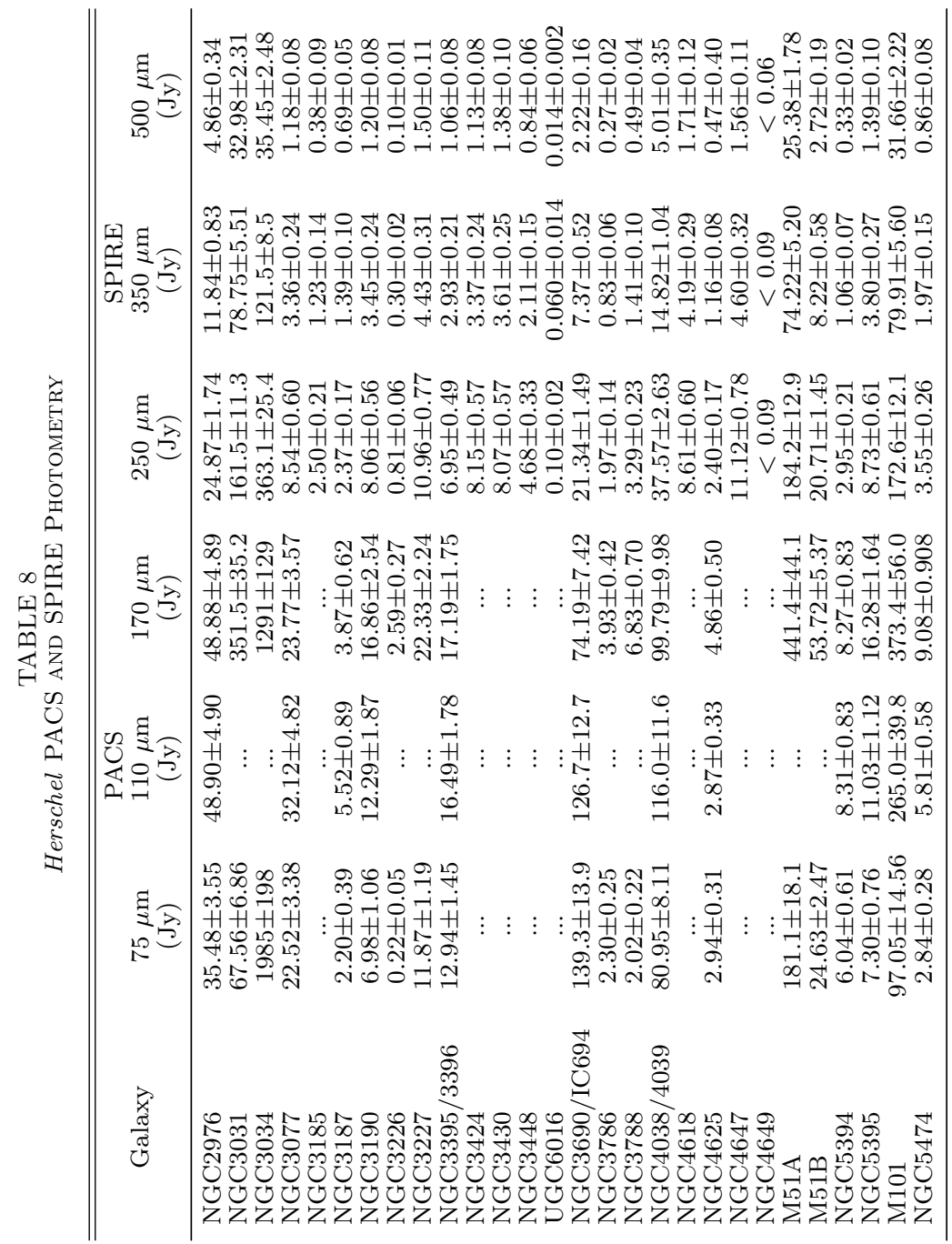




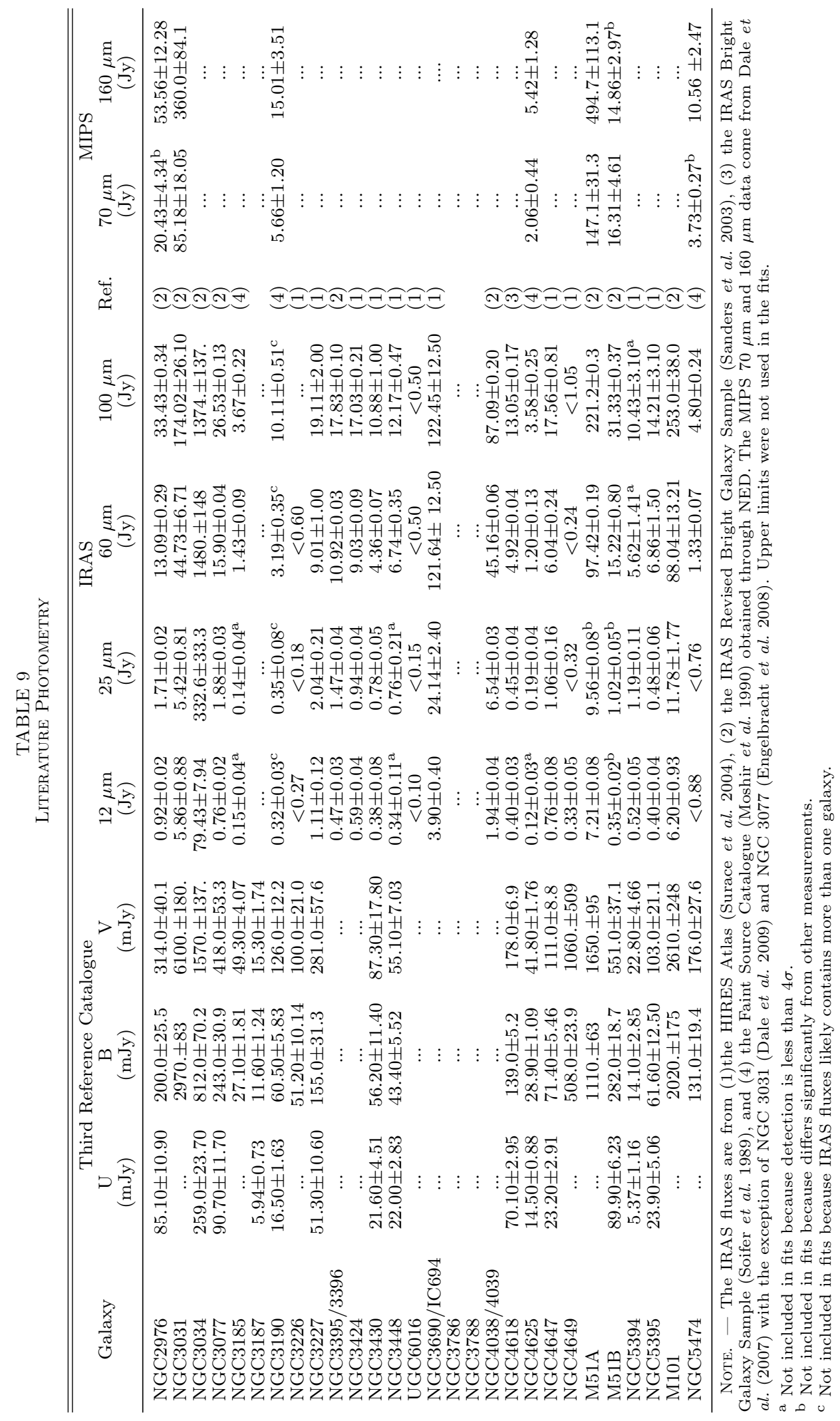



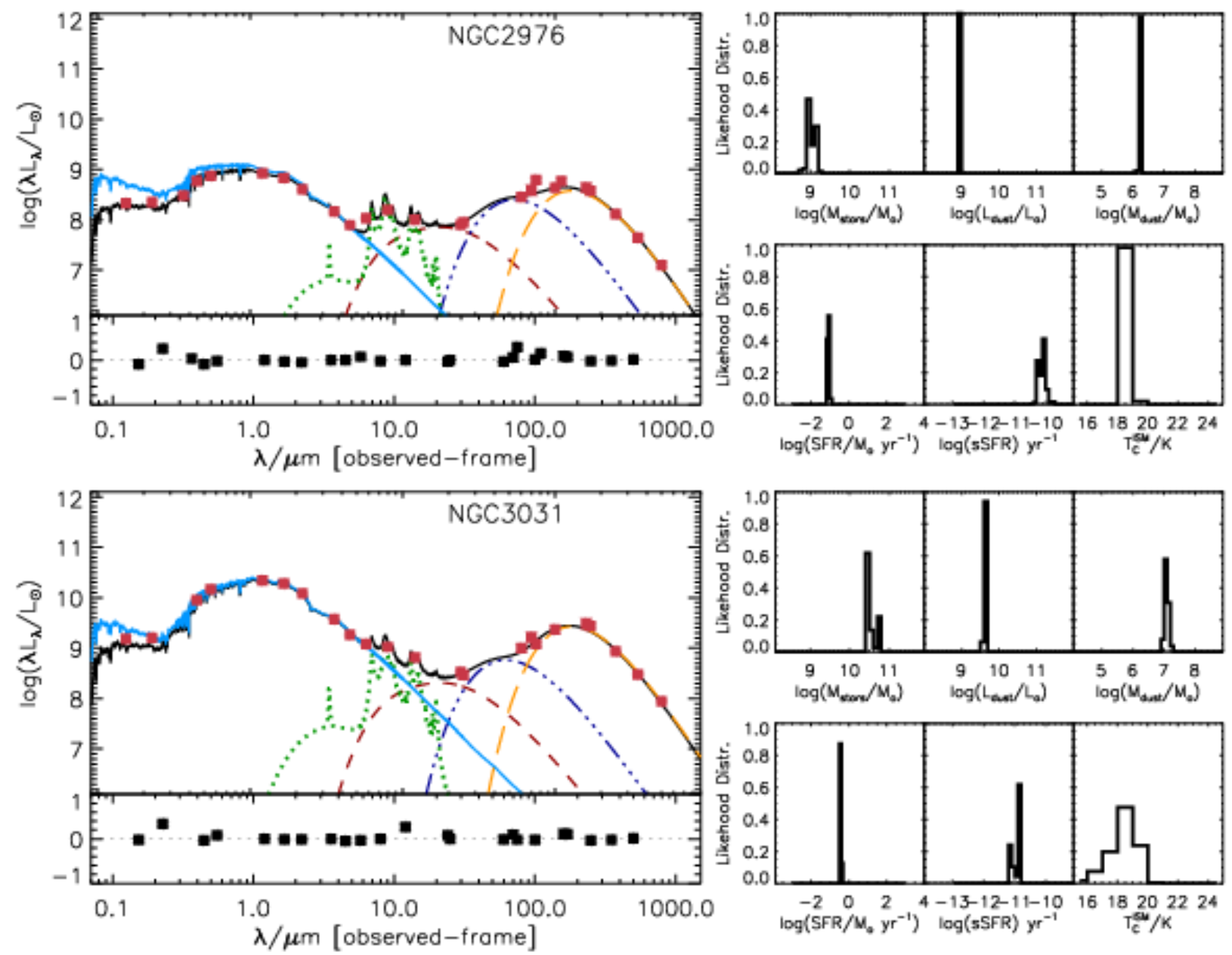

FIG. 7.- SEDs for NGC 2976 (top) and NGC 3031 (bottom) with data shown as red points, the best fit model plotted in black, and the stellar emission in the absence of dust shown in blue. The components of the infrared emission are over plotted: PAH emission (dotted, green line), MIR emission at $130 \mathrm{~K}$ and $250 \mathrm{~K}$ (red, dashed line), warm 30-60 K dust emission (dot-dashed, purple line), and cold 15-25 $\mathrm{K}$ dust emission (long dashed, orange line). Below the fitted SED is plotted the fractional difference between the model and data. To the right of the SED, we plot a subset of the probability distribution functions (PDFs) of the fitted parameters for (from left to right): stellar mass, dust luminosity, and dust mass (top) and SFR, sSFR, and cold dust temperature (bottom).

and a simple, physically-motivated model for IR emission from dust developed in da Cunha et al. (2008). It models the ISM as a mix of diffuse dust interspersed with denser, warmer stellar birth clouds. MAGPHYS also includes a set of UV-NIR libraries that modify the Bruzual \& Charlot (2003) population synthesis with the Bruzual (2007) population synthesis, which provides different treatment of post-Asymptotic Giant Branch (AGB). We fit our UV to FIR SEDs with MAGPHYS with and without the post-AGB modifications and found consistent results; from here on, we only use the results with the earlier Bruzual \& Charlot (2003) libraries as their treatment of the post-AGB stars is more consistent with current understanding (e.g., Zibetti et al. 2012). The IR dust libraries have five components: a fixed polycyclic aromatic hydrocarbon $(\mathrm{PAH})$ spectrum shape derived from the M17 SW star-forming region (Madden et al. 2006), a NIR continuum associated with the PAH emission modeled by a modified blackbody $(\beta=1)$ at $850 \mathrm{~K}$, a hot MIR continuum modeled by the sum of two modified blackbodies $(\beta=1)$ at $130 \mathrm{~K}$ and $250 \mathrm{~K}$, a warm $(30-60 \mathrm{~K})$ dust component modeled as a modified blackbody $(\beta=1.5)$, and a cold $(15-25 \mathrm{~K})$ dust com- ponent modeled as a modified blackbody $(\beta=2)$. The warm dust component is assumed to exist both in the diffuse ISM and in denser birth clouds, while the cold dust exists only in the diffuse ISM. MAGPHYS determines probability distribution functions (PDFs) for the fitted parameters by combining UV-NIR and IR spectral libraries such that the energy absorbed in the UV/visible regime is re-emitted in the IR. It gives both the bestfit obscured SED and the associated unobscured stellar SED.

We input the measured and literature fluxes in our set of 28 filters to MAGPHYS and examined the variation in derived galaxy properties including dust luminosity, SFR, sSFR, stellar and dust mass, and dust temperatures and discuss the results below. Then we performed five additional fits: one without UV photometry, one without SPIRE photometry, one without any photometry at wavelengths $\lambda \geq 30 \mu \mathrm{m}$, one without either UV or SPIRE photometry, and one with only UBV, 2MASS, and IRAC photometry. For these fits for each galaxy, we examined the median and $68 \%$ confidence interval for the fitted parameters to determine the influence of the particular dataset on the value of (and constraints on) 

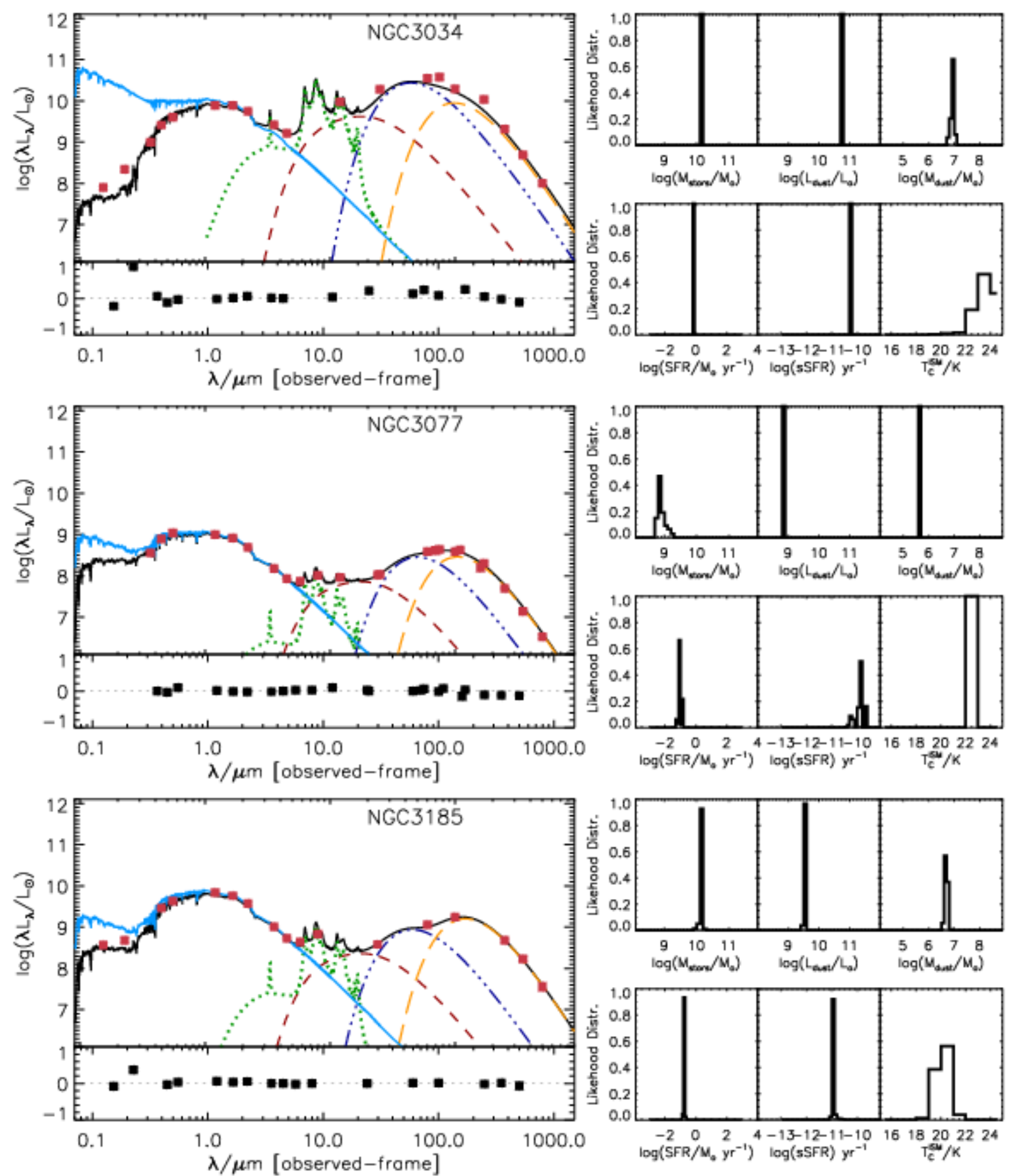

FIG. 8.- As Figure 7, but for NGC 3034 (top), NGC 3077 (middle), and NGC 3185 (bottom).

these properties, as we elaborate below. For the noninteracting galaxies, we only performed the fit with all of the available photometry.

\subsection{SED Fits}

Figures $7-16$ show the SEDs along with the best-fit models for our interacting galaxies. The contributions of the different components of the IR model described above are also shown. The median and $68 \%$ confidence interval of these parameters are given in Table 10 . Just as the galaxies exhibit a variety of UV versus IR morphologies (Figures 1-6), the SEDs have a corresponding range of relative UV, NIR, and FIR emission. For example, some galaxies (e.g., NGC 3190 or M51B) have very little UV flux in comparison with their visible and IR flux, while others (e.g., NGC 3187) have almost as much UV flux as IR flux. The SEDs also show a range of obscuration from the heavily obscured galaxies (e.g., NGC 3690) to relatively unobscured galaxies (e.g., NGC 4618), or ones with extended UV disks such as NGC 3430 or UGC 6016. 

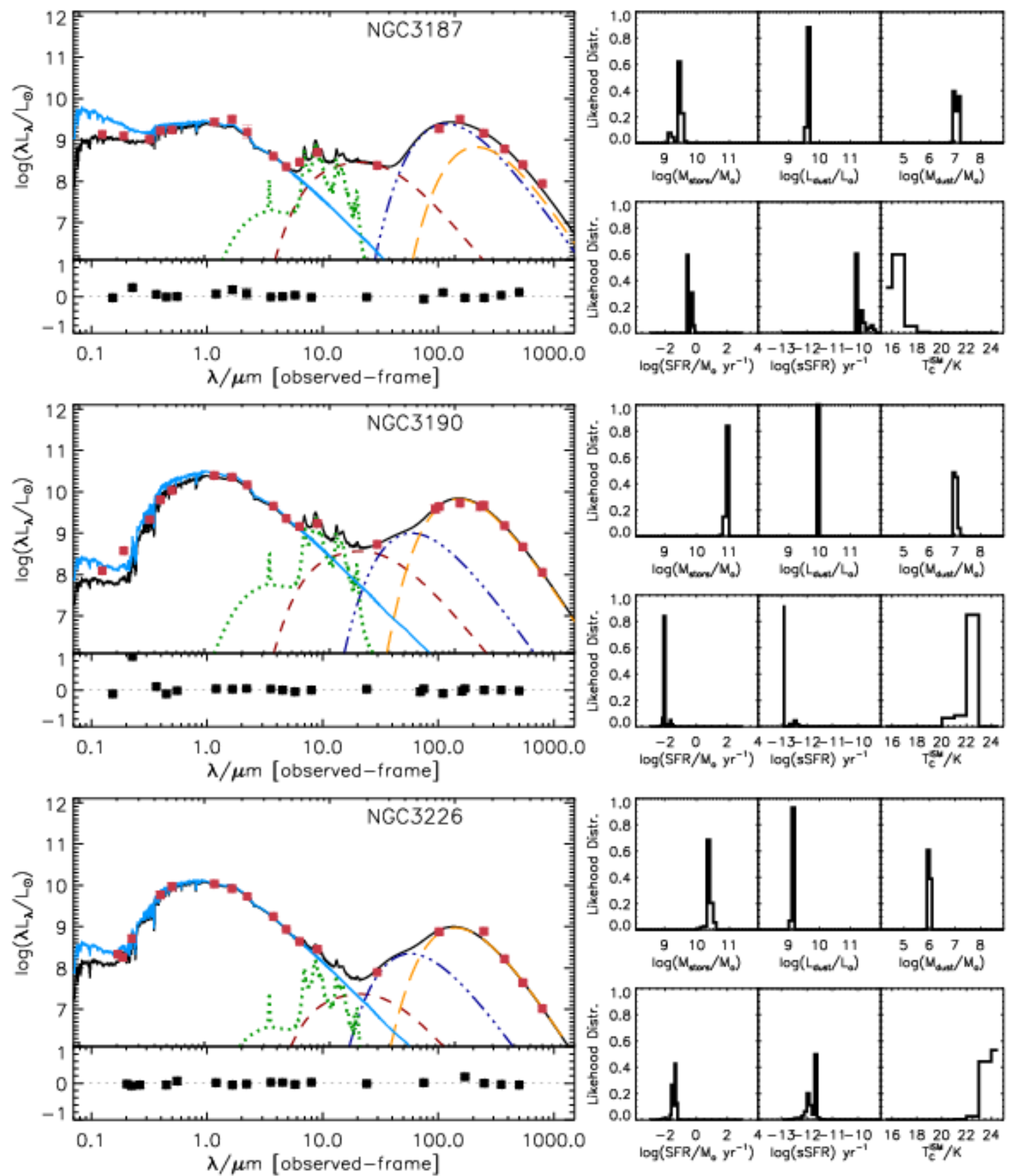

FIG. 9.- As Figure 7, but for NGC 3187 (top), NGC 3190 (middle), and NGC 3226 (bottom).

Appendix C briefly describes each galaxy, discussing any particular issues regarding the photometry and the SED fitting. Note in particular that fits to edge-on galaxies tend to over-estimate the amount of UV obscuration and hence the model UV fluxes tend to be too low compared to the observations.

\section{DISCUSSION}

\subsection{Variation in SED Shape with Interaction Stage}

We now discuss the shape of the SED as a function of the interaction stage. We first normalized each SED to its 2MASS $K s$ luminosity. Emission in the 2MASS $K s$ filter is dominated by the old stellar populations and hence is a good proxy for stellar mass. The comparison between the SEDs is shown in Figure 17. In the inset, we show the median normalized SED for each class of galaxies. The SED shapes between the three stages vary by approximately as much as the variations within a stage. However, there are some significant variations, especially 

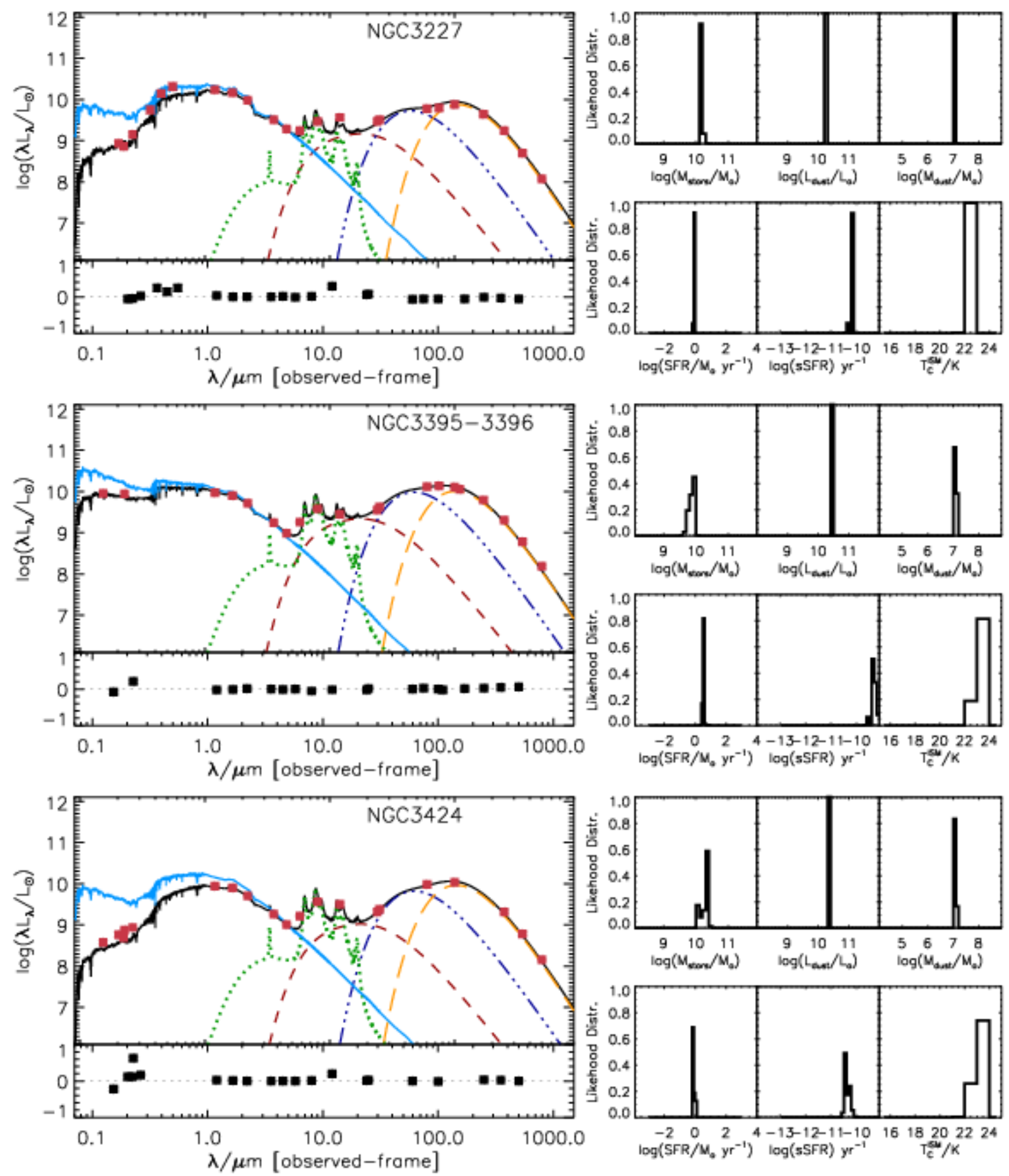

FIG. 10.- As Figure 7, but for NGC 3227 (top), NGC 3395/3396 (middle), and NGC 3424 (bottom).

in the Stage 4 SEDs compared to the Stage 2 and 3 SEDs. Stage 4 galaxies typically have more emission from the hot/warm dust than earlier interaction stages, as evidenced by the stronger $10-60 \mu \mathrm{m}$ emission relative to their stellar mass. Further, Stage 4 galaxies tend to have more warm dust relative to their cold dust FIR emission. In contrast to this variation in the relative MIR emission, all three stages have similar ratios of NIR stellar emission to FIR emission from cold dust. We will discuss the statistical significance of these results in the next section.
These differences in the SEDs are consistent with the results of simulations, which predict that an integrated SED of an interacting system becomes hotter at merger coalescence during the peak of starburst and AGN activity (e.g., Hayward et al. 2011, 2012a; Younger et al. 2009; Narayanan et al. 2010a, 2010b). However, the increase in temperature in this sample of galaxies is unlikely to be driven purely by AGN activity. None of the galaxies in our sample have typical AGN colors, based on their location outside the region in IRAC color space defined 

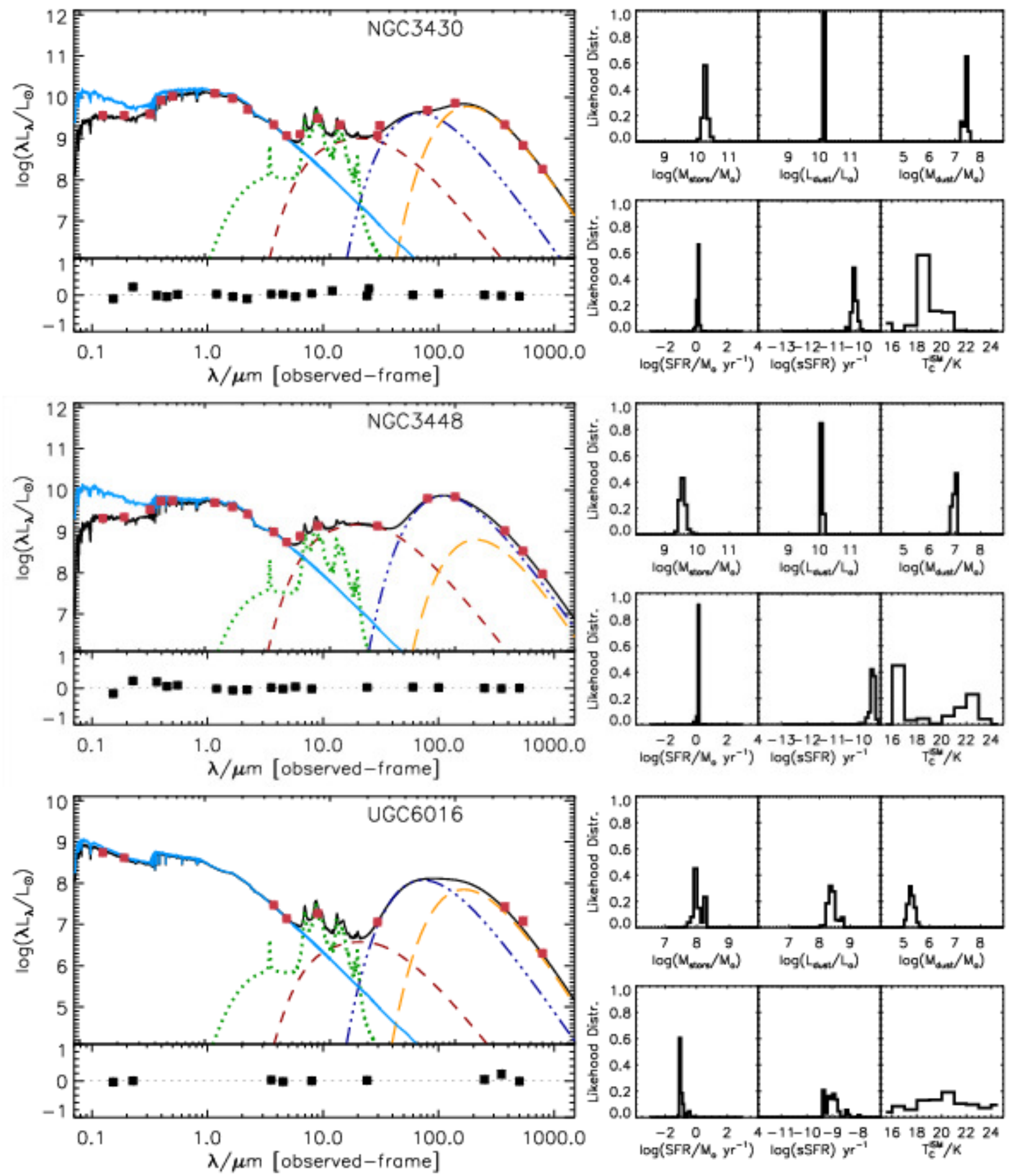

FIG. 11.- As Figure 7, but for NGC 3430 (top), NGC 3448 (middle), and UGC 6016 (bottom). Note that the axes of the UGC 6016 plots have smaller values than the rest of the plots.

by Stern et al. (2005) (Figure 18). Further, while five of the sample galaxies are classified as Seyfert galaxies and three are classified as low-ionization nuclear emissionline region (LINER) galaxies, they are found in all three stages. We used the software DECOMPIR ${ }^{19}$ (Mullaney et al. 2011) to estimate the AGN contribution to the $8-1000 \mu \mathrm{m}$ and $8-35 \mu \mathrm{m}$ emission for these nine galax-

19 http:sites.google.com/site/decompir ies based on the 8-500 $\mu \mathrm{m}$ photometry. We give the individual contributions in the descriptions in the Appendix C; the range of the contribution to the total IR is up to $10 \%$ with some larger, more uncertain values up to $25 \%$. Further, we do not find significant differences in the AGN contribution to either the total infrared luminosity or mid-infrared luminosity, where the Stage 4 SEDs are typically brighter, between the stages. Hence, the effect of AGN on the SEDs of the sample galaxies is 

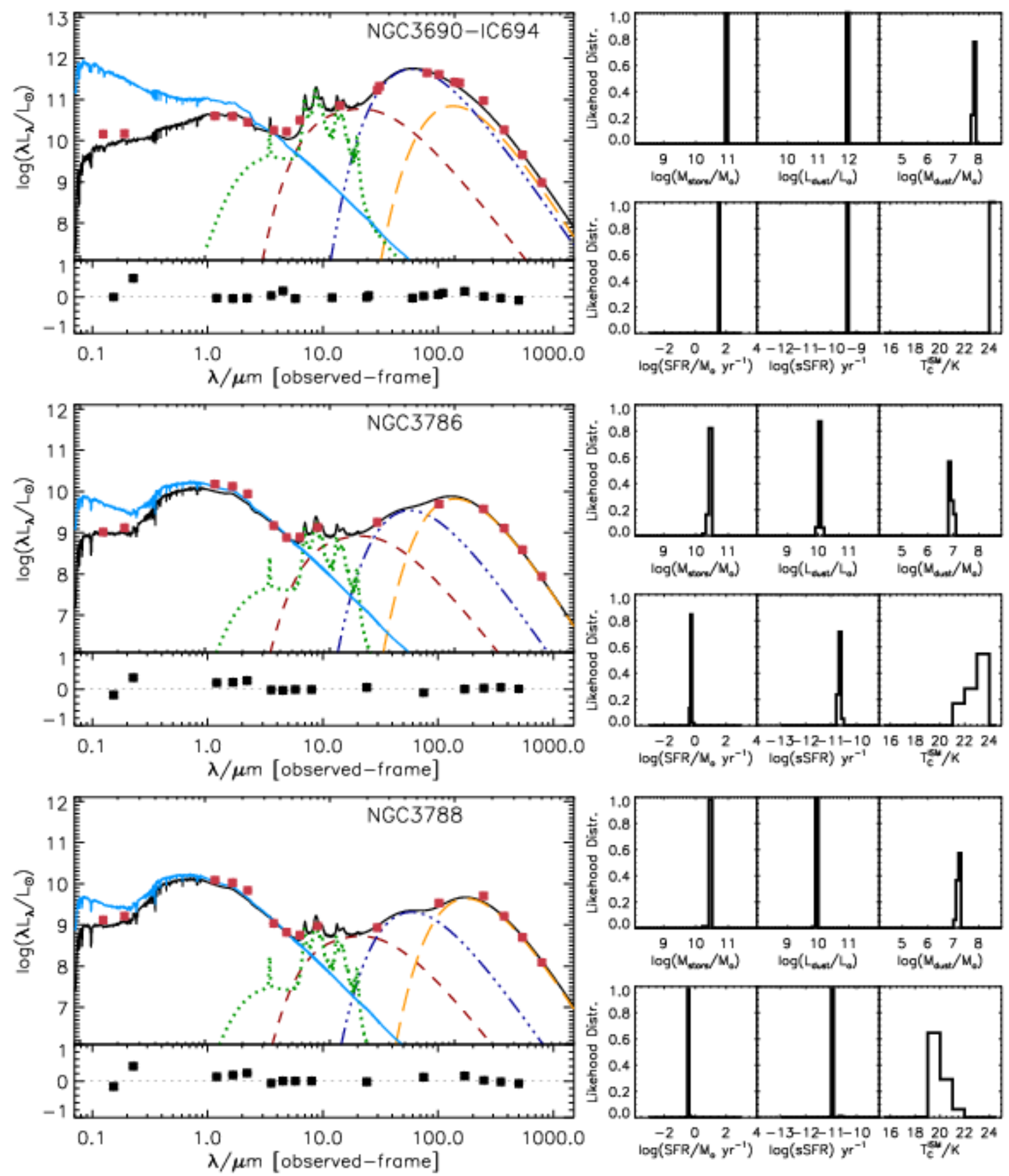

Fig. 12.- As Figure 7, but for NGC 3690/IC 694 (top), NGC 3786 (middle), and NGC 3788 (bottom). Note that the axes of the NGC 3690 plots have larger values than the rest of the plots.

modest and does not affect our conclusions.

The more strongly interacting systems demonstrate a tendency to have, on average, younger stellar populations, resulting in stronger UV emission relative to their NIR emission. Since the UV emission has only been corrected for Milky Way extinction, additional intrinsic extinction could increase this effect. Ignoring NGC 4649, a large elliptical that has very little MIR-FIR emission, the UV bands reflect this tendency and show a large amount of variation between galaxies, which is likely due to the different amounts of dust attenuation and the sensitivity of the UV to recent star formation history. Comparing to the stellar mass proxy of 2 MASS $K s$, Stage 4 galaxies typically have a 1:2 luminosity ratio between emission in the GALEX bands and 2MASS $K s$ band, whereas Stage 2 galaxies typically have a 1:10 luminosity ratio. 

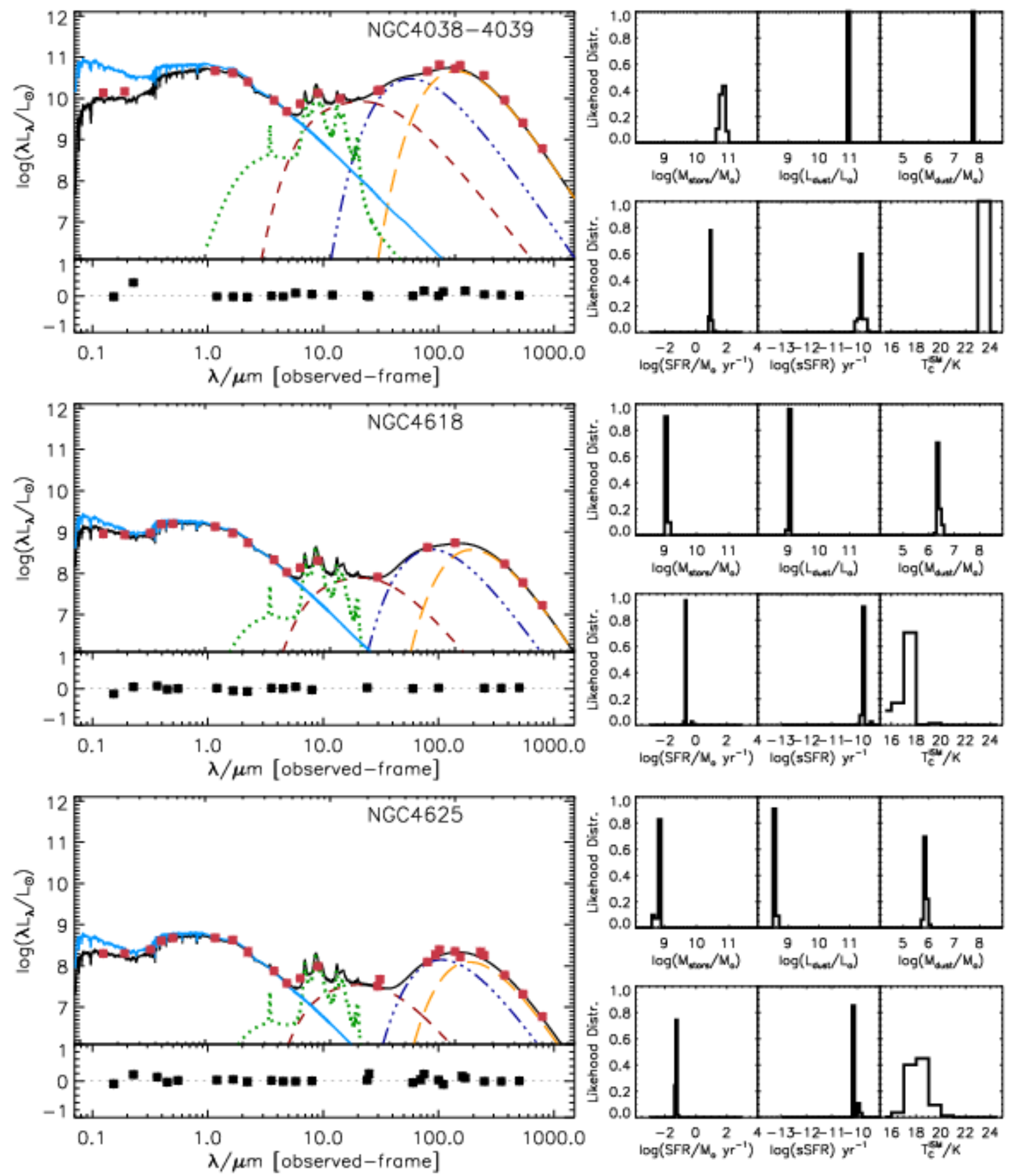

FIG. 13.- As Figure 7, but for NGC 4038/4039 (top), NGC 4618 (middle), and NGC 4625 (bottom).

\subsection{Variations in Galaxy Parameters with Interaction Stage}

Figures 19-20 show the histograms and cumulative distributions for the three interaction stages defined in $\$ 2.2$ for four parameters whose PDFs are shown in Figures 7-16 compared to the non-interacting galaxies. Table 11 gives the results of the Kolmogorov-Smirnov (K-S; e.g., Press et al. 2007) tests performed to determine the likelihood that the samples for the different stages originate from a common parent population for each parameter. With the number of galaxies in our sample, we do not have the same statistical power that the full SIGS sample will have (Brassington et al. 2013, in prep.).

The mass of dust is one of four parameters derived from the SED that differs with marginal statistical significance between the Stage 3 and Stage 4 galaxies $(p=0.02)$. Both the mass of warm dust and of cold dust likewise differ. The warm dust mass also differs between the Stage 4 galaxies and the non-interacting galaxies. In 

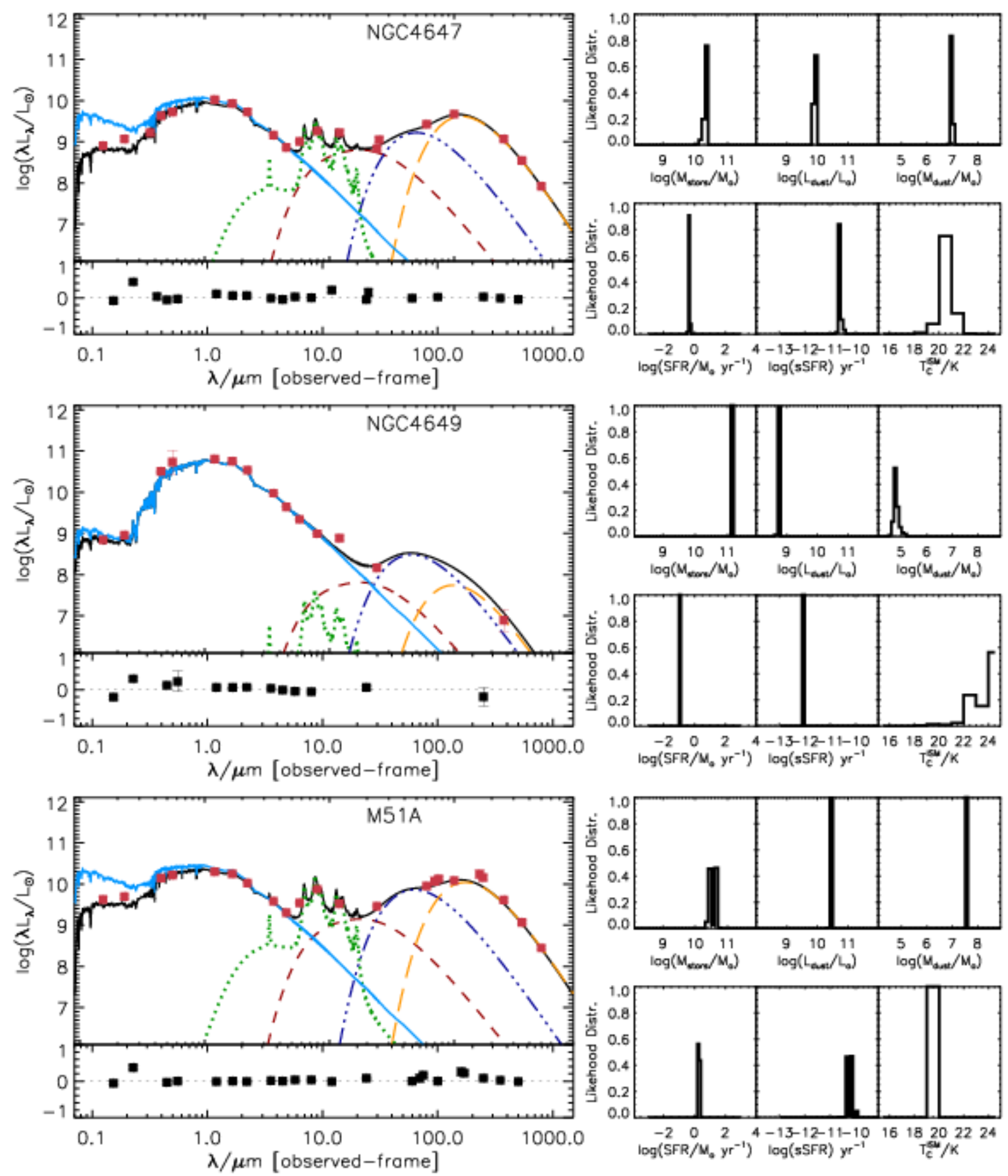

FIG. 14.- As Figure 7, but for NGC 4647 (top), NGC 4649 (middle), and M51A (bottom).

contrast, the distribution of dust mass in the ISM of non-interacting galaxies falls between the distributions of Stage $2 / 3$ and Stage 4 galaxies. The dust mass in the ISM and stellar mass also each show only small increases in their median values as the interaction sequence progresses, and their cumulative distributions are not significantly different. Indeed, both the stellar mass $(p=0.96)$ and total dust mass $(p=0.96)$ for Stage 2 and Stage 3 galaxies are consistent with coming from the same populations. These trends are consistent with the SED shapes described in $\S 5.1$, where the Stage 4 SEDs typically show more emission from the hot/warm dust relative to the cold dust emission and stellar emission.

The dust luminosity (Figure 19, left) shows marginally statistically significant differences between Stage 3 and Stage 4 galaxies $(p=0.01)$ and between the noninteracting galaxies and the Stage 4 galaxies $(p=0.02)$. The median dust luminosity increases with interaction stage by over an order of magnitude between Stage 2 and Stage 4. Elbaz et al. (2011) defined an "IR main 

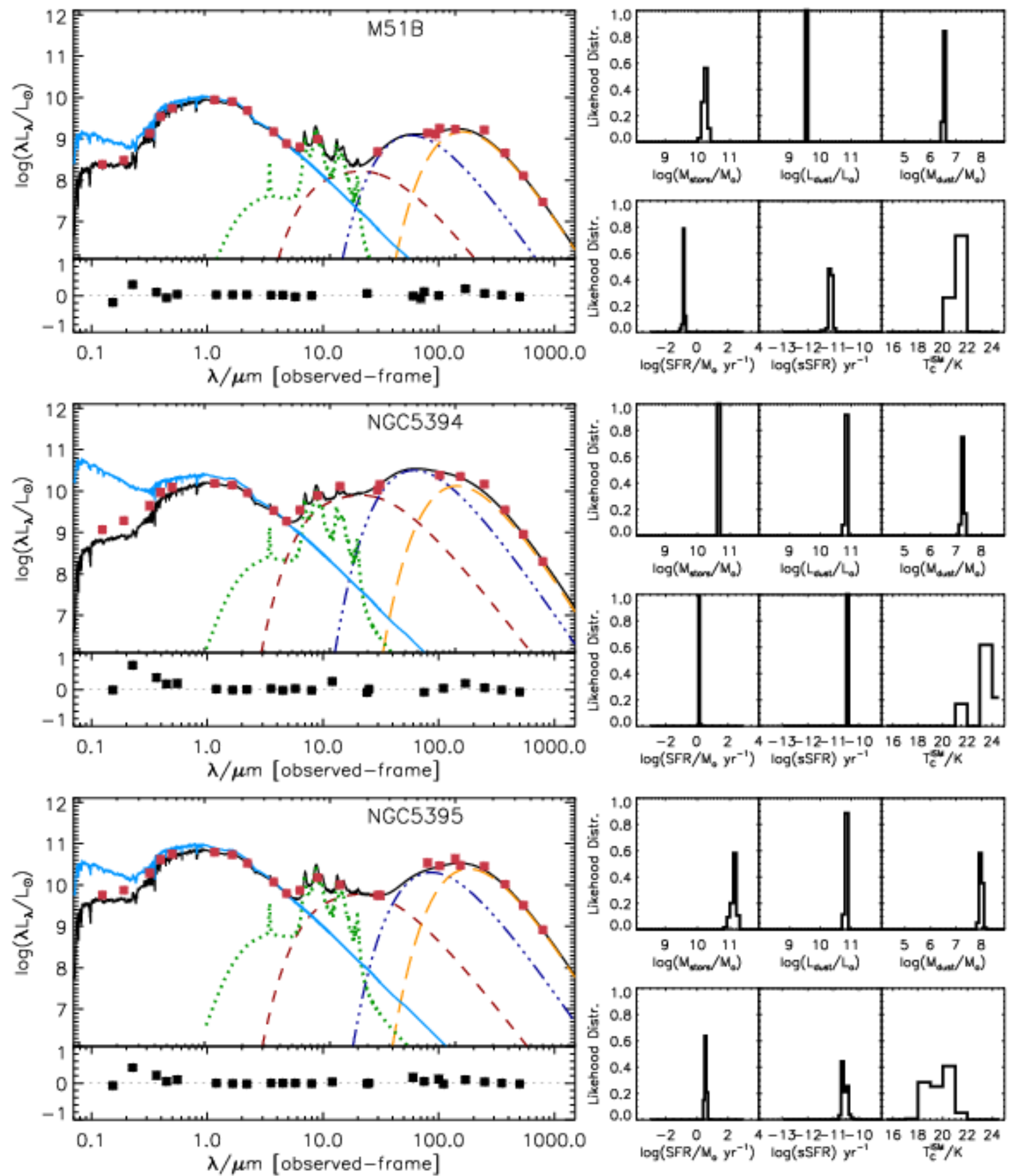

Fig. 15.- As Figure 7, but for M51B (top), NGC 5394 (middle) and NGC 5395 (bottom).

sequence" of galaxies in which the ratio of total IR luminosity to $8 \mu \mathrm{m}$ luminosity has a Gaussian distribution. We examined this ratio for our sample and found good agreement with the expected distribution. The only interacting galaxy that lies off this relation, by roughly an order of magnitude, is the large elliptical NGC 4649, as would be expected. Elbaz et al. (2011) also defined two modes of star formation: a "normal" mode exemplified by the galaxies on the IR main sequence and a "starburst" mode with excess sSFR in comparison. Our sample's agreement with the IR main sequence indicates that our set of interacting galaxies do not contain systems with significantly increased sSFR.

We also considered the evolution of the cold and warm dust temperatures. The cold dust temperature (Figure 19 , right) is the third parameter showing evidence for differences between the Stage 3 and Stage $4(p=0.01)$ and between the non-interacting galaxies and the Stage 4 galaxies $(p=0.01)$. The cold dust temperature's median value varies in a similar manner to the IR luminosity, in- 

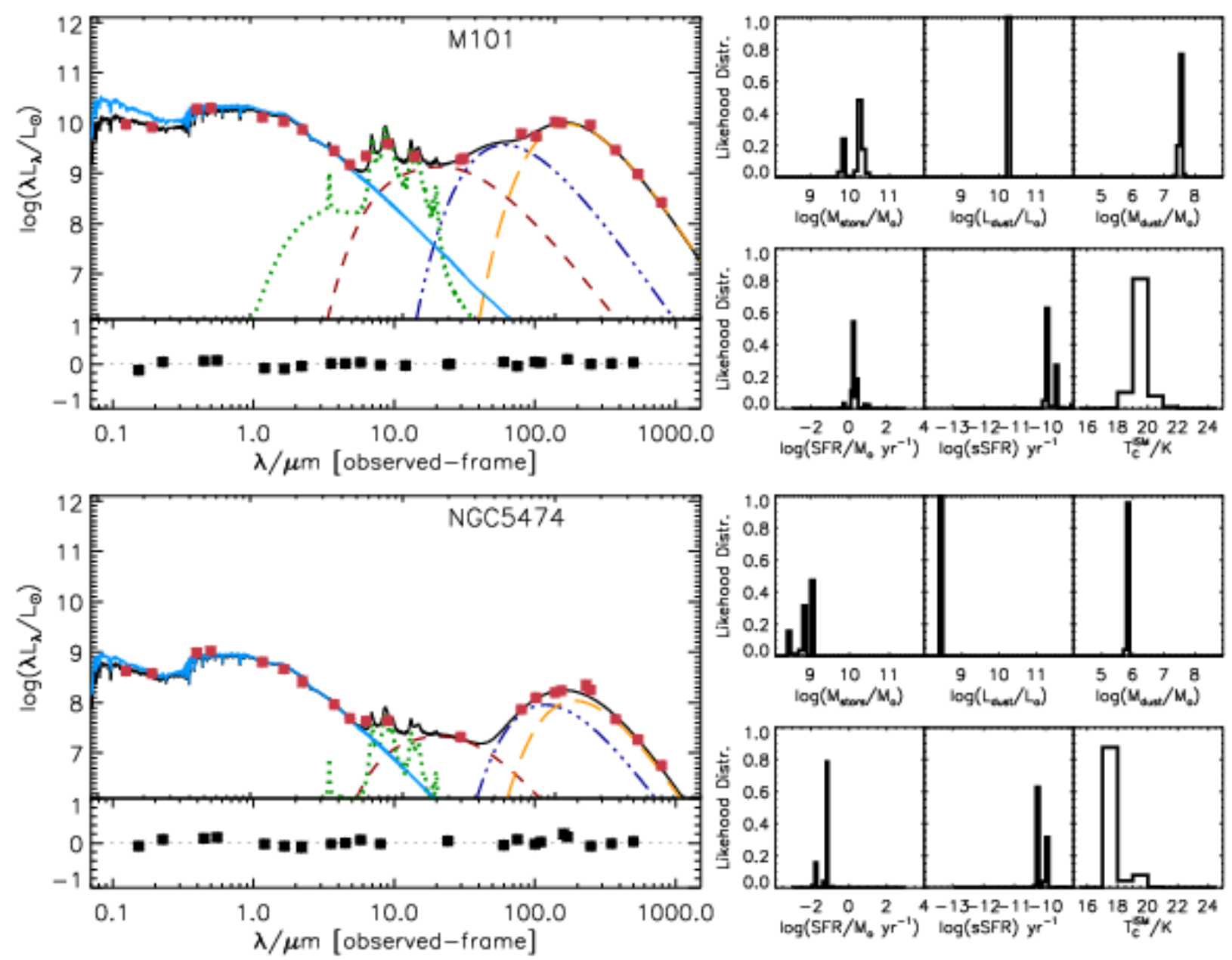

FIG. 16.- As Figure 7, but for M101 (top) and NGC 5474 (bottom).

creasing between Stages 3 and 4 but relatively constant between Stages 2 and 3 ; the median value of the warm dust temperature is by contrast fairly constant. Only Stage 4 does not span the range of the $15-25 \mathrm{~K}$ cold dust temperature, while in Stage 2 and Stage 4 the warm dust temperatures are confined to the $45-60 \mathrm{~K}$ range. The similarity in evolutionary trend in the IR luminosity and cold dust temperature is likely due to the predominance of cold dust mass and luminosity in the total dust estimates. We might expect a similar trend to be exhibited in the temperature of the warm dust primarily present in the stellar birth clouds, however the warm dust temperature is less well constrained in MAGPHYS than the cold dust temperature. The cold dust contribution and warm dust temperature are both correlated with the warm dust mass (which drives the MIR continuum intensity and the shape of the SED). Therefore, the warm dust temperature PDFs tends to be broader, with a $68 \%$ range that is typically four to five times the size of the cold dust $68 \%$ confidence interval. However, since the cold dust mass is typically over $\sim 80 \%$ of the total dust mass, the total dust mass is still fairly well constrained.

The SFR, shown in Figure 20 (left), shows an increase in median value with interaction stage, like the the dust mass and dust luminosity, and has a marginally significant probability the same population did not yield the Stage 4 galaxies as well as the Stage $2(p=0.03)$, Stage $3(p=0.02)$, and non-interacting $(p=0.02)$ galaxies. Since the warm $(30-60 \mathrm{~K})$ dust, primarily heated by young stars with ages less than $10 \mathrm{Myr}$, contributes significantly to the total dust luminosity, it makes sense that these three parameters show similar evolution over the interaction stages. However, an increase in SFR between the different stages could be also attributed to our Stage 4 galaxies simply being larger with greater gas reservoirs. To test this, we also examined the evolution of sSFR over the interaction stages (Figure 20, right). In contrast to SFR, we do not find much difference in the median values of the sSFR, and the cumulative distributions are very similar in both width and normalization. We therefore do not see enhanced sSFR in more evolved mergers, consistent with the ratios of total IR luminosity to $8 \mu \mathrm{m}$ luminosity being due to a "normal" mode of star formation for our whole sample. We also do not see differences between the distributions of the sSFR of the three interacting galaxy samples and of the non-interacting galaxies. $\mathrm{Xu}$ et al. (2010) and Yuan et al. (2012) examined star formation in a sample of local major mergers and both found that that the sSFR distributions of galaxies in spiralspiral interactions and non-interacting systems were unlikely to originate from the same population (based on a $\mathrm{K}-\mathrm{S}$ test: $p=0.03-0.04)$. However, they also found a mass dependence in the enhancement of sSFR; only those galaxies with $M_{*}>10^{10.5} M_{\odot}$ were found to have signif- 
TABLE 10

MAGPHYS PARAMETERS

\begin{tabular}{|c|c|c|c|c|c|c|c|c|c|}
\hline Name & $\begin{array}{l}\text { Dust Luminosity } \\
\quad\left(10^{9} \mathrm{~L}_{\odot}\right)\end{array}$ & $\begin{array}{c}\text { Stellar Mass } \\
\left(10^{10} \mathrm{M}_{\odot}\right)\end{array}$ & $\begin{array}{r}\text { Dust } \\
\left(10^{7}\right.\end{array}$ & $\begin{array}{l}\text { Mass } \\
\text { M } \odot))\end{array}$ & $\begin{array}{r}\mathrm{SF} \\
\left(\mathrm{M}_{\odot}\right.\end{array}$ & $\begin{array}{l}\mathrm{R}^{\mathrm{a}} \\
\left.\mathrm{yr}^{-1}\right)\end{array}$ & $\begin{array}{l}\text { Specific SFR } \\
\left(10^{-11} \mathrm{yr}^{-1}\right)\end{array}$ & $\begin{array}{l}\text { Cold Dust Temp. } \\
(\mathrm{K})\end{array}$ & $\begin{array}{l}\text { Warm Dust Temp. } \\
(\mathrm{K})\end{array}$ \\
\hline NGC2976 & $0.86_{-0.02}^{+0.01}$ & $0.10 \stackrel{+0.05}{+0.01}$ & 0.17 & $\pm_{-1}$ & 0.084 & $\begin{array}{l}+0.009 \\
+0.015\end{array}$ & $8.51{ }_{-3.14}^{+0.20}$ & $18.8_{-0.2}^{+0.1}$ & $48.6_{-0.1}^{+3.7}$ \\
\hline NGC3031 & $4.05_{-0.05}^{+0.05}$ & $2.95 \quad{ }_{-0.07}^{+2.41}$ & 1.18 & & 0.373 & & $1.35{ }_{-0.59}^{+0.03}$ & $18.4_{-0.9}^{+0.6}$ & $53.6_{-21.2}^{+3.5}$ \\
\hline NGC3034 & $54.95_{-1.25}^{+1.28}$ & $1.45{ }_{-0.03}^{+0.03}$ & 0.86 & $\begin{array}{l}+0.09 \\
{ }_{-0.18}\end{array}$ & 0.728 & $\begin{array}{l}+0.008 \\
-0.008\end{array}$ & $5.37 \begin{array}{r}+0.13 \\
-0.12\end{array}$ & $23.5_{-0.7}^{+0.8}$ & $51.4_{-8.0}^{+5.6}$ \\
\hline NGC3077 & $0.75_{-0.02}^{+0.02}$ & $0.068_{-0.002}^{+0.035}$ & 0.041 & $\begin{array}{l}-0.18 \\
+0.001 \\
-0.001\end{array}$ & 0.097 & $\begin{array}{l}-0.008 \\
+0.031 \\
-0.011\end{array}$ & $14.96{ }_{-8.20}^{+6.17}$ & $22.9_{-0.1}^{+0.1}$ & $52.5_{-0.1}^{+0.0}$ \\
\hline NGC3185 & $3.29_{-0.04}^{+0.04}$ & $1.45{ }_{-0.03}^{+0.03}$ & 0.49 & & 0.183 & & $1.20{ }_{-0.03}^{+0.03}$ & $20.2_{-0.4}^{+0.4}$ & $57.8_{-3.7}^{+1.4}$ \\
\hline NGC3187 & $4.09_{-0.05}^{+0.05}$ & $0.31{ }_{-0.04}^{+0.03}$ & 1.02 & $\begin{array}{l}-0.07 \\
{ }_{-0.05}^{+0.06}\end{array}$ & 0.274 & $\begin{array}{l}-0.002 \\
+0.325 \\
-0.003\end{array}$ & $8.51_{-0.11}^{+10.033}$ & $16.2_{-0.3}^{+0.4}$ & $32.6_{-1.7}^{+9.7}$ \\
\hline NGC3190 & $8.55_{-0.10}^{+0.82}$ & 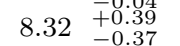 & 1.44 & $\begin{array}{l}-0.03 \\
{ }_{-0}^{+0.03}\end{array}$ & 0.0084 & $\begin{array}{l}-0.0004 \\
+0.0004 \\
-0.0004\end{array}$ & $0.011_{-0.001}^{+0.001}$ & $22.3_{-0.1}^{+0.3}$ & $57.9_{-0.2}^{+0.1}$ \\
\hline NGC3226 & $1.40_{-0.11}^{+0.10}$ & $2.29{ }_{-0.15}^{+0.28}$ & 0.10 & $\begin{array}{l}+0.01 \\
{ }_{-0.01}^{+0.01}\end{array}$ & 0.045 & $\begin{array}{l}+0.003 \\
+0.017\end{array}$ & $2.14 \stackrel{-0.94}{+0.05}_{-0.94}$ & $24.1_{-0.5}^{+0.7}$ & $54.4_{-3.7}^{+4.0}$ \\
\hline NGC3227 & $16.98_{-0.39}^{+0.11}$ & $1.32 \quad \begin{array}{l}-0.03 \\
-0.03\end{array}$ & 1.15 & $\begin{array}{l}-0.01 \\
{ }_{-0.03}^{+0.03}\end{array}$ & 0.836 & $\begin{array}{l}-0.017 \\
+0.019 \\
-0.019\end{array}$ & $6.76{ }_{-0.15}^{+0.94}$ & $22.5_{-0.1}^{+0.5}$ & $59.1_{-0.1}^{+0.1}$ \\
\hline NGC3395/3396 & $26.30_{-0.60}^{+0.61}$ & $0.70 \begin{array}{l}+0.11 \\
{ }_{-0.08}\end{array}$ & 1.13 & $\begin{array}{r}+0.39 \\
{ }_{-0.01}^{+0.39}\end{array}$ & 3.289 & $\begin{array}{l}+0.235 \\
+0.148\end{array}$ & $42.17_{-0.96}^{+10.92}$ & $23.8_{-1.3}^{+0.1}$ & $55.8_{-0.1}^{+3.1}$ \\
\hline NGC3424 & $20.42_{-0.46}^{+0.48}$ & $2.04{ }_{-0.81}^{+0.05}$ & 1.14 & $\begin{array}{l}+0.17 \\
{ }_{-0.03}\end{array}$ & 0.753 & $\begin{array}{l}+0.163 \\
+0.050\end{array}$ & $3.80 \quad \begin{array}{l}+2.22 \\
-0.09\end{array}$ & $23.3_{-0.7}^{+0.1}$ & $55.4_{-4.6}^{+0.1}$ \\
\hline NGC3430 & $13.49_{-0.61}^{+0.46}$ & $1.82 \quad \begin{array}{l}-0.81 \\
+0.23\end{array}$ & 2.55 & $\begin{array}{l}-0.03 \\
{ }_{-0.35}^{+0.12}\end{array}$ & 1.355 & $\begin{array}{l}-0.050 \\
+0.165 \\
-0.188\end{array}$ & $7.59 \begin{array}{l}-0.09 \\
+2.96\end{array}$ & $19.0_{-0.8}^{+0.7}$ & $55.5_{-5.1}^{+3.6}$ \\
\hline NGC3448 & $11.22_{-0.26}^{+1.37}$ & $0.35{ }_{-0.05}^{+0.07}$ & 0.91 & $\begin{array}{l}+0.12 \\
{ }_{-0.16}\end{array}$ & 1.371 & $\begin{array}{l}+0.065 \\
+0.047\end{array}$ & $37.58+9.73$ & $18.7_{-2.3}^{+3.9}$ & $32.9_{-0.4}^{+22.2}$ \\
\hline UGC6016 & $\begin{array}{l}0.25_{-0.08}^{+0.09} \\
\end{array}$ & $0.010_{-0.002}^{+0.009}$ & 0.020 & $\begin{array}{l}-0.007 \\
-0.005\end{array}$ & 0.091 & $\begin{array}{l}-0.048 \\
+0.008 \\
-0.008\end{array}$ & $94.41_{-47.09}^{+55.22}$ & $20.4_{-2.7}^{+2.8}$ & $41.1_{-7.2}^{+6.4}$ \\
\hline NGC3690/IC694 & $812.8_{-18.50}^{+18.93}$ & $8.13{ }_{-0.19}^{+0.19}$ & 7.28 & $\begin{array}{l}-0.005 \\
+1.176 \\
-1.56\end{array}$ & 35.65 & $\begin{array}{l}-0.008 \\
+0.83 \\
-0.81\end{array}$ & $42.17 \stackrel{+}{+}+0.98$ & $24.8_{-0.1}^{+0.2}$ & $58.0_{-4.3}^{+0.1}$ \\
\hline NGC3786 & $12.59_{-1.87}^{+0.29}$ & $3.02 \quad{ }_{-0.62}^{+0.19}$ & 0.72 & $\begin{array}{l}+1.29 \\
+0.02\end{array}$ & 0.612 & $\begin{array}{l}+0.014 \\
+0.109\end{array}$ & $2.14{ }_{-0.23}^{+0.05}$ & $23.8_{-2.3}^{+0.1}$ & $59.6_{-1.0}^{+0.4}$ \\
\hline NGC3788 & $8.07_{-0.09}^{+0.19}$ & $2.95 \quad \begin{array}{l}+0.07 \\
+0.07\end{array}$ & 1.59 & $\begin{array}{l}+0.04 \\
{ }_{-0.27}^{0.04}\end{array}$ & 0.352 & $\begin{array}{l}+0.008 \\
+0.004\end{array}$ & $1.20{ }_{-0.03}^{+0.03}$ & $19.6_{-0.1}^{+0.7}$ & $58.2_{-2.0}^{+0.9}$ \\
\hline NGC4038/4039 & $97.72_{-2.22}^{+2.28}$ & $6.46{ }_{-1.33}^{+0.02}$ & 5.15 & $\begin{array}{l}-0.21 \\
{ }_{-0.12}^{+0.12}\end{array}$ & 8.954 & $\begin{array}{l}-0.004 \\
+0.422 \\
-0.788\end{array}$ & $13.34 \quad+3.45$ & $23.7_{-0.1}^{+0.1}$ & $59.9_{-0.1}^{+0.0}$ \\
\hline NGC4618 & $1.04_{-0.01}^{+0.02}$ & $0.11{ }_{-0.01}^{+0.01}$ & 0.25 & $\begin{array}{l}+0.04 \\
{ }_{-0.01}^{+0.04}\end{array}$ & 0.225 & $\begin{array}{l}+0.003 \\
+0.003\end{array}$ & $18.84+0.44$ & $17.7_{-1.6}^{+0.1}$ & $40.8_{-7.3}^{+0.1}$ \\
\hline NGC4625 & $0.39_{-0.01}^{+0.01}$ & $0.063_{-0.001}^{+0.001}$ & 0.074 & $\begin{array}{l}-0.01 \\
+0.007 \\
-0.004\end{array}$ & 0.050 & $\begin{array}{l}-0.003 \\
+0.001 \\
{ }_{-0}\end{array}$ & $7.59 \begin{array}{l}{ }_{-0.17}^{+0.18} \\
-0.17\end{array}$ & $18.4_{-1.3}^{+0.0}$ & $33.7_{-2.8}^{+3.3}$ \\
\hline NGC4647 & $8.07_{-0.27}^{+0.19}$ & $2.04{ }_{-0.09}^{+0.05}$ & 0.99 & $\begin{array}{l}-0.04 \\
+0.02 \\
-0.04\end{array}$ & 0.470 & $\begin{array}{l}-0.001 \\
+0.011 \\
-0.011\end{array}$ & $2.40{ }_{-0.05}^{+0.17}$ & $20.9_{-0.1}^{+0.3}$ & $52.7_{-0.1}^{+4.8}$ \\
\hline NGC4649 & $0.50_{-0.01}^{+0.01}$ & $13.80_{-0.31}^{+0.32}$ & 0.006 & $\begin{array}{l}+0.002 \\
-0.001 \\
-0.002\end{array}$ & 0.114 & $\begin{array}{l}-0.011 \\
+0.001 \\
{ }_{-0}\end{array}$ & $0.085_{-0.002}^{+0.002}$ & $24.5_{-1.9}^{+0.1}$ & $59.5_{-1.6}^{+0.4}$ \\
\hline M51A & $25.70_{-0.59}^{+0.61}$ & $3.02{ }_{-0.33}^{+0.77}$ & 3.78 & $\begin{array}{l}-0.01 \\
+0.09 \\
-0.09\end{array}$ & 1.936 & $\begin{array}{l}-0.001 \\
+0.138 \\
-0.022\end{array}$ & $6.76 \stackrel{+0.82}{+2.50}$ & $19.6_{-0.1}^{+0.9}$ & $54.9_{-0.1}^{+0.0}$ \\
\hline M51B & $3.65_{-0.20}^{+0.08}$ & $1.95{ }_{-0.36}^{+0.05}$ & 0.34 & $\begin{array}{l}+0.03 \\
{ }_{-0.02}\end{array}$ & 0.142 & $\begin{array}{l}+0.003 \\
-0.014\end{array}$ & $0.76{ }_{-0.02}^{+0.50}$ & $21.0_{-0.3}^{+0.1}$ & $59.3_{-5.8}^{+0.1}$ \\
\hline NGC5394 & $63.10_{-1.44}^{+1.47}$ & $4.79{ }_{-0.11}^{+0.11}$ & 1.77 & $\begin{array}{l}+0.26 \\
{ }_{-0.08}\end{array}$ & 1.538 & $\begin{array}{l}-0.018 \\
+0.018 \\
-0.035\end{array}$ & $3.39{ }_{-0.08}^{+0.08}$ & $23.2_{-1.5}^{+1.0}$ & $55.6_{-2.2}^{+1.8}$ \\
\hline NGC5395 & $66.07_{-1.50}^{+4.44}$ & $15.14_{-4.42}^{+0.71}$ & 9.48 & $\begin{array}{l}-0.08 \\
+1.66 \\
-1.22\end{array}$ & 3.690 & $\begin{array}{l}-0.0303 \\
+0.513\end{array}$ & $2.69{ }_{-0.55}^{+1.08}$ & $19.7_{-0.8}^{+0.5}$ & $45.3_{-9.7}^{+5.6}$ \\
\hline M101 & $18.20_{-0.82}^{+0.42}$ & $1.82 \quad \begin{array}{l}-4.47 \\
-0.12\end{array}$ & 3.25 & $\begin{array}{l}-1.22 \\
-0.31 \\
-0.11\end{array}$ & 1.959 & $\begin{array}{l}-0.513 \\
+0.905 \\
-0.367\end{array}$ & $11.89_{-1.29}^{+9.55}$ & $19.4_{-0.4}^{+0.8}$ & $58.2_{-2.2}^{+0.8}$ \\
\hline NGC5474 & $0.26_{-0.01}^{+0.01}$ & $0.074_{-0.041}^{+0.041}$ & 0.072 & $\begin{array}{l}-0.11 \\
+0.002 \\
-0.002\end{array}$ & 0.070 & $\begin{array}{l}-0.007 \\
+0.007 \\
-0.054\end{array}$ & $6.03{ }_{-0.14}^{+4.59}$ & $17.9_{-0.1}^{+0.4}$ & $30.4_{-0.1}^{+0.8}$ \\
\hline NGC925 & $4.39_{-0.10}^{+0.10}$ & $0.92{ }_{-0.52}^{+0.02}$ & 2.38 & $\begin{array}{l}{ }_{-0.08}^{+0.03} \\
-0.08\end{array}$ & 0.459 & $\begin{array}{l}+0.236 \\
{ }_{-0.010}\end{array}$ & $4.79{ }_{-0.11}^{+7.10}$ & $15.4_{-0.1}^{+0.7}$ & $35.7_{-4.9}^{+6.2}$ \\
\hline NGC1291 & $2.17_{-0.02}^{+0.05}$ & $6.17 \quad{ }_{-0.54}^{+0.75}$ & 0.89 & $\begin{array}{l}+0.14 \\
{ }_{-0.03}^{+0.14}\end{array}$ & 0.118 & $\begin{array}{r}+0.014 \\
+0.020\end{array}$ & $0.13{ }_{-0.06}^{+0.08}$ & $17.2_{-0.4}^{+0.1}$ & $57.4_{-1.9}^{+1.9}$ \\
\hline NGC2841 & $4.54_{-0.10}^{+0.11}$ & $2.95_{-0.32}^{+1.41}$ & 1.98 & $\begin{array}{l}-0.02 \\
{ }_{-0.15}^{+0.32}\end{array}$ & 0.220 & $\begin{array}{l}+0.044 \\
+0.069\end{array}$ & $0.54{ }_{-0.22}^{+0.09}$ & $17.2_{-0.4}^{+0.4^{+}}$ & $55.0_{-1.3}^{+1.9}$ \\
\hline NGC3049 & $4.29_{-0.19}^{+0.10}$ & $0.11{ }_{-0.01}^{+0.01}$ & 0.66 & $\begin{array}{l}-0.19 \\
{ }_{-0.14}^{+0.07}\end{array}$ & 0.459 & $\begin{array}{l}+0.569 \\
+0.036\end{array}$ & $37.58+63.76$ & $17.3_{-0.3}^{+1.4}$ & $54.9_{-2.5}^{+1.3}$ \\
\hline NGC3184 & $4.86_{-0.11}^{+0.11}$ & $\begin{array}{ll}0.42 \quad{ }_{-0.07}^{+0.09} \\
\end{array}$ & 1.25 & & 0.207 & $\begin{array}{l}+0.216 \\
-0.087\end{array}$ & $2.69 \begin{array}{l}+7.52 \\
+7.57\end{array}$ & $18.2_{-0.7}^{+0.3}$ & $52.9_{-4.3}^{+3.3}$ \\
\hline NGC3521 & $21.88_{-0.50}^{+0.51}$ & $4.57_{-1.10}^{+1.05}$ & 3.52 & $\begin{array}{l}+0.43 \\
-0.56\end{array}$ & 0.771 & $\begin{array}{l}-0.004 \\
+0.130\end{array}$ & $1.35{ }_{-0.56}^{+0.41}$ & $19.9_{-0.4}^{+0.5}$ & $50.6_{-4.9}^{+3.3}$ \\
\hline NGC3621 & $6.64_{-0.30}^{+0.15}$ & $0.84{ }_{-0.18}^{+-1.10}$ & 1.29 & $\begin{array}{l}-0.56 \\
{ }_{-0.18}^{+0.16}\end{array}$ & 0.598 & $\begin{array}{l}-0.130 \\
+0.050 \\
-0.059\end{array}$ & $5.37{ }_{-1.39}^{+0.56}$ & $18.6_{-0.6}^{+0.4}$ & $46.2^{-4.9}+{ }^{+5}$ \\
\hline NGC3938 & $7.80_{-0.18}^{+0.18}$ & $0.26{ }_{-0.01}^{+0.05}$ & 1.50 & $\begin{array}{l}+0.13 \\
{ }_{-0.10}^{+0.13}\end{array}$ & 0.225 & $\begin{array}{l}+0.470 \\
-0.005\end{array}$ & $8.51{ }_{-0.19}^{+12.62}$ & $19.1_{-0.4}^{+0.2}$ & $46.5_{-5.6}^{+8.2}$ \\
\hline NGC4236 & $0.27_{-0.01}^{+0.01}$ & $0.020_{-0.001}^{+0.001}$ & 0.009 & $\begin{array}{l}-0.001 \\
-0.001\end{array}$ & 0.022 & $\begin{array}{l}+0.001 \\
+0.001\end{array}$ & $10.59{ }_{-0.24}^{+0.19}$ & $24.6_{-0.6}^{+0.4}$ & $48.4_{-0.1}^{+0.8}$ \\
\hline NGC4559 & $7.36_{-0.25}^{+0.09}$ & $0.52{ }_{-0.01}^{+0.01}$ & 2.00 & $\begin{array}{l}-0 \\
+0 \\
-0\end{array}$ & 1.294 & $\begin{array}{l}-0.001 \\
+0.015 \\
-0.058\end{array}$ & $23.71 \quad \begin{array}{l}-0.54 \\
-0.54\end{array}$ & $17.8_{-1.1}^{+0.6}$ & $45.8_{-8.3}^{+7.1}$ \\
\hline NGC4594 & $7.28_{-0.17}^{+0.17}$ & $32.36_{-0.74}^{+0.75}$ & 2.77 & & 0.032 & & $0.011_{-0.001}^{+0.001}$ & $17.8_{-0.1}^{+0.1}$ & $59.1_{-2.3}^{+0.3}$ \\
\hline NGC4736 & $7.03_{-0.24}^{+0.42}$ & $1.02{ }_{-0.05}^{+0.46}$ & 0.53 & -0.06 & 0.598 & $\begin{array}{l}21 \\
33\end{array}$ & $4.27 \stackrel{+0.14}{+0.76}$ & $21.8_{-1.0}^{+0.1}$ & $35.4_{-3.0}^{+12.7}$ \\
\hline NGC4826 & $4.29_{-0.10}^{+0.10}$ & $3.72 \quad \begin{array}{l}{ }_{-0.27}^{+0.05} \\
\end{array}$ & 0.31 & $\begin{array}{l}-0 \\
+0\end{array}$ & 0.070 & & $0.09{ }_{-0.13}^{-1.76}$ & $23.4_{-0.6}^{+1.0}$ & $\begin{array}{r}58.4_{-3.4}^{+0.0} \\
\text { +0.8 }\end{array}$ \\
\hline NGC5055 & $19.50_{-0.44}^{+0.45}$ & $5.01_{-1.38}^{+0.61}$ & 4.00 & & 0.906 & ${ }_{-0 .}^{+0 .}$ & $1.35{ }_{-0.56}^{+0.49}$ & $19.2_{-0.3}^{+0.0}$ & $54.6_{-6.0}^{+1.8}$ \\
\hline NGC6946 & $23.99_{-0.55}^{+0.56}$ & $2.19{ }_{-0.49}^{+0.27}$ & 2.77 & $\begin{array}{l}+0.34 \\
+0.41\end{array}$ & 1.629 & $\begin{array}{l}+0.241 \\
+0.350\end{array}$ & $6.76 \begin{array}{l}\begin{array}{c}0.90 \\
+0.82\end{array} \\
-0.82\end{array}$ & $20.7_{-0.8}^{+0.3}$ & $54.9_{-1.7}^{+2.0}$ \\
\hline
\end{tabular}

Note. - Median of the PDFs determined by MAGPHYS. The uncertainty given is the $68 \%$ confidence range.

${ }^{a}$ Averaged over $100 \mathrm{Myr}$

icant enhancements. Our sample shows a similar trend, but we only have six galaxies with $M_{*}>10^{10.5} M_{\odot}$ of which one is an elliptical, and they are spread across Stages 3 and 4.

We also examined the star formation efficiency, which we define as the ratio of SFR to warm (30-60 K) birth cloud dust mass, a proxy for molecular gas mass. While the ratio of dust mass to gas mass is not necessarily the same between galaxies, this ratio provides a means of estimating the star formation efficiency under the assumption of constant gas-to-dust ratio. This star formation efficiency allows us to compare the SFR taking into account the variable gas reservoirs. We find that regardless of stage, the star formation efficiency ranges over more than three orders of magnitude and the cumulative distributions show no evidence of originating from different populations. This result agrees with the findings of Casasola et al. (2004) who found similar star formation efficiencies, defined as the ratio of FIR luminosity (a proxy for SFR) and molecular hydrogen mass, for interacting and non-interacting galaxies.

All the apparent variations with interaction stage come 
TABLE 11

K-S Probabilities

\begin{tabular}{lcccccc}
\hline \hline \multirow{2}{*}{ Parameter } & \multicolumn{2}{c}{ With Non-Interacting } & \multicolumn{3}{c}{ Between Stages } \\
& Stage 2 & Stage 3 & Stage 4 & Stages 2 and 3 & Stages 3 and 4 & Stages 2 and 4 \\
\hline Dust Luminosity & 0.238 & 0.224 & $\mathbf{0 . 0 1 7}$ & 0.767 & $\mathbf{0 . 0 0 8}$ & 0.129 \\
Total Dust Mass & 0.095 & 0.072 & 0.462 & 0.964 & $\mathbf{0 . 0 2 2}$ & 0.423 \\
Stellar Mass & 0.366 & 0.655 & 0.569 & 0.964 & 0.271 & 0.129 \\
SFR & 0.980 & 0.983 & $\mathbf{0 . 0 1 7}$ & 0.964 & $\mathbf{0 . 0 2 2}$ & $\mathbf{0 . 0 2 8}$ \\
sSFR & 0.569 & 0.953 & 0.606 & 0.964 & 0.768 & 0.883 \\
Cold Dust Temperature & 0.337 & 0.417 & $\mathbf{0 . 0 0 6}$ & 0.767 & $\mathbf{0 . 0 0 8}$ & 0.423 \\
Warm Dust Temperature & 0.569 & 0.237 & 0.106 & 0.271 & 0.271 & 0.129 \\
Cold Dust Mass & 0.095 & 0.072 & 0.462 & 0.964 & $\mathbf{0 . 0 2 2}$ & 0.423 \\
Total Warm Dust Mass & 0.682 & 0.678 & $\mathbf{0 . 0 1 9}$ & 0.767 & $\mathbf{0 . 0 2 2}$ & 0.129 \\
Birth Cloud Warm Dust Mass & 0.912 & 0.678 & $\mathbf{0 . 0 1 9}$ & 0.492 & $\mathbf{0 . 0 2 2}$ & 0.129 \\
SFR/Dust Luminosity & 0.238 & 0.224 & 0.999 & 0.492 & 0.271 & 0.883 \\
SFR/Birth Cloud Warm Dust Mass & 0.119 & 0.478 & 0.462 & 0.492 & 0.492 & 0.883 \\
Dust Mass/Stellar Mass & 0.644 & 0.916 & 0.857 & 0.767 & 0.271 & 0.423 \\
Birth Cloud /Cold ISM Dust Mass & 0.719 & 0.224 & 0.037 & 0.767 & 0.271 & 0.129
\end{tabular}

Note. - The probabilities given in this table are the probability that the same population yielded the compared samples, so small values indicate a common population is unlikely. The marginally significant differences are given in bold. The total dust mass is composed of warm dust in the stellar birth clouds and the diffuse ISM and cold dust in the diffuse ISM.

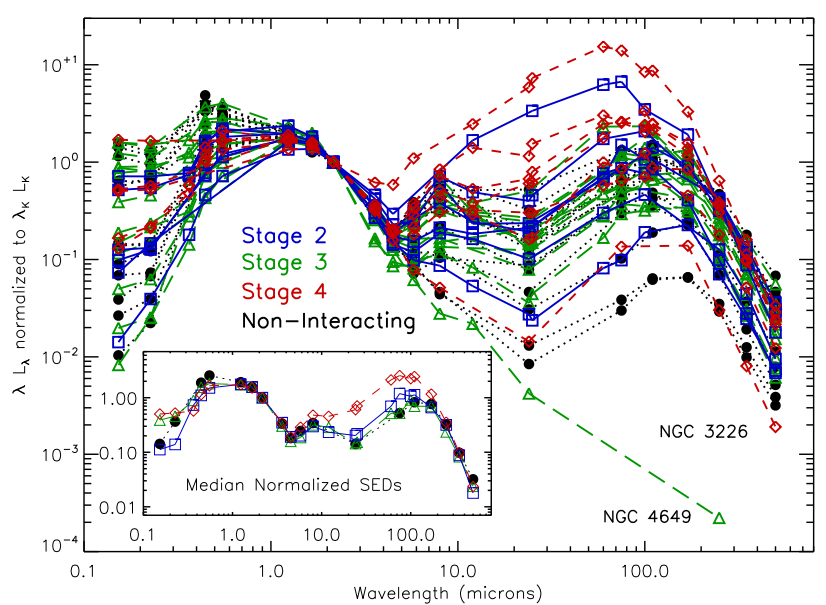

FIG. 17.- SEDs normalized to the 2MASS Ks band luminosity for each galaxy. Stage 2 (weakly interacting), Stage 3 (moderately interacting), Stage 4 (strongly interacting), and non-interacting galaxies are shown respectively in blue squares joined with solid lines, green triangles joined with long dashes, red diamonds joined with short dashes, and black circles joined with dotted lines, respectively. Inset, we show the median SED for each class of galaxies. These SEDs show a tendency for Stage 4 galaxies to have more hot-warm dust emission in the $10-60 \mu \mathrm{m}$ range relative to both its cold dust emission in the SPIRE bandpasses and its stellar NIR emission, whereas the ratio of NIR to FIR emission is relatively consistent. Additionally, the more strongly interacting galaxies typically have a younger stellar population than the Stage 2 galaxies as suggested by the relative amounts of UV to NIR emission. The two labeled galaxies are elliptical galaxies.

with a few caveats. First, with only thirty-one galaxies, our sample has limited statistical power to identify significant variations, especially with half the galaxies in Stage 3. Analysis of trends in SFR and sSFR with the full SIGS sample (Brassington et al. 2013, in preparation), which covers the stages much more uniformly, will have greater statistical power (albeit these comparisons lack the $\mathrm{Her}$ schel SPIRE data and SED analysis that provides more accurate SFR measurements). Second, our classification scheme permits us to examine parameter variations with respect to the strength of the interaction. While this sequence crudely mimics an interaction, the dynamics of two colliding galaxies often includes multiple encounters

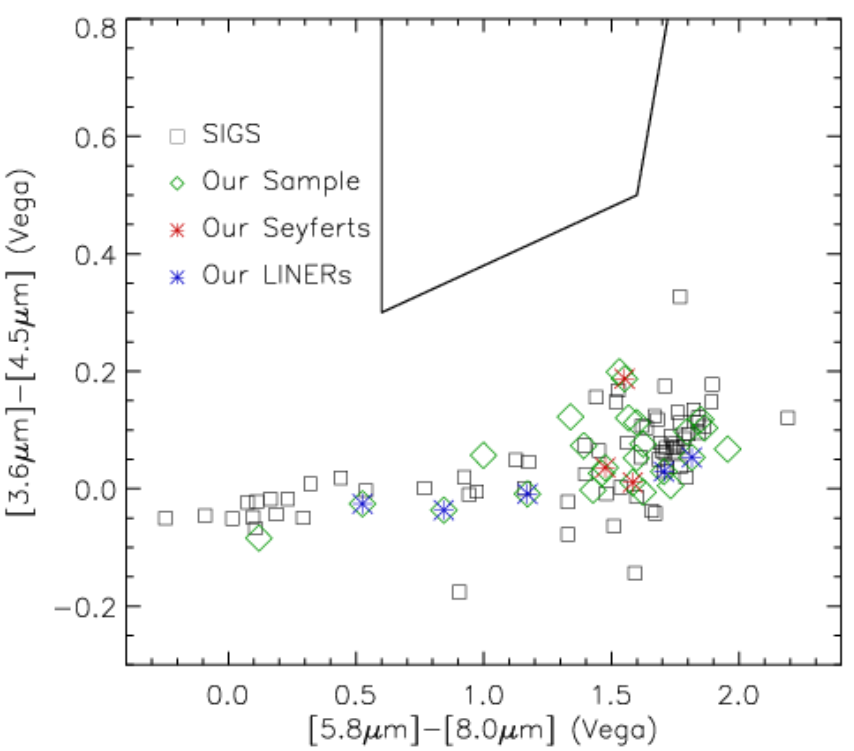

FIG. 18. - IRAC color-color plot of the SIGS galaxies (square) with our sample galaxies (diamonds) over-plotted, showing that none fall in the wedge identified by Stern et al. (2005) as galaxies hosting AGN. Our sample galaxies identified optically as Seyferts or LINERs in Keel et al. (1985) or Ho et al. (1997, 2000) and are marked with red and blue stars, respectively.

prior to final coalescence, modifying the level of star formation at intermediate stages (e.g., Torrey et al. 2012), as well as the intensity of the final burst (e.g., Hopkins et al. 2008, 2009). As a result, interacting systems often do not progress linearly through the interaction stages defined by our classification system.

\subsection{Relative Importance of Specific Data Sets in Constraining Galaxy Parameters}

For each galaxy, we ran six MAGPHYS fits to measure the relative importance of UV, SPIRE, and MIR-FIR data in constraining the value of the derived SFR; stellar mass; sSFR; and dust temperatures, luminosity, and masses. We did this by comparing fits with all available data with fits using a subset of the complete dataset in order to determine if and how the absence of a particular dataset results in a systematic over- or under-estimation 


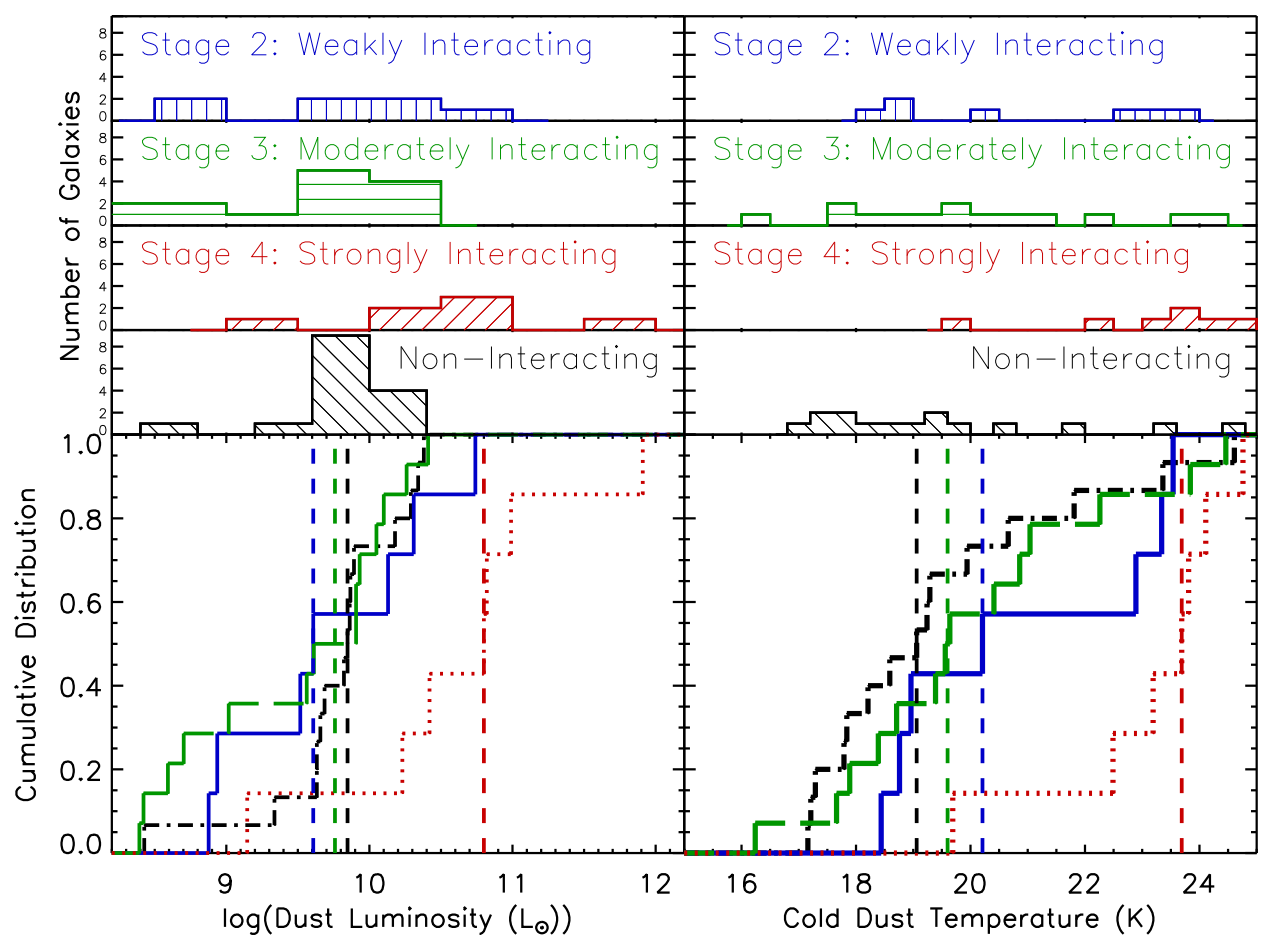

FIG. 19.- Histograms (top) and cumulative distributions (bottom) of the dust luminosity (left) and cold dust temperature (right) derived by MAGPHYS for the three interaction stages defined by the classification system of Dopita et al. (2002), where Stages $2-4$ are weakly (blue, solid), moderately (green, long dashed), and strongly (red, short dashed) interacting, respectively. Non-interacting galaxies are shown in black dot-dashed lines. There is a difference in both luminosity and temperature between the Stages 3 and 4 populations and between Stage 4 and non-interacting populations as defined by a K-S Test (see Table 11). The vertical dotted lines give the median value for each stage. The median dust luminosity is lowest for the 'weakly interacting' Stage 2 galaxies and increases by more than an order of magnitude for the 'strongly interacting' Stage 4 galaxies. The 15-25 K dust temperature is noticeably higher in the Stage 4 galaxies.

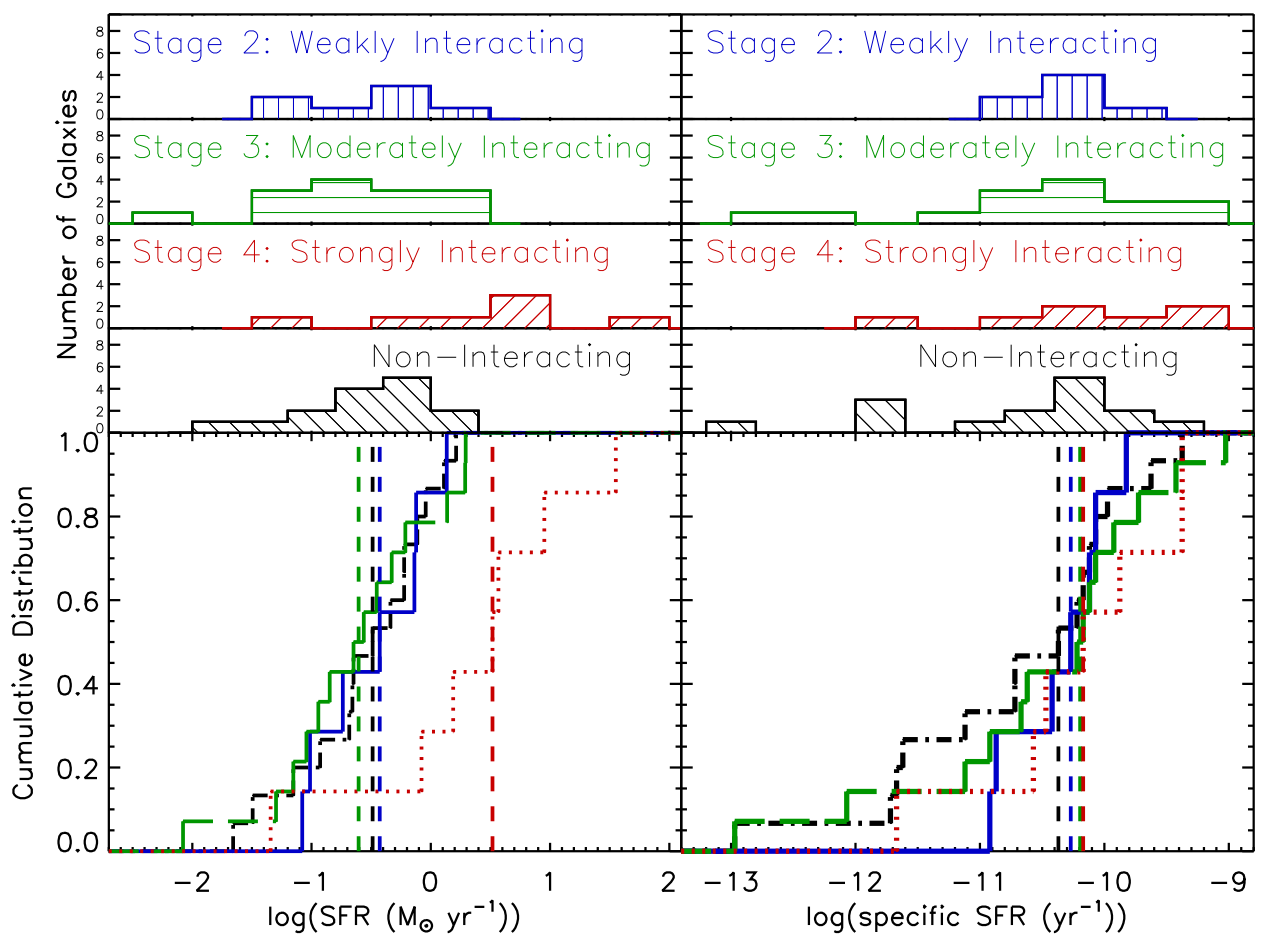

FIG. 20.- Histograms (top) and cumulative distributions (bottom) of SFR (left) and sSFR (right) derived by MAGPHYS for each interaction stage and the control sample shown with the same color and linestyles as Figure 19. SFR shows an increase between noninteracting through moderately interacting galaxies and the Stage 4, 'strongly interacting' systems, an evolution similar to that of dust luminosity. In contrast, the sSFR distributions are very similar, as is supported by the results of the K-S tests. 

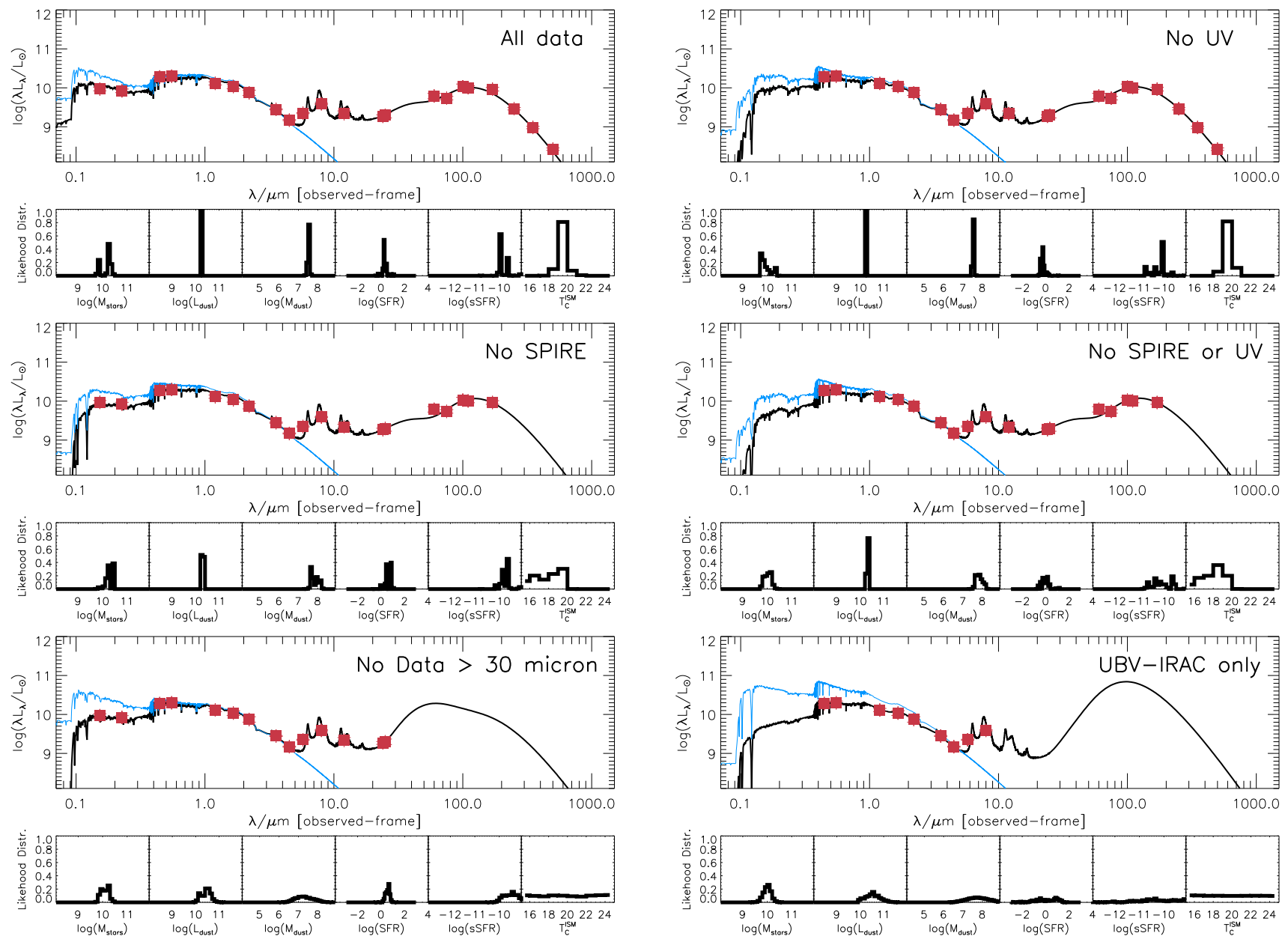

Fig. 21. - MAGPHYS fits with the 6 different data sets for M101, as a representative example of the set of fits done for each galaxy, showing the increasingly constrained parameters (from lower right to top left) as more data are used. UV photometry (present in the left panels) is crucial in constraining sSFR, while SPIRE data (present in the top two panels) is essential for the determination of cold dust temperature and dust mass. The lower right panel demonstrates the limited constraints that ground based photometry alone can provide. 


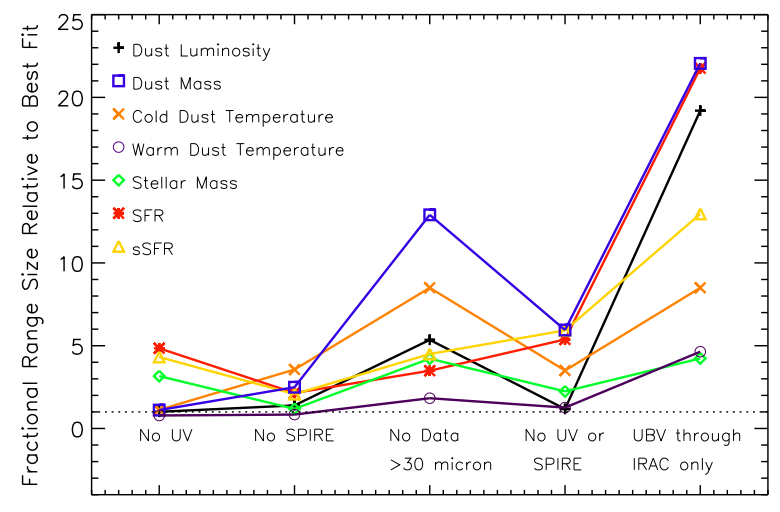

FIG. 22.- Parameter confidence intervals increase with the omission of data. Points indicate the median size of the $68 \%$ confidence interval in the probability distribution function (PDF) for each parameter for the whole set of galaxies relative to the range when all of the data are used in the fit. Using all available data, the median $68 \%$ ranges are: $6.3 \times 10^{8} \mathrm{~L}_{\odot}$ (dust luminosity), $1.8 \times 10^{6} \mathrm{M}_{\odot}$ (dust mass), $0.80 \mathrm{~K}$ (cold dust temperature), $4.4 \mathrm{~K}$ (warm dust temperature), $2.1 \times 10^{9} \mathrm{M}_{\odot}$ (stellar mass), $0.047 \mathrm{M}_{\odot} \mathrm{yr}^{-1}$ (SFR), and $1.3 \times 10^{-11} \mathrm{yr}^{-1}$ (sSFR). UV data are crucial for the determination of sSFR, whereas dust luminosity, mass, and 15-25 K temperature are best constrained by SPIRE data with further constraints applied by photometry from $30-200 \mu \mathrm{m}$.

of these parameters. Figure 21 shows a representative example: the best-fit SEDs for all six fits for M101 as well as the accompanying PDFs for the parameters of interest.

As expected, the SFR is significantly constrained by UV data. This is demonstrated in Figure 22, where the median $68 \%$ confidence interval size is a factor of $\sim 4$ larger in the absence of UV photometry. While stellar mass is fairly well constrained by the UBV-IRAC data alone, the absence of UV data tends to result in younger stellar population templates being selected by MAGPHYS. This effect can be seen in the differences in the UV slope and the strength of the Lyman and Balmer breaks in the various panels of Figure 21. When a younger stellar population template is selected, a smaller fraction of the stellar emission is assumed to originate from late-type stars, resulting in a tendency to estimate the stellar mass $\sim 10-20 \%$ lower than when all the data are used. This can be seen in Figure 23 where we plot the median fractional difference in the value of the galaxy properties for fits with incomplete data sets. UV photometry constrains both the SFR and stellar mass, and it is also the most important wavelength regime to constrain the sSFR. The absence of UV data also tends to result in an over-estimation of the SFR resulting in an estimate of the sSFR $\sim 40 \%$ higher than in fits using all available data.

Herschel data are particularly crucial in constraining the cold dust temperature. PACS data typically outline the peak of the IR emission, but in cases of the coldest dust temperatures, PACS $170 \mu \mathrm{m}$ is typically too indeterminate and it is only in conjunction with the SPIRE $250 \mu \mathrm{m}$ data that the cold dust temperature is reasonably constrained. In contrast, as expected, warm dust temperature is not well constrained by the SPIRE data. Dust luminosity is typically well-estimated with a com-

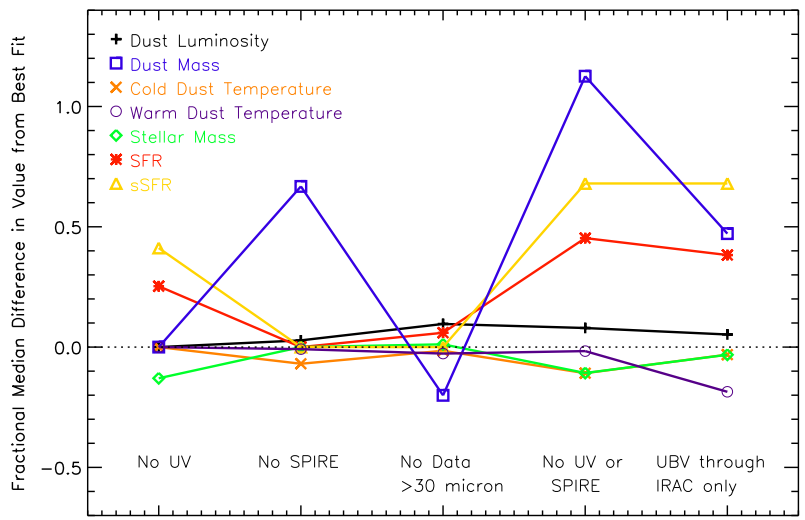

FIG. 23. - Median fractional difference for each parameter relative to its value that parameter when all of the data are used in the fit. The absence of UV data results in sSFR $\sim 40 \%$ higher than in fits using all the data. Dust mass, made up primarily of cold dust, is very sensitive to changes in the cold dust temperature.

bination of IRAS and PACS data, only becoming about a factor of $\sim 2$ more uncertain in absence of SPIRE data.

SPIRE observations are crucial for constraining the dust mass, whose $68 \%$ confidence interval would be at least a factor of 3 larger without SPIRE data. When MIR data at wavelengths $\lambda \geq 30 \mu \mathrm{m}$ are likewise absent, the dust mass becomes almost completely unconstrained as the SED contains little information about the dust emission. The dust mass estimate is the most sensitive to the absence of specific datasets. Interestingly, the dust mass estimated by MAGPHYS is $\sim 60 \%$ higher when SPIRE data are excluded as compared with when all data are used; however, when photometry at wavelengths $\lambda \geq 30 \mu \mathrm{m}$ is excluded, the dust mass is estimated $\sim 20 \%$ lower than when all data are used. The over-estimates in dust mass are correlated with the under-estimates in the cold dust temperature. Since, cold dust tends to make up the bulk of the dust mass and because cold dust mass varies as $T^{-6}$ (assuming it is modeled as a $\beta=2$ modified blackbody), a $5 \%$ change in the dust temperature results in a $30-40 \%$ difference in the dust mass. Aniano et al. (2012) recently showed similar results for NGC 628 and NGC 6946, where fits undertaken only with data at wavelengths $\lambda \leq 170 \mu \mathrm{m}$ tend to overpredict the emission in the SPIRE bands and the associated cold dust mass. When all data at wavelengths $\lambda \geq 30 \mu \mathrm{m}$ are omitted, MAGPHYS relies primarily on more common UV-NIR dominated sources.

\subsection{Comparison of the SFR Derived from the SED to Monochromatic and Broad-band Relations}

Because the SFR is such a key physical parameter, many statistical heuristic attempts have been developed to estimate it from simple observables such as the UV flux (as measured by GALEX) or $\mathrm{H} \alpha$, the $24 \mu \mathrm{m}$ flux, the total IR flux, and combinations of UV and MIR fluxes (e.g., Calzetti 2012). We compared the SFR derived by MAGPHYS to the expected SFR based on a wide range of relations (Figure 24): from the FUV relations of Salim et al. (2007) and Treyer et al. (2010), from the MIPS $24 \mu \mathrm{m}$ emission relations of Rieke et al. (2009) and Zhu et al. (2008, as given in Calzetti et al. 2010), from the 


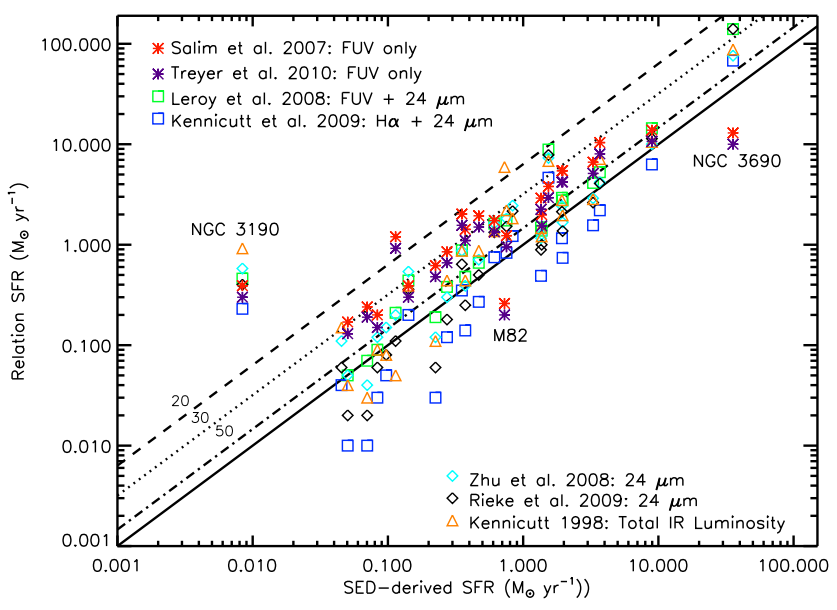

FIG. 24.- Comparison of the SED-derived SFR to those from various SFR proportionality relations from the literature. The solid line shows where the points should lie if the relations agree with the SED-derived values, which is the SFR averaged over $100 \mathrm{Myr}$. The dotted, dashed, and dot-dashed lines shows SFR averaged over 20, 30 , and $50 \mathrm{Myr}$, respectively. SFR is modeled as an exponential burst with a peak value such that the average over $100 \mathrm{Myr}$ is given by the MAGPHYS SFR and decay times of 20, 30, and 50 Myr. While the relations depending solely or partially on IR emission agree well with the MAGPHYS SFR, the FUV relations are typically high and agree better with a SFR modeled as an exponential burst with a width of 30-50 Myr.

relations combining $\mathrm{UV}$ and $24 \mu \mathrm{m}$ emission of Leroy et al. (2008) and Kennicutt et al. (2009), and from the total IR luminosity relation of Kennicutt (1998). We correct the FUV emission from dust using the prescription given in Salim et al. (2007) and use the dust luminosity derived by MAGPHYS for the total IR luminosity. We assume a Chabrier (2003) initial mass function (IMF) and therefore have applied correction factors of 1.06 for relations that assume a Kroupa (2001) IMF and 1.60 for relations that assume a Salpeter (1955) IMF, following Calzetti (2012) and Schiminovich et al. 2007. Figure 24 shows the resulting values plotted against the SFR determined by MAGPHYS.

One trend is quickly apparent: the SFRs estimated from UV photometry alone tend to be high compared with the SED-derived SFR, whereas the relations based solely or partially on IR photometry agree fairly well, at least for SFR greater than $\sim 0.1 \mathrm{M}_{\odot} \mathrm{yr}^{-1}$. Median differences are of $0.7-0.9 \mathrm{M}_{\odot} \mathrm{yr}^{-1}$ for the FUV relations. Interestingly, our most active galaxy, NGC 3690/IC694, shows the inverse trend, as does a prototype starburst M82. This may indicate that the correction for dust is insufficient for these systems. The outlier of NGC 3190 (Fig. 24) is due to the low SFR associated with the best MAGPHYS model for this galaxy that significantly underestimated its UV emission, likely due to the edgeon geometry of the system.

The apparent over-estimation of the SFR by the FUV relations is rooted in the time over which the SFR is estimated in MAGPHYS. FUV emission is dominated by star formation within the past $50 \mathrm{Myr}$, although midto-late B stars can also contribute a significant fraction (Calzetti 2012). In contrast, the IR relations typically assume that a fraction of the stellar light is absorbed to heat dust, and as a result, while the youngest and hottest stars dominate the heating of hot dust, the accumulation of low-mass stars contribute significantly to the heating of the more diffuse dust. Hence, the IR relations represent star formation over a longer timescale. The SFRs derived by MAGPHYS are averaged over the last 100 Myr, which is more consistent with the timescales associated with the IR-dependent SFR relations. Figure 24 also shows the SFRs averaged over shorter time periods with SFR modeled as an exponential decay with a peak value such that the average over $100 \mathrm{Myr}$ is the MAGPHYS SFR value and decay times of 20, 30, and 50 Myr, respectively. The SFRs estimated from the FUV emission agree better with an exponential decay star formation history with a width between 30 and $50 \mathrm{Myr}$, which is the expected timescale of a starburst episode.

\section{SUMMARY}

We modeled the FUV-FIR SEDs of fourteen groups of thirty-one interacting galaxies, typically with $15-25$ flux points, to determine the most probable evolution of dust luminosity, star formation rate, specific star formation rate, dust mass, stellar mass, and dust temperature. The systems were classified as either weakly, moderately, or strongly interacting (Stages 2-4 in the Dopita et al. (2002) scheme). The broad similarities in SED shape between different stages emphasize one key conclusion from this study: as the interaction progresses, and even as bursts of star formation may occur, the changes are most clearly seen not in the distribution of energy broadly but in minor and subtle changes to the SED shapes. Bulk SED properties change little, and only gradually, in typical interactions. Strongly interacting galaxies typically have SEDs characterized by stronger MIR emission relative to both their NIR and FIR emission and more UV emission relative to their NIR emission.

There are marginally statistically differences (as determined by a K-S Test) in the derived galaxy properties: dust luminosity and mass, SFR, and cold dust temperature increase from Stage 3 to Stage 4, SFR increase from Stage 2 to Stage 4, and dust luminosity, SFR, and cold dust temperature increases from the non-interacting galaxies to the Stage 4 galaxies. In contrast, the sSFR does not show variations with interaction stage. The relative constancy of the sSFR between the different stages suggests that this lack of evolution is not due to uncertainty in stage classification or in the association of interaction stage and progress along the interaction. Rather, our set of interacting galaxies shows no clear evidence for a burst of star formation prompted by the interactions or that such effects occur on timescales such that we see enhancements in both stellar mass and SFR, leaving the sSFR relatively stable. This suggests a need to be circumspect about this canonical activity during the early stages of galaxy mergers.

Different wavelengths have different effects in constraining galaxy parameters in the MAGPHYS SED analysis. UV data are important in accurately determining stellar population age and that they are important contributors to determining accurately the (specific) star formation rate. The stellar mass is primarily determined by UBV-IRAC data. SPIRE data are crucial in determining the dust mass; in its absence the cold dust temperature tends to be underestimated, because the location of the peak is much less constrained without data longward of $170 \mu \mathrm{m}$. Cold dust mass, which tends to 
make up the bulk of the dust mass, goes as $T^{-6}$ (assuming $\beta=2$ ), so the change in the dust mass is large for even a small underestimate in the dust temperature. The possible contributions to the SED from AGN are modest for this sample and do not affect our conclusions.

The SFRs derived by MAGPHYS agree reasonably well with simple relations based solely or partially on IR photometry. Relations based on corrected FUV emission tended to overestimate SFR compared to the SEDderived SFR, which is averaged over 100 Myr. The SFR estimated from FUV can best be understood if it represents an exponential decay star formation history with a width of 30-50 Myr.

The complete SIGS sample will bring a significant increase in statistical power in determining galaxy property trends. In addition, testing the accuracy of MAGPHYS against simulations of interacting galaxies will help improve the diagnostic power of SEDs. In a future paper, we will examine what kind of simulated interactions and their parameters best reproduce observed systems and their SEDs. Further, we will test how well MAGPHYS recovers galaxy parameters as a function of their inter- action details.

We are grateful to Gregory Snyder, Maria Koutoulaki, and Flora Stanley for their classifications of our galaxies, Steven Willner for his classifications and helpful comments, Volker Tolls for his assistance in processing Herschel data, Don Neill and Susan Neff at the GALEX helpdesk for the reprocessing of the observation of NGC 3690/IC 694, Erik Hoversten for his assistance with regards to the UVOT corrections, Christopher Klein for his preparation of the IRAC observations, and Joseph Hora for his assistance with galaxies on the edge of SPIRE observations. This work was based on archival data obtained from the Spitzer Science Archive, the Mikulski Archive for Space Telescopes (MAST), the Swift data archive, and the Herschel Science Archive. This research has made use of the NASA/IPAC Extragalactic Database (NED), which is operated by the Jet Propulsion Laboratory, California Institute of Technology, under contract with the National Aeronautics and Space Administration. This work was supported in party NASA grant NNX10AD68G and NASA JPL RSA 1369566. AZ acknowledges support from AR-12011X Chandra grant and EU IRG grant 224878.

\section{APPENDIX}

\section{CLASSIFICATION SCHEME}

The classification methodology used to determine the interaction stage of each system is based on the Dopita et al. (2002) classification scheme. Stage 1 galaxies are non-interacting and Stage 5 galaxies are post-merger or coalescence systems. Stages 2-4 are weakly, moderately, and strongly interacting systems, based on their degree of morphological distortion. In Figure 25, we show a representative system from our sample. The Stage 2 galaxies show little distortion and the galaxies are typically well separated. The Stage 3 galaxies have a range of proximity and show some degree of distortion and the Stage 4 galaxies show significant morphological distortion and are typically close even in projection.

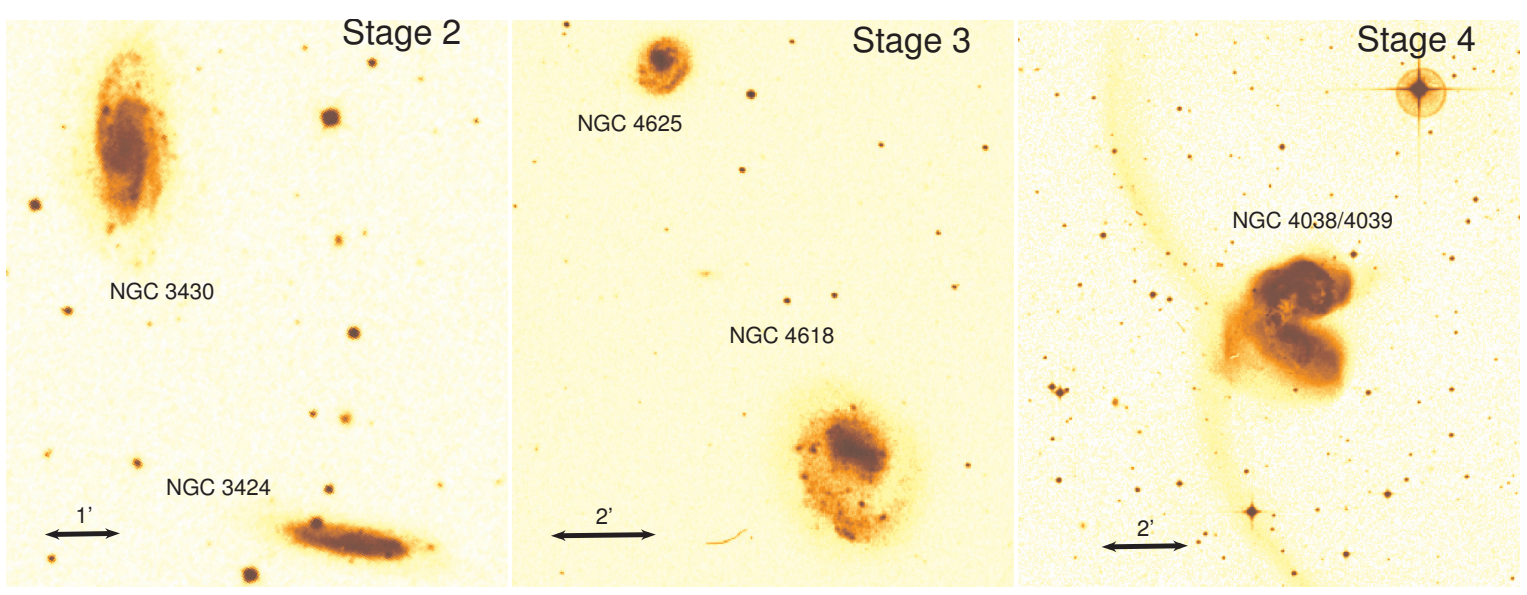

FIG. 25.- Representative examples of Stage 2-4 (left-right) galaxies, showing increasing morphological distortion.

\section{PROCESSING CONCERNS}

\section{PACS and High Pass Filters}

In the course of processing the PACS photometry, we initially used the PhotProject pipeline scripts to make mosaics. When we compared the measured fluxes to values obtained by the MIPS and IRAS instruments at the same wavelengths, we found some were more than a factor of 2 too low, with M101 having the greatest disagreement. This disagreement is the result of the range of angular extents of the galaxies. The PhotProject pipeline includes a high pass filtering algorithm that removes a significant portion of the extended emission in the field and therefore affects nearby galaxies with more extended emission much more egregiously. As a result, we found it essential for nearby 
galaxies to use the Madmap pipeline.

\section{Swift UVOT and Coincidence Losses}

As we noted in $\S 3.3 .2$, the UVOT telescope is vulnerable to coincidence losses. When multiple photons arrive in the same pixel within the same frame, only a single photon is counted. Losses become greater than $1 \%$ when the count rate exceeds 0.007 counts per second per pixel. While corrections for these losses and the uncertainties involved is well determined for point sources (Poole et al. 2008, Kuin \& Rosen 2008), there is a lack of similar understanding for extended sources. Although some of the sample galaxies, notably the starburst galaxies M82 and Arp 299, have count rates high enough over most of their surface that would result in significant coincidence losses, even for the rest of the sample have regions of high fluxes, typically limited to the nuclear area and star forming regions. We therefore opted to analyze only those galaxies lacking GALEX photometry as well as an additional test case, as described in §3.3.2. Fortunately for the two galaxies having UVOT, but lacking $G A L E X$, only the nucleus and a few small regions are bright enough to require corrections. We excluded the regions with high count rates and then measured them independently as point sources, correcting them for coincidence losses using the method of Poole et al. (2008). These corrections always account for less than $1.5 \%$ of the count rate. Based on our test case of NGC 3424, the agreement between the GALEX and UVOT photometry is excellent.

\section{NOTES ON INDIVIDUAL GALAXIES}

Group 1:

NGC 2976: A small galaxy on the outskirts of the M81 group, NGC 2976 has two active, infrared bright regions at the edges of its disk, whose presence is almost completely missed in the UV and NIR.

NGC 3031: Also known as M81, this galaxy is in our closest system and has very clear spiral arms and filamentary structure seen in all the images across our whole filter range. Its nucleus is a LINER. The best-fit decomposition of the IR spectrum performed with DECOMPIR has a $4 \%$ AGN contribution to the total IR emission and a $16 \%$ contribution in the $8-35 \mu \mathrm{m}$ range.

NGC 3034: M82 is one of the nearest starburst galaxies and has a strong galactic outflow perpendicular to its stellar disk, which is visible in both the UV and Herschel images. Its IRAC $5.8 \mu \mathrm{m}$, IRAC $8.0 \mu \mathrm{m}$, and MIPS $24 \mu \mathrm{m}$ images are saturated. Its SED is among the worst fit by MAGPHYS with the current set of models. This may be due in part to the high obscuration - its fit has a $\left\langle\tau_{V}\right\rangle=2.9$ - or perhaps to the inclusion of some emission from the outflow, which MAGPHYS does not model. We measured the contribution of the outflow above and below the disk of the galaxy within the aperture and found that it contributes $\sim 20-30 \%$ of the UV emission and $\sim 10-20 \%$ of the emission at wavelengths greater than $150 \mu \mathrm{m}$, which does not fully account for the discrepancy in the UV bands. We selected the $3.6 \mu \mathrm{m}$-derived aperture to minimize the contribution of the emission from the outflow. The outflow's UV emission is primarily scattered light from the disk, while its IR emission is from the dust in the outflow. Since MAGPHYS cannot model such a feature, its inclusion would tend to bias the best-fit model.

NGC 3077: Another small galaxy in the M81 group, NGC 3077 lies behind a nearby bright star that prevented it from being observed in the UV by GALEX and UVOT. Its MIR-FIR images shows evidence of tidal stripping in the asymmetric structure and southwest lobe.

Group 2:

NGC 3185: This Seyfert galaxy shows a circum-galactic ring of star-forming material in both UV and IR images. The DECOMPIR decomposition requires a $3 \%$ and a $12 \%$ contribution of the AGN to the total IR luminosity and 8-35 $\mu \mathrm{m}$ luminosity respectively. Its disk shows up in the FIR image as two bright regions at the opposite ends of the galaxy. It is the most distant member of the triplet it forms with NGC 3190 and NGC 3187 on the sky, but it and NGC 3190 have much closer recessional velocities than NGC $3187\left(1217 \mathrm{~km} \mathrm{~s}^{-1}\right.$ and $1271 \mathrm{~km} \mathrm{~s}^{-1}$ vs $1581 \mathrm{~km}$ $\mathrm{s}^{-1}$ ). Group 2 is the only compact group in our sample, although the SIGS sample has several others. Tzanavaris et al. (2010) found a bi-modality for the sSFR distributions of compact groups depending on the slope of the IRAC photometry between $4.5 \mu$ mand $8.0 \mu \mathrm{m}$. NGC 3185 has a negative IRAC slope and its sSFR places it in the relatively quiescent population as expected by Tzanavaris et al. (2010).

NGC 3187: This galaxy has a pair of tidally elongated arms, which are best detected in the UV. This galaxy did not have archival IRAS fluxes. NGC 3187 has a positive IRAC slope and its sSFR places on the edge of the distribution of galaxies with positive slopes, argued by Tzanavaris et al. (2010) to indicate active star formation.

NGC 3190: The most massive of the three galaxies in this group, NGC 3190 is a nearly edge on LINER and has a dust lane, that appears prominently in absorption in UV and is correspondingly bright in IR emission. Its SED shows particularly low UV relative to its visible emission, but presumably the geometry of this dust lane explains the poor fit to the UV and the disagreement in the estimates of its SFR. The DECOMPIR best-fit requires no contribution from an AGN. NGC 3190 has a negative IRAC slope, but the low SFR value derived by MAGPHYS places it in the quiescent category of Tzanavaris et al. (2010).

Group 3:

NGC 3226: As an elliptical galaxy, NGC 3226's emission is dominated by its stars, although there appears to be a faint tidal feature in the $8 \mu \mathrm{m}$ emission directed roughly to the north. NGC 3226 is particularly faint in the MIR relative to its NIR emission and only has upper limits in the MIR-FIR from IRAS. It has a LINER nucleus, but the decomposition of its IR spectrum indicates no significant contribution form an AGN.

NGC 3227: Along with its smaller companion NGC 3226, NGC 3227 was not observed by GALEX, so we use UVOT 
data instead. Its IRS spectrum shows [Ne V] emission consistent with a Seyfert nucleus. Its DECOMPIR fits are the worst of the nine galaxies, but have the highest AGN contribution of $15-25 \%$ to the total IR luminosity and $45-60 \%$ of the MIR luminosity.

Group 4:

NGC 3395/3396: The apertures of this pair of galaxies overlapped sufficiently to make determination of the emission belonging to each galaxy problematic. We therefore opted to treat the system as a combined system. This pair of galaxies is distantly associated with Group 5. This group does not have UBV photometry. The SED shows little attenuation in the UV and strong $60 \mu \mathrm{m}$ emission relative to the $100 \mu \mathrm{m}$ emission.

Group 5:

NGC 3424: This edge-on galaxy's central region becomes increasingly bright relative to its disk with increasing wavelength. Similarly to NGC 3190, the other nearly-edge on galaxy, its NUV-FUV slope is quite steep and not particularly well fit by MAGPHYS. We used this galaxy as a test case for analyzing UVOT photometry and found good agreement with the GALEX photometry. It lacks UBV photometry.

NGC 3430: Seen nearly face-on, this galaxy has a large and extended UV disk. This system provides a nice example of a system fairly early in the interaction sequence and hence fairly undisturbed morphologically. The IR peak of its SED is not very well constrained as this galaxy was not observed by PACS and did not have MIPS $70 \mu \mathrm{m}$ and $160 \mu \mathrm{m}$ fluxes available.

Group 6:

NGC 3448: This is the larger member of this pair of dwarf galaxies. A bridge of emission extends from NGC 3448 in the direction of UGC 6016, seen most prominently in the NUV.

UGC 6016: This dwarf galaxy is very faint in the IR, but has a large but diffuse UV envelope. Due to its lack of significant detections in several of our filters and in ancillary IRAS, we cannot constrain the galaxy parameters very tightly. Its distance is also the most uncertain of the sample as it did not have a distance modulus or a recessional velocity in the $\mathrm{PSCz}$ catalog.

Group 7:

NGC 3690/IC 694: Also known as Arp 299, this system is the most active in our sample, with the highest amount of star formation and is our only LIRG, showing a corresponding large amount of UV attenuation. Its $8 \mu \mathrm{m}$ image is saturated, and it does not have ancillary UBV fluxes.

Group 8:

NGC 3786/88: The galaxies in this pair have very similar UV-NIR fluxes, but NGC 3788, which is more edge-on, has higher fluxes in the Herschel bands. However, their best-fit SEDs have similar infrared luminosities, likely due to the relatively high contribution of the warm dust in the model for NGC 3786 compared to NGC 3788. Neither galaxy has ancillary IRAS or UBV fluxes. NGC 3786 is a Seyfert galaxy, with a bright nuclear region showing [Ne v] emission in its IRS spectrum and a partial ring of star-forming regions. Its decomposition requires an AGN contribution of $7 \%$ and $26 \%$ to the total IR luminosity and MIR luminosity respectively.

Group 9:

NGC 4038/4039: Also known as the Antennae, this pair of galaxies is one of our most evolved systems and, because we cannot separate them, we treat it as a single entity. Its clumpy distribution of star forming regions are clearly apparent at $8 \mu \mathrm{m}$ and in the UV bands; the two nuclei are most clearly seen in 2MASS and IRAC, a reflection of the relative $\mathrm{PAH}$ strengths to warm dust.

Group 10:

NGC 4618: Paired with NGC 4625 in a roughly equal-mass dwarf galaxy interaction, NGC 4618 has an off-center nucleus with a single arm curving to the south-west, features seen in all the images.

NGC 4625: While relatively compact in the infrared, NGC 4625 has a faint, diffuse set of flocculent set of spiral arms observed best in the NUV band.

Group 11:

NGC 4647: This spiral galaxy is located at the edge of the IRAC field, which complicated the measurement of its flux because its aperture, determined on the NUV image, extends past the edge of the IRAC image. We had to manually correct for the edge pixels without flux. We also do not have ancillary UBV fluxes.

NGC 4649: A large elliptical galaxy also known as M60, NGC 4649 is very faint in the mid-infrared and absent in the far-infrared, a dramatic contract to its companion. It has the lowest sSFR of the sample.

Group 12:

M51A: The Whirlpool Galaxy is the larger galaxy in this well-studied system. It has quite consistent morphology across the wavelengths, but with more inter-arm filamentary emission and greater extent in the UV than in the IR. The decomposition of its IR spectrum is best fit without an AGN contribution, despite its LINER nucleus. This is one of the systems where the flux from the smaller galaxy was subtracted from the aperture of the large galaxy.

M51B: The smaller companion to the Whirlpool Galaxy, M51B is dominated by early-type stars and has very little UV emission. Its FIR emission is confined to its nucleus. Its MIPS $160 \mu \mathrm{m}$ measurement was a factor of three lower than the PACS measurement, and we opted to omit it from the fits.

Group 13:

NGC 5394/95: The smaller galaxy of our most distant pair, NGC 5394 shows a beautiful pair of tidal tails, especially in the UV. NGC 5395 has a LINER nucleus, which requires only a small AGN contributions of $3 \%$ and $12 \%$ to the galaxy's total IR and MIR luminosities respectively in the DECOMPIR fits. This is the other system where the small galaxy flux needed to be subtracted from the large galaxy aperture. 
Group 14:

M101: Another well-studied galaxy, its GALEX, Spitzer, and Herschel images show clumpy star forming region structures along the spiral arms. Its large size made determining a single unbroken aperture with SExtractor complicated. As a result, some of the outermost UV emitting regions were not included.

NGC 5474: A small companion to M101, its core is offset to the north from its center.

\section{REFERENCES}

Aniano, G. et al., 2012, ApJ, 756, 138

Barnes, J. E., 1992, ApJ, 393, 484

Barnes, J. E. \& Hernquist, L. 1996, ApJ, 495, 187

Bertin, E. \& Arnouts, S. 1996, A\&AS, 117, 393

Blain, A., Jameson, A., Smail, I., Longhair, M., Kneib, J., \& Invison, R. 1999, MNRAS, 309, 715

Breeveld, A. A., et al. 2010, MNRAS, 406, 1687

Bruzual, G. \& Charlot, S. 2003, MNRAS, 344, 1000

Bruzual, A. G., 2007, in IAU Symp. Vol. 241, On TP-AGB Stars and the Mass of Galaxies, ed. A. Vazdekis. \& R. F. Peletier (Cambridge: Cambridge Univ. Press), 125

Calzetti, D. 2012, arXiv:1208.2997

Calzetti, D. et al. 2010, ApJ, 714, 1256

Casasola, V., Bettoni, D., \& Galletta, G. 2004, å, 422, 941

Chabrier, G. 2003, PASP, 115, 763

Charlot, S. \& Fall, S. M., 2000, ApJ, 539, 718

Cohen, M., Megeath, S. T., Hammersley, P. L., Martín-Luis, F., \& Stauffer, J. 2003, AJ, 125, 2645

da Cunha, E., Charlot, S., \& Elbaz, D. 2008, MNRAS, 388, 1597

Dale, D. A. et al. 2005, ApJ, 633, 857

Dale, D. A. et al. 2007, ApJ, 655, 863

Dale, D. A. et al. 2009, ApJ, 703, 517

Dale, D. A. et al. 2012, ApJ, 745, 95

Dahari, O. 1985, ApJS, 57, 643

de Vaucouleurs, G., et al. 1991, Third Reference Catalogue of Bright Galaxies, (New York: Springer)

Di Matteo, T., Springel, V., \& Hernquist, L. 2005, Nature, 433, 604

Di Matteo, T., Combes, F., Melchior, A. L., \& Semelin, B. 2007, A\&A, 468, 61

D’Onghia, E., Vogelsberger, M., Faucher-Giguere, C.-A., \& Hernquist, L. 2010, ApJ, 725, 353

Dopita, M. A., Pereira, M., Kewley, L. J., \& Capaccioli, M. 2002, ApJS, 143, 47

Dubinski, J., Mihos, J. C., \& Hernquist, L. 1996, ApJ, 462, 576

Dubinski, J., Mihos, J. C., \& Hernquist, L. 1999, ApJ, 526, 607

Elbaz, D. et al. 2011, A\&A 533, A119

Ellison, S. L., Patton, D. R., Mendel, J. T., \& Scudder, J. M. 2011, MNRAS, 418, 2043

Engelbrach, C. W., et al. 2007, PASP, 119, 994

Engelbracht, C. W., Rieke, G. H., Gordon, K. D., Smith, J.-D., Werner, M. W., Moustakas, J., Willmer, C. N. A., \& Vanzi, L. 2008, ApJ, 678, 804

Fazio, G. G. et al. 2004, ApJS, 154, 10

Freedman, W. L., et al. 2001, ApJ, 553, 47

Gehrels, N. et al. 2004, ApJ, 611, 1005

Goddard Space Flight Center. 2004, GALEX Observer's Guide (Greenbelt, MD:GSFC) http://galexgi.gsfc.nasa.gov/ docs/galex/Documents/GALEXObserverGuide.pdf

Griffin, M. J., et al. 2010, A\&A, 518, L3

Hayward, C. C., Kereš, D., Jonsson, P., Narayanan, D., Cox, T. J., \& Hernquist, L. 2011, ApJ, 743, 159

Hayward, C. C., Jonsson, P., Kereš, D., Magnelli, B., Hernquist, L., \& Cox, T. J. 2012a, MNRAS, 424, 951

Hayward, C. C., Narayanan, D., Kereš, D., Jonsson, P., Hopkins, P. F., Cox, T. J., \& Hernquist, L. 2012b, arXiv:1209.2413

Ho, L. C., Filippenko, A. V., \& Sargent, W. L. W. 1997, ApJS, 112,315

Ho, L. C., et al. 2000, ApJ, 541, 120

Hoversten, E. A. et al. 2011, AJ, 141, 205

Hopkins, P. F., Hernquist, L., Cox, T. J., Di Matteo, T., Robertson, B., \& Springel, V. 2006, ApJS, 163, 1

Hopkins, P. F., Hernquist, L., Cox, T. J., Dutta, S. N., \& Rothberg, B. 2008, ApJ, 679, 156

Hopkins, P. F., Cox, T. J., Dutta, S. N., Hernquist, L., Kormnedy, K., \& Lauer, T. R. 2009, ApJS, 181, 135

Hopkins, P. F. 2012, MNRAS, 420, L8
Jarrett, T. H., Chester, T., Cutri, R., Schneider, S. E., \& Huchra, J. P. 2003, AJ, 125, 525

Jonsson, P. 2006, MNRAS, 2006, 372, 2

Joye, W. A. \& Mandel, E. 2003, in ASP. Conf. Ser. 295 Astronomical Data Analysis Softward and Systems XII, ed. H. E. Payne, R. I. Jedrzejewski, \& R. N. Hooks (San Francisco: ASP)

Karachentsev, I. D., Kudrya, Y. N., Karachentseva, V. E., \& Mitronova, S. N., 2006, Astrophysics, 49, 450

Keel, W. C., Kennicutt Jr., R. C., Hummel, E., \& Van der Hulst, J. M. 1985, AJ, 90, 708

Kennicutt, R. 1998, ARA\&A, 46, 189

Kennicutt, R. et al. 2003, PASP, 115, 928

Kennicutt, R. et al. 2009, ApJ, 703, 1672

Kennicutt, R. et al. 2011, PASP, 123, 1347

Kewley, L. J., Heisler, C. A., Dopita, M. A., \& Lumsden, S. 2001, ApJS, 132, 37

Kocevski, D. et al. 2012, ApJ, 744, 148

Kroupa, P. 2001, MNRAS, 322, 231

Kuin, N. P. M. \& Rosen, S. R. 2008, MNRAS, 383, 383

Lambas, D. G., Tissera, P. B., Alonso, M. S., \& Coldwell, G. 2003, MNRAS, 346, 1189

Leroy, A. K., Walter, F., Brinks, E., Bigiel, F., De Blok,

W. J., G., Madore, B., \& Thornley, M. D., 2008, ApJ, 136, 2782

Li, C. et al. 2008, MNRAS, 385, 1915

Lisenfeld, U. et al., 2007, A\&A, 462, 507

Madden S. C., Galliano F., Jones A. P., \& Sauvage M., 2006, A\&A, 446, 877

Makovoz, D. \& Khan I., 2005, in ASP Conf. Ser. 132,

Astronomical Data Analysis Software and Systems VI, ed. P. L.

Shopbell, M. C. Britton, \& R. Ebert (San Francisco: ASP)

Martin, D. et al. 2005, ApJ, 619, L1

Mihos, J. C. \& Hernquist, L. 1994, ApJ, 431, L9

Mihos, J. C. \& Hernquist, L. 1996, ApJ, 464, 641

Moshir, M. et al. 1990, Infrared Astronomical Satellite Catalogs,

The Faint Source Catalog, version 2.0

Mould, J. R. et al. 2000, ApJ, 529, 786

Mullaney, J. R., Alexander, D. M., Goulding, A. D., \& Hickox, R. C. 2011, MNRAS, 414, 1082

Narayanan, D., Hayward, C. C., Cox, T. J., Hernquist, L., Jonsson, P., Younger, J. D., \& Groves, B. 2010a, MNRAS, 401, 1613

Narayanan, D. et al. 2010b, MNRAS, 407, 1701

Ott, S. 2010, in ASP Conf. Ser. 434, Astronomical Data Analysis Software and Systems XIX, ed. Y. Mizumoto, K.-I. Morita, \& M. Ohishi (San Francisco: ASP)

Paladini, R., Linz, H., Altieri, B., \& Ali, B. 2012, Assessment analysis of the extended emission calibration for the PACS red channel, Document: PICC-NHSC-TR-034 (Pasadena, CA: NHSC)

Pilbratt, G. L., et al., 2010, A\&A, 518, L1

Poglitsch, A. et al. 2010, A\&A, 518, L2

Poole, T. S., et al. 2008, MNRAS, 383, 627

Press, W. H., Teukolsky, S. A., Vetterling, W. T., \& Flannery, B. P. 2007, Numerical Recipes: The Art of Scientific Computing, 3rd ed. (New York: Cambridge Univ. Press)

Rice, W., Lonsdale, C. J., Soifer, B. T., Neugebauer, G., Kopan, E. L., Lloyd, L. A., De Jong, T., \& Habing, H. J. 1988, ApJS, 68,91

Rich, J. A., Torrey, P., Kewley, L. J., Dopita, M. A., \& Rupke, D. S. N. 2012, ApJ, 753, 5

Rieke, G. et al. 2004, ApJS, 154, 25

Rieke, G. et al. 2009, ApJ, 692, 556

Salim, S. et al. 2007, ApJ, 173, 267

Salpeter, E. E. 1955, ApJ, 121, 161

Sanders, D. 1999, Ap\&SS, 266, 331

Sanders, D. B., Soifer, B. T., Elias, J. H., Neugebauer, G., \& Matthews, K. 1988, ApJ, 328, L35 
Sanders, D. B., Mazzarella, J. M., Kim, D.-C., Surace, J. A., \& Soifer, B. T. 2003, AJ, 126, 1607

Saunders, W. et al. 2000, MNRAS, 317, 55

Schiminovic, D. et al. 2007, ApJ, 173, 315

Schuster, M. T., Marengo, M. \& Patten, B. M. 2006, SPIE, 6270, 65

Scudder, J. M., Ellison, S. L., Torrey, P., Patton, D. R., \& Mendel, J. T. 2012, arXiv:1207.4791

Silverman, J. D. et al. 2011, ApJ, 743, 2

Skrutskie, M. F. et al. 2006, AJ, 131,1163

Smith, B. J., Struck, C., Hancock, M., Appleton, P. N. Charmandaris, V., \& Reach, W. T. 2007, ApJ, 133, 719

Soifer, B. T., Boehmer, G., Neugebauer, G., Sanders, D. B. 1989, AJ, 98, 766

Springel, V. 2005, MNRAS, 364, 1105

Springel, V. , Di Matteo, T., \& Hernquist, L. 2005, MNRAS,361, 776

Stern, D. et al. 2005, ApJ, 631, 163

Surace, J. A., Sanders, D. B., \& Mazzarella, J. M. 2004, AJ, 127, 3235

Swinyard, B. M. et al. 2010, A\&A, 518, L4

Tonry, J. L., Dressler, A., Blakeslee, J. P., Ajhar, E. A., Fletcher, A. B., Luppino, G. A., Metzger, M. R., \& Moore, C. B. 2001, ApJ, 546, 681
Toomre, A. \& Toomre, J. 1972, ApJ, 178, 623

Torrey, P., Cox, T. J., Kewley, L., \& Hernquist, L. 2012, ApJ, 746,108

Treyer, M. et al. 2010, ApJ, 719, 1191

Tully, R. B. \& Fisher, J. R., 1977, A\&A, 54, 661

Tully, R. B., Shaya, E. J., Karachentsev, I. D., Courtois, H. M., Kocevski, D. D., Rizzi, L., \& Peel, A. 2008, ApJ, 676, 184

Tully, R. B. 1994, VizieR Online Data Catalog, 7145, 0

Veilleux, S., Kim, D. C., \& Sanders, D. B. 2002, ApJS, 143, 315

Werner, M. W. et al. 2004, ApJS, 154, 1

Wyder, T. K. et al. 2005, ApJ, 619, L15

$\mathrm{Xu}, \mathrm{C} . \mathrm{K}$. et al. 2010, ApJ, 713, 330

Yuan, F.-T., Takeuchi, T. T., Matsuoka, Y., Buat, V., Burgarella, D., \& Iglesias-Páramo, å, 548, 117

Younger, J. D., Hayward, C. C., Narayanan, D., Cox, T. J. Hernquist, L., \& Jonsson, P. 2009, MNRAS, 396, L66

Zibetti, S., Gallazzi, A., Charlot, S., Pierini, D., \& Pasquali, A. 2012, arXiv:1205.4717

Zhu, Y.-N., Wu, H., Cao, C., \& Li, H.-N. 2008, ApJ, 686, 155 